\title{
ON DYNAMIC FEEDBACK LINEARIZATION OF FOUR-DIMENSIONAL AFFINE CONTROL SYSTEMS WITH TWO INPUTS
}

\author{
JEAN-BAPTISTE POMET
}

\begin{abstract}
This paper considers control affine systems in $\mathbb{R}^{4}$ with two inputs, and gives necessary and sufficient conditions for dynamic feedback linearization of these systems with the restriction that the "linearizing outputs" must be some functions of the original state and inputs only. This also gives conditions for non-affine systems in $\mathbb{R}^{3}$.
\end{abstract}

\section{INTRODUCTION}

A deterministic finite dimensional nonlinear control system

$$
\dot{x}=f(x, u)
$$

where the state $x$ lives in $\mathbb{R}^{n}$, the control $u$ lives in $\mathbb{R}^{m}$, and $f$ is smooth - smooth means $\mathcal{C}^{\infty}$ in this article - is said to be locally static feedback equivalent around $(\bar{x}, \bar{u})$ to another system

$$
\dot{z}=\tilde{f}(z, v)
$$

around $(\bar{z}, \bar{v})$ if there exists a nonsingular feedback transformation, i.e. two maps

$$
\begin{aligned}
& u=\alpha(x, v) \\
& x=\phi(z)
\end{aligned}
$$

such that $(z, v) \mapsto(\phi(z), \alpha(z, v))$ is a local diffeomorphism sending $(\bar{z}, \bar{v})$ to $(\bar{x}, \bar{u})$, that transforms (1.1) into (1.2). The interest of feedback equivalence is that the transformation (1.3) allows one to convert the solution to a certain control problem for system (1.1) to the solution of a similar control problem for system (1.2). It is clear that (germs of) static feedback transformations form a group acting on (germs of) systems, and that static feedback equivalence is an equivalence relation. This feedback equivalence has been very much studied, see for instance $[4,3,13]$. Classification of control systems modulo this equivalence is of course a very ambitious and difficult program, almost out of reach. A more restricted problem is the one of describing the orbits of controllable linear systems, i.e. systems of the form $\dot{z}=A z+B v$ with (controllability) the columns of $B, A B, A^{2} B, A^{3} B, \ldots$

I.N.R.I.A., 2004 route des lucioles, BP. 93, 06902 Sophia Antipolis, France.

E-mail: pometesophia.inria.fr.

This work was initiated when the author was with: Laboratoire d'Automatique de Nantes, U.R.A. C.N.R.S. 823, E.C.N., Univ. de Nantes, 1 rue de la Noë, 44072 NANTES cédex 03, France.

Received by the journal March 26, 1996. Revised December 7, 1996. Accepted for publication February 19, 1997.

(c) Société de Mathématiques Appliquées et Industrielles. Typeset by $\mathrm{AT}_{\mathrm{E} X} \mathrm{X}$. 
having full rank. This problem is known as static feedback linearization, and has been completely solved: in [15, 12], explicit conditions are given for a general nonlinear system to be locally static feedback equivalent to a controllable linear system.

A dynamic feedback, or dynamic compensator, as opposed to static, is one where the "old" controls $u$ are not computed from the "new" ones $v$ by simply static functions (1.3), but through a dynamic system which has a certain state $\xi$ :

$$
\begin{aligned}
u & =\alpha(x, \xi, v) \\
\dot{\xi} & =\gamma(x, \xi, v) \\
z & =\phi(x, \xi),
\end{aligned}
$$

where $\xi$ lives in $\mathbb{R}^{\ell}, \ell>0$, and $\phi$ is a (local) diffeomorphism of $\mathbb{R}^{n+\ell}$. $(x, v)$ may be viewed as the "input" of the control system, and $(u, x, \xi)$, or $(u, X)$ as its "output".

Clearly, (1.4) allows one to transform system (1.1) into a system like (1.2). However, contrary to the case of static feedback, the dimension of the state of the transformed system (1.2) is strictly larger than the dimension of the state of the original system (1.1), and for this reason, it a priori difficult to say what an "invertible" dynamic feedback "transformation" can be. One may however, following [7], state the problem of dynamic feedback linearization as the one of deciding when a system (1.1) can be transformed via a dynamic feedback (1.4) into a linear controllable system. The problem of deciding if a given system is dynamic feedback linearizable is much more difficult than the one for static feedback and is still open. A panorama and further references on dynamic feedback linearization from the point of view of compensators (1.4) can be found in [7]. This reference contains some sufficient conditions, that have the annoying drawback of not being invariant by static feedback, and also the following three results, that are of more general interest: a single input system $(u \in \mathbb{R})$, at a "regular" point, is dynamic feedback linearizable if and only if it is static feedback linearizable; dynamic feedback linearizability at a rest point $(x, u)=(\bar{x}, 0)$ implies controllability of the linear approximation of the system at this point; a controllable system which is affine in the control -i.e. the right-hand side of (1.1) is affine with respect to $u$ - and such that the dimension of the state is larger than the dimension of the control by at most one is always dynamic feedback linearizable.

As seen above, the case of systems with one control is completely understood outside singularities, so that the nontrivial cases have at least two controls. The cases where the dimension of the state is less than 3 are somehow trivial (again, away from singularities), and the case where it is 3 and the system is affine in the control is covered by the above mentioned result from [7]. The smallest nontrivial cases are therefore non-affine systems with three states and two inputs, and affine systems with four states and two inputs. Section 6 explains how to apply the results of this paper to three-dimensional non-affine systems, but the rest of the paper is devoted to systems

$$
\dot{x}=X_{0}(x)+u_{1} X_{1}(x)+u_{2} X_{2}(x)
$$


where $x \in \mathbb{R}^{4}$ and $u_{1}$ and $u_{2}$ are in $\mathbb{R}\left(u=\left(u_{1}, u_{2}\right)\right) . X_{0}, X_{1}$ and $X_{2}$ are smooth vector fields in $\mathbb{R}^{4}$. Smooth means $\mathcal{C}^{\infty}$ in this article.

Of course, since it is the simplest non-trivial case, the problem of dynamic feedback linearization for the four dimensional system (1.5) has already been studied. In [17], based on the results from [7], sufficient conditions on $X_{0}, X_{1}$ and $X_{2}$ are given. A drawback of these results is that they are not invariant by static feedback, and are only sufficient conditions. They are contained in the results of the present paper.

Rather recently, some conceptual advances have been made on dynamic equivalence and dynamic linearization, initiated in $[18,8]$ (see [11] for a complete exposition). In [18], a restricted class of compensators (1.4) is studied, called endogenous dynamic feedbacks. They are exactly these that should be called "invertible". They are the compensators (1.4) such that, by differentiating relations (1.1) and (1.4), it is possible to express $\xi$ and $v$ as functions of $x, u, \dot{u}$, and a finite number of time-derivatives of $u$. The compensator (1.4) may then be replaced by some formulas giving $z$ and $v$ as functions of $(x, u, \dot{u}, \ddot{u}, \ldots)$, which is "invertible" by formulas giving $x$ and $u$ as functions of $(z, v, \dot{v}, \ddot{v}, \ldots)$. On the other hand, the notion of differential flatness for control systems is introduced in [18, 8, 11], as roughly speaking, existence of $m$ - two for system (1.5) - functions of $x, u, \dot{u}$ and a finite number of time-derivatives of $u$ which are differentially independent (the Jacobian of any finite number of these functions and their time derivatives has maximum rank) and such that both $x$ and $u$ can be expressed as functions of these $m$ functions and a finite number of their time-derivatives. These functions are called linearizing outputs, or "flat outputs". It is proved there that differential flatness is equivalent to equivalence by endogenous $\mathrm{dy}$ namic feedback to a controllable linear system. In the differential algebraic framework of $[8,11]$, flatness is defined as the differential field representing the system being non-differentially algebraic over a purely transcendental differential extension of the base field, and the linearizing output is a transcendence basis. Of course, the linearizing outputs are then "restricted" to be algebraic. With a suitable definition of endogenous dynamic equivalence between differential fields, it is proved that differential flatness is equivalent to equivalence by endogenous dynamic feedback to a controllable linear system.

In [14], a notion of dynamic equivalence in terms of transformations on solutions of the system is studied; different types of transformations are defined there in terms of infinite jets of trajectories, for smooth systems, one of them is proved there to be exactly the one studied here. A property of "freedom" is introduced that is close to differential flatness and is proved to be equivalent to equivalence to a linear system.

See [11], [7] or [2, 1] for a more complete panorama and list of references on dynamic feedback equivalence and dynamic feedback linearization, with references to recent and interesting results and points of views that we do not discuss here, like the work by Shadwick [23] (and subsequent articles) that make a link between dynamic feedback linearization and the notion of absolute equivalence defined by E. Cartan for Pfaffian systems.

There was a need to develop a geometric framework for the invertible transformations that represent dynamic feedback. This was done by the 
author in [19] and independently by the authors of $[8,11]$ in $[9,10]$. In these papers, an (infinite dimensional) differential geometric approach, based on infinite jet spaces, is used, and the transformations described above may be seen as diffeomorphism that conjugate a system to another, they are a particular case of infinite order contact transformations, or Lie-Bäcklund transformations used in the "geometric" study of differential systems and partial differential relations.

Here, we adopt the notations and the precise definitions for linearizing outputs and dynamic linearization from $[19,1]$. They are summed up in section 2.2 and 2.3 .

The problem of deciding endogenous ${ }^{1}$ dynamic linearizability is then the one of deciding existence of a system of linearizing outputs. The first difficulty is that there is no known a priori bound on the number of timederivatives of the input the linearizing outputs should depend upon (similarly, there is no a priori bound on the dimension of $\xi$ in a compensator (1.4) that would transform a given nonlinear system into a linear system if such a compensator exists). Even for four-dimensional systems (1.5), no such bound is known. We do not address this difficulty in the present paper. We only give necessary and sufficient conditions for existence of linearizing outputs depending on $x$ and $u$. We call $x$-dynamic and $(x, u)$-dynamic linearizability existence of linearizing outputs depending on $u$ or on $(x, u)$. Note that the present conditions are quite explicit: a small package in Maple, described in [16], that helps in the process of checking the present conditions, will soon be available from the author.

Technically, the results in this paper amount to conditions for existence of solutions to some differential relations: in principle, given a system, one may write the PDEs that a pair of functions $\left(h_{1}(x, u), h_{2}(x, u)\right)$ has to satisfy to be a pair of linearizing outputs, and then check whether this system of PDEs has some solutions (formal integrability, Spencer co-homology, see for instance [6] or [21]). This program reaches its limits very quickly seen the complexity of the PDEs themselves, and of the computation of compatibility conditions: even if algorithms are theoretically available, writing the PDEs for linearizing outputs for a general system is already heavy, and computing the compatibility conditions via general algorithms is overwhelming. The essence of the paper is however to compute these compatibility conditions, but in a way that uses a lot of the structure of the problem and makes them tractable. In particular, we use, for the case of linearizing outputs depending on $x$ and $u$, the "infinitesimal Brunovsky form" introduced in [2, 1, 20], that allows to write different PDEs: the unknowns are then some coefficients of transformations that act on pairs of differential forms - the condition is that it makes them integrable- instead of the linearizing outputs themselves. It would be interesting to know whether it is general that the use of the infinitesimal Brunovský form provides a method to write the equations for linearizing outputs in a more tractable manner. This is explained into details in section 2, see especially subsection 2.6 for a discussion of the two possible

\footnotetext{
${ }^{1}$ It is announced in $[11,10]$ that general dynamic feedback linearizability implies endogenous dynamic linearizability. From such a result, existence of linearizing outputs would be necessary and sufficient for general dynamic feedback linearization.

Esaim: CoCV, June 1997, VOL. 2, Pf. 151-230
} 
ways of writing the equations for existence of linearizing outputs, either directly or via the infinitesimal Brunovsky form.

The paper is organized as follows. Section 2 recalls or introduces some technical material, including the precise definitions of what is intended here by feedback linearization and linearizing outputs in the geometric context of $[19,1]$. Sections 3 and 4 contain the results, i.e. necessary and sufficient conditions for $x$-dynamic linearization (section 3 ) and for $(x, u)$-dynamic linearization (section 4 ). Section 6 shows that non affine systems in $\mathbb{R}^{3}$ which are dynamic feedback linearizable may be transformed into an affine system (1.5) in $\mathbb{R}^{4}$ by a simple dynamic extension, using a result by Rouchon [22] or Sluis [24]. Most proofs are in section 7, and some basic facts on Pfaffian systems used in them are recalled in the Appendix. Section 8 makes some remarks on the problems we leave open and on the interest and limitations of the techniques we use.

\section{Statement of the Problem}

\subsection{Static Feedback}

A static feedback transformation, around a point $(\bar{x}, \bar{u})$ is a local transformation on the controls $v=\phi_{2}(x, u)$, defined on a neighborhood of $(\bar{x}, \bar{u})$, with $\frac{\partial \phi_{2}}{\partial u}$ invertible (the reason for the subscript " 2 " is that we shall use a local diffeomorphism $\phi_{1}$ on $x$, so that $(x, u) \mapsto\left(\phi_{1}(x), \phi_{2}(x, u)\right.$ is a local diffeomorphism on $(x, u))$.

Since we are only concerned with systems like (1.5) where the controls appear linearly, we shall only need affine static feedback. A local affine static feedback transformation is one of the above type where $\phi_{2}$ is affine with respect to $u$. It is more convenient to write the inverse of $\phi_{2}$ with respect to $u$, i.e. to write, instead of $\left(v_{1}, v_{2}\right)=\phi_{2}\left(x, u_{1}, u_{2}\right)$,

$$
\left(\begin{array}{l}
u_{1} \\
u_{2}
\end{array}\right)=\alpha(x)\left(\begin{array}{l}
v_{1} \\
v_{2}
\end{array}\right)+\beta(x)
$$

with $\alpha(x)$ an invertible $2 \times 2$ matrix and $\beta(x)$ a vector, both depending smoothly on $x$. It transforms system (1.5) into

$$
\begin{aligned}
& \dot{x}=\tilde{X}_{0}(x)+v_{1} \widetilde{X}_{1}(x)+v_{2} \widetilde{X}_{2}(x) \\
& \text { with }\left\{\begin{array}{l}
\widetilde{\widetilde{X}}_{0}=X_{0}+\beta_{1} X_{1}+\beta_{2} X_{2} \\
\widetilde{X}_{1}=\alpha_{11} X_{1}+\alpha_{21} X_{2} \\
\widetilde{X}_{2}=\alpha_{12} X_{1}+\alpha_{22} X_{2} .
\end{array}\right.
\end{aligned}
$$

A system is locally static feedback linearizable if and only if it may be transformed by such a transformation into a system which, in some coordinates $z=\phi_{1}(x)$, reads like a controllable linear system $\dot{z}=A z+B v$ in $\mathbb{R}^{4}$ with two inputs; these linear systems are all of the form (a) or (b) below, up to a linear feedback - like (2.1) with $\alpha$ and $\beta$ constant - and a linear change of coordinates:

$$
\text { (a) }\left\{\begin{array} { l } 
{ \dot { z } _ { 1 } = z _ { 2 } } \\
{ \dot { z } _ { 2 } = z _ { 3 } } \\
{ \dot { z } _ { 3 } = u _ { 1 } } \\
{ \dot { z } _ { 4 } = u _ { 2 } }
\end{array} \quad \text { (b) } \left\{\begin{array}{l}
\dot{z}_{1}=z_{2} \\
\dot{z}_{2}=u_{1} \\
\dot{z}_{3}=z_{4} \\
\dot{z}_{4}=u_{2}
\end{array}\right.\right.
$$


These are the two Brunovský canonical forms for controllable linear systems with two inputs and four states, see [5]. Static feedback linearizable systems are a particular case of $x$-dynamic linearizable systems because $\left(x_{1}, x_{4}\right)$ for the form (a), and $\left(x_{1}, x_{3}\right)$ for the form (b) may be chosen as a pair of linearizing outputs (see section 2.3).

Static feedback will also be used in the present paper to give some simple "normal" forms modulo this transformation and a change of coordinates on $x$ of the systems considered for each case, or set of conditions, see (3.3), (3.4), $(3.6),(3.7),(3.9),(3.20),(4.13),(4.19)$. The term feedback invariant refers to a property or an object that is invariant with respect to this equivalence relation between systems.

\section{2. "Infinite dimensional" Differential CalCulus and EQUIVALENCE BY ENDOGENOUS FEEDBACK}

This section is devoted to briefly recalling some notations and results from $[19,1]$. The reader is referred to these references for a detailed exposition.

As mentioned in the introduction, similar material was also presented independently - in $[9,10]$. The content of $[9,10]$ is more general and more formal, and tends to give as a conclusion that systems (1.1) is not a general enough class of system for control theory, whereas [19] aims at developing the sufficient framework to use classical tools from differential calculus for the study of dynamic feedback. This infinite dimensional framework is, in any case, a rather convenient way of manipulating functions and other objects which depend on a finite but not a priori fixed number of variables, and it allows to say that the transformations by dynamic feedback are "diffeomorphisms".

We call generalized state manifold for system (1.1) with $n$ states and $m$ inputs the "infinite dimensional manifold" $\mathcal{M}_{\infty}^{m, n}$ where a set of coordinates is $\left(x_{1}, \ldots, x_{n}, u_{1}, \ldots, u_{m}, \dot{u}_{1}, \ldots, \dot{u}_{m}, \ddot{u}_{1}, \ldots, \ddot{u}_{m}, \ldots\right)$. It is the projective limit of the finite dimensional manifolds $\mathcal{M}_{K}^{m, n}, K \geq-1$ with coordinates $\left(x_{1}, \ldots, x_{n}, u_{1}, \ldots, u_{m}, \dot{u}_{1}, \ldots, \dot{u}_{m}, \ldots, u_{1}^{(K)}, \ldots, u_{m}^{(K)}\right)$-when $K=-1$, this means $\left(x_{1}, \ldots, x_{n}\right)$ - and we have the obvious projections $\pi_{K}$ from $\mathcal{M}_{\infty}^{m, n}$ to $\mathcal{M}_{K}^{m, n}:$

$$
\pi_{K}\left(x_{1} \ldots x_{n}, u_{1} \ldots u_{m}, \ldots\right)=\left(x_{1} \ldots x_{n}, u_{1} \ldots u_{m}, \ldots u_{1}^{(K)} \ldots u_{m}^{(K)}\right) \text {. }
$$

The topology is the product topology, the least fine such that all these projections are continuous, i.e. an open set is always of the form $\pi_{K}^{-1}(O)$ with $O$ a (finite-dimensional) open subset of $\mathcal{M}_{K}^{m, n}$. In particular when a property holds locally around a point $\left(x, u, \dot{u}, \ddot{u}, u^{(3)}, \ldots\right)$, it means that it holds on a neighborhood of this point, i.e. for points whose first coordinates (an unknown a priori but finite number) are close to these of the original point, but with no restriction on the remaining coordinates. Actually, we will often say "in a neighborhood of $\left(x, u, \ldots, u^{(K)}\right)$ " to indicate that the value of $\left(u^{(K+1)}, u^{(K+2)}, \ldots\right)$ does not matter, i.e. the neighborhood is of the form $\pi_{K}^{-1}(O)$ with $O$ a neighborhood of $\left(x, u, \ldots, u^{(K)}\right)$ in $\mathcal{M}_{K}^{m, n}$.

Smooth functions are functions of a finite number of coordinates which are smooth in the usual sense. Differential forms of degree 1 are finite linear combinations: 
$a_{-1}^{1} \mathrm{~d} x_{1}+\cdots+a_{-1}^{n} \mathrm{~d} x_{n}+a_{0}^{1} \mathrm{~d} u_{1}+\cdots+a_{0}^{m} \mathrm{~d} u_{m}+\cdots+a_{J}^{1} \mathrm{~d} u_{1}^{(J)}+\cdots+a_{J}^{m} \mathrm{~d} u_{m}^{(J)}$ where the $a_{i}^{j}$ s s are smooth function. Forms of any degree may be defined similarly. Vector fields are (possibly infinite) linear combinations $b_{-1}^{1} \frac{\partial}{\partial x_{1}}+$ $\cdots+b_{-1}^{n} \frac{\partial}{\partial x_{n}}+b_{0}^{1} \frac{\partial}{\partial u_{1}}+\cdots+b_{0}^{m} \frac{\partial}{\partial u_{m}}+b_{1}^{1} \frac{\partial}{\partial \dot{u}_{1}}+\cdots+b_{1}^{m} \frac{\partial}{\partial \dot{u}_{m}}+\cdots$. Note that this infinite sum is only symbolic. There is no notion of "convergence" here since a vector field may be defined as a derivation on smooth functions, which, by definition depend only on a finite number of variables, so that the sum becomes finite when computing the Lie derivative of a smooth function along this vector field.

A diffeomorphism is a mapping $\varphi$ from $\mathcal{M}_{\infty}^{m, n}$ to $\mathcal{M}_{\infty}^{\tilde{m}, \tilde{n}}$ which is invertible and such that $\varphi$ and $\varphi^{-1}$ are smooth mappings, in the sense that, for any smooth function $h$ from $\mathcal{M}_{\infty}^{\tilde{m}, \tilde{n}}$ to $\mathbb{R}, h \circ \varphi$ is a smooth function from $\mathcal{M}_{\infty}^{m, n}$ to $\mathbb{R}$, and for any smooth function $k$ from $\mathcal{M}_{\infty}^{m, n}$ to $\mathbb{R}, k \circ \varphi^{-1}$ is a smooth function from $\mathcal{M}_{\infty}^{\tilde{m}, \tilde{n}}$ to $\mathbb{R}$.

A system $\dot{x}=f(x, u)$ with $x \in \mathbb{R}^{n}$ and $u \in \mathbb{R}^{m}$ is represented by a vector field of the form $F=f(x, u) \frac{\partial}{\partial x}+\dot{u}_{1} \frac{\partial}{\partial u_{1}}+\dot{u}_{2} \frac{\partial}{\partial u_{2}}+\ddot{u}_{1} \frac{\partial}{\partial \dot{u}_{1}}+\cdots$ on the manifold $\mathcal{M}_{\infty}^{m, n}$. It is said to be (locally) equivalent by endogenous dynamic feedback to the system $\dot{z}=\tilde{f}(z, v)$ with $z \in \mathbb{R}^{\tilde{n}}$ and $v \in \mathbb{R}^{\tilde{m}}$, itself represented by the vector field $\widetilde{F}=\tilde{f}(z, v) \frac{\partial}{\partial z}+\dot{v}_{1} \frac{\partial}{\partial v_{1}}+\dot{v}_{2} \frac{\partial}{\partial v_{2}}+\ddot{v}_{1} \frac{\partial}{\partial \dot{v}_{1}}+\ldots$ on $\mathcal{M}_{\infty}^{\tilde{m}, \tilde{n}}$ if and only if there exists a (local) diffeomorphism from $\mathcal{M}_{\infty}^{m, n}$ to $\mathcal{M}_{\infty}^{\tilde{m}, \tilde{n}}$ that conjugates these two vector fields. This implies that $\tilde{m}=m$.

These diffeomorphism exactly mimic the transformations defined in [18]. The definition of "endogenous" as opposed to "exogenous" is explained there, or in [11].

From now on, let us focus on the small dimensional system (1.5), i.e. $n=4$ and $m=2$. We associate to system (1.5) the following vector field on $\mathcal{M}_{\infty}^{2,4}$

$$
F=X_{0}+u_{1} X_{1}+u_{2} X_{2}+\dot{u}_{1} \frac{\partial}{\partial u_{1}}+\dot{u}_{2} \frac{\partial}{\partial u_{2}}+\ddot{u}_{1} \frac{\partial}{\partial \dot{u}_{1}}+\cdots
$$

Let us call canonical linear system with two inputs the vector field

$$
C=\dot{v}_{1} \frac{\partial}{\partial v_{1}}+\dot{v}_{2} \frac{\partial}{\partial v_{2}}+\ddot{v}_{1} \frac{\partial}{\partial \dot{v}_{1}}+\ddot{v}_{2} \frac{\partial}{\partial \dot{v}_{2}}+v_{1}^{(3)} \frac{\partial}{\partial \ddot{v}_{1}}+\cdots
$$

on the manifold $\mathcal{M}_{\infty}^{2,0}$ where a set of coordinates is $v_{1}, v_{2}, \dot{v}_{1}, \dot{v}_{2}, \ddot{v}_{1}, \ddot{v}_{2}, \ldots$ Any controllable linear system with 2 inputs can be (globally) transformed via a diffeomorphism into the canonical linear system on $\mathcal{M}_{\infty}^{2,0}$, see [1]. For instance, for the first case in (2.3), the diffeomorphism is given by $v_{1}=$ $x_{1}, \dot{v}_{1}=x_{2}, \ddot{v}_{1}=x_{3}, v_{1}^{(3)}=u_{1}, v_{1}^{(4)}=\dot{u}_{1}, \ldots, v_{2}=x_{4}, \dot{v}_{2}=u_{2}, \ddot{v}_{2}=$ $\dot{u}_{2}, \ldots$. Hence, system (1.5) is said to be locally linearizable by endogenous dynamic feedback, or simply endogenous dynamic linearizable at $\mathcal{X} \in \mathcal{M}_{\infty}^{2,4}$ if and only if there is a diffeomorphism $\varphi$ from an open neighborhood of $\mathcal{X}$ in $\mathcal{M}_{\infty}^{2,4}$ to an open set of $\mathcal{M}_{\infty}^{2,0}$ which transforms the vector field $F$ defined in (2.5) into the vector field $C$ on $\mathcal{M}_{\infty}^{2,0}$.

Let us discuss a few more objects that will be used in the paper. Lie Brackets, exterior derivative, Lie derivatives and all objects from usual differential calculus may be defined because they (or each of their components) 
may all be computed finitely and depend on a finite number of variables; all identities from differential calculus are valid (any given such identity really involves only a finite number of variable).

We call time-derivative along system (1.5) the Lie derivative along the vector field $F$. It corresponds to the derivation defined in the differential fields in [11]. It will often be denoted $\frac{\mathrm{d}}{\mathrm{dt}}$ instead of $L_{F}$. It may be applied to functions: for a function $h\left(x, u, \dot{u}, \ldots, u^{(K)}\right), \dot{h}$, or $L_{F} h$, or $\frac{\mathrm{d}}{\mathrm{dt}} h$, is the function of $x, u, \dot{u}, \ldots, u^{(K+1)}$ obtained by applying the chain rule and substituting $X_{0}(x)+u_{1} X_{1}(x)+u_{2} X_{2}(x)$ for $\dot{x}$. This time-derivative may also be applied to forms. The time-derivative of $\omega=a_{-1}^{1} \mathrm{~d} x_{1}+\cdots+a_{-1}^{4} \mathrm{~d} x_{4}+$ $a_{0}^{1} \mathrm{~d} u_{1}+a_{0}^{2} \mathrm{~d} u_{2}+\cdots+a_{J}^{1} \mathrm{~d} u_{1}^{(J)}+a_{J}^{2} \mathrm{~d} u_{2}^{(J)}$, i.e. its Lie derivative with along $F$, is given by

$$
\begin{gathered}
\dot{\omega}=a_{-1}^{1} \mathrm{~d} \dot{x}_{1}+\cdots+a_{-1}^{4} \mathrm{~d} \dot{x}_{4}+\dot{a}_{-1}^{1} \mathrm{~d} x_{1}+\cdots+\dot{a}_{-1}^{4} \mathrm{~d} x_{4} \\
+a_{0}^{1} \mathrm{~d} \dot{u}_{1}+a_{0}^{2} \mathrm{~d} \dot{u}_{2}+\dot{a}_{0}^{1} \mathrm{~d} u_{1}+\dot{a}_{0}^{2} \mathrm{~d} u_{2}+\cdots \\
\cdots+a_{J}^{1} \mathrm{~d} u_{1}^{(J+1)}+a_{J}^{2} \mathrm{~d} u_{2}^{(J+1)} \dot{a}_{J}^{1} \mathrm{~d} u_{1}^{(J)}+\dot{a}_{J}^{2} \mathrm{~d} u_{2}^{(J)}
\end{gathered}
$$

where $\mathrm{d} \dot{x}_{i}$ stands for the differential of the $i$ th component of $X_{0}+u_{1} X_{1}+$ $u_{2} X_{2}$.

Let us mention one last notation. By $\operatorname{Span}\{d \mathbf{x}\}$ or $\operatorname{Span}\{\mathrm{dx}, \mathrm{d} \mathbf{u}\}$ we mean the module over smooth functions spanned by $\mathrm{d} x_{1}, \mathrm{~d} x_{2}, \mathrm{~d} x_{3}, \mathrm{~d} x_{4}$, or by $\mathrm{d} x_{1}$, $\mathrm{d} x_{2}, \mathrm{~d} x_{3}, \mathrm{~d} x_{4}, \mathrm{~d} u_{1}, \mathrm{~d} u_{2}$ respectively.

\subsection{Linearizing OUtPuts}

Linearizing outputs, or flat outputs were introduced by Fliess, Lévine, Martin and Rouchon in their work on differential flatness. Originally, it was a way to view the problem of dynamic feedback linearization in a more tractable way, but the systems for which there exists linearizing - or flatoutputs, i.e. differentially flat systems, possess properties that are very interesting independently from the fact that they may be rendered linear in some coordinates after adding to them a dynamic compensator: all their solutions may be parameterized "freely" by the linearizing outputs, see [11].

The following is the definition of linearizing output in the framework exposed above. It totally agrees with the one in $[18,11]$.

Definition 2.1 ([1]). A pair of functions $\left(h_{1}, h_{2}\right)$ on $\mathcal{M}_{\infty}^{2,4}$ is called a pair of linearizing outputs on an open subset $U$ of $\mathcal{M}_{\infty}^{2,4}$ if the functions $L_{F}^{j} h_{k}, k \in$ $\{1,2\}, j \geq 0$, are a set of coordinates on $U$, i.e. if $\mathcal{X} \mapsto\left(L_{F}^{j} h_{k}(\mathcal{X})\right)_{k \in\{1,2\}, j \geq 0}$ is a diffeomorphism from $U$ to an open subset of $\mathbb{R}^{2 \mathbb{N}}=\mathcal{M}_{\infty}^{2,0}$.

It is said to be a pair of linearizing output at point $\left(\bar{x}, \bar{u}, \dot{\bar{u}}, \ldots, \bar{u}^{(J)}\right)$ with $J \geq-1$ (when $J=-1$, this stands for $\bar{x}$ ) if it is a pair of linearizing output on an open set $U$ of the form $\pi_{J}^{-1}\left(U_{J}\right)$ (see (2.4)) where $U_{J}$ is a neighborhood of $\left(\bar{x}, \bar{u}, \dot{\bar{u}}, \ldots, \bar{u}^{(J)}\right)$ in $\mathcal{M}_{J}^{2,4}$, i.e. $\mathbb{R}^{2 J+6}$.

The following equivalent formulation is maybe simpler. It is closer to the definition in $[18,11]$.

Proposition 2.2 ([19]). A pair of functions $\left(h_{1}, h_{2}\right)$ on $\mathcal{M}_{\infty}^{2,4}$ is a pair of linearizing outputs at point $\left(\bar{x}, \bar{u}, \dot{\bar{u}}, \ldots, \bar{u}^{(J)}\right)$ with $J \geq-1$ (when $J=-1$, this stands for $\bar{x}$ ) if and only if there exists on open set $U$ of the form $\pi_{J}^{-1}\left(U_{J}\right)$ (see (2.4)) such that

Esaim: Cocv, June 1997, Vol. 2, Pp. 151-230 
1. the differential forms $\left(\mathrm{d} h_{k}^{(j)}\right)_{k \in\{1,2\}, j \geq 0}$ are linearly independent at all points of $U$ (meaning that whenever you take a finite number among these, they are linearly independent),

2. there exists an integer $L$ and a smooth function $\psi$ from an open set of $\mathbb{R}^{2 L+2}$ to $\mathbb{R}^{6}$ such that $(x, u)=\psi\left(h_{1}, h_{2}, \dot{h}_{1}, \dot{h}_{2}, \ldots, h_{1}^{(L)}, h_{2}^{(L)}\right)$ on $U$ (this is an identity between functions of $x, u, \dot{u}, \ddot{u}, \ldots$ ).

As said above, the linearizing outputs have a lot of interest in themselves, when they exist. They are also very relevant for the problem of dynamic linearization, thanks to the following equivalence, pointed out in $[18,8,11]$. Proposition 2.3. (Local) endogenous dynamic linearizability is equivalent to existence (locally) of a pair of linearizing outputs.

A proof in the present context may be found in [19, Theorem 4]. The following may illuminate the above introduced notions.

Proof. A diffeomorphism $\varphi$ that conjugates the vector field $F$ defined in (2.5) to the canonical vector field $C$ on $\mathcal{M}_{\infty}^{2,0}$ defines two functions $h_{1}=v_{1} \circ \varphi$ and $h_{2}=v_{2} \circ \varphi$ on $\mathcal{M}_{\infty}^{2,4}$ which have the property that all their Lie derivatives $L_{F}^{j} h_{k}$ are transformed by the diffeomorphism into the coordinate $v_{k}^{(j)}$, which implies that the functions $L_{F}^{j} h_{k}$ are locally a set of coordinates on $\mathcal{M}_{\infty}^{2,4}$; conversely, if two functions exists which have this property, it is very easy to build a diffeomorphism from $\mathcal{M}_{\infty}^{2,4}$ to $\mathcal{M}_{\infty}^{2,0}$ which transforms $F$ into $C$.

By definition of what a smooth function is, the functions in a pair of linearizing outputs depend only on a finite number of variables among $x, u$, $\dot{u}, \ddot{u} \ldots$ In [1], we say that a system is $\left(x, u, \ldots, u^{(K)}\right)$-dynamic linearizable when there exists a pair of functions depending only on $x, u, \ldots, u^{(K)}$. Clearly, from proposition 2.3 and above, linearizability by endogenous $\mathrm{dy}$ namic feedback implies $\left(x, u, \ldots, u^{(K)}\right)$-dynamic linearizability for a certain $K$. Of course, a very interesting question is: given a system, how to determine a bound $K$ such that if it is dynamic linearizable at all, then it is $\left(x, u, \dot{u}, \ddot{u}, \ldots, u^{(K)}\right)$-dynamic linearizable ? Even for systems of the form (1.5), this is the subject of ongoing research.

As explained in the introduction, we only deal, in the present paper, with linearizing outputs depending on $x$ only, or on $x$ and $u$ :

Definition 2.4. System (1.5) is said to be $(x, u)$-dynamically linearizable at the point $\overline{\mathcal{X}}=\left(\bar{x}, \bar{u}, \ldots, \bar{u}^{(J)}\right)$ if and only if there exists a pair of linearizing outputs $\left(h_{1}, h_{2}\right)$ that depend on $x$ and $u$ only on an open set $\pi_{K}^{-1}(\overline{\mathcal{X}})$, a pair of linearizing outputs depending on $x$ and $u$ only. It is said to be $x$-dynamically linearizable if these linearizing outputs depend on $x$ only.

The present paper characterizes $x$-dynamic linearizability and $(x, u)$-dynamic linearizability for systems (1.5). Systems that are proved here not to be $(x, u)$-dynamic linearizable might or might not be $(x, u, u)$-dynamic linearizable, or $(x, u, \dot{u}, \ddot{u})$-dynamic linearizable, and so on $\ldots$

\subsection{Non-ACCESSIBILITY}

Since we only work at regular points, non-accessibility always means in the present paper (and with the dimensions as in (1.5)) that there exists 
one function $\chi(x)$, or two functions $\chi_{1}(x)$ and $\chi_{2}(x)$, such that $\dot{\chi}=\varphi(\chi)$ for some function $\varphi$, or $\dot{\chi}_{i}=\varphi_{i}\left(\chi_{1}, \chi_{2}\right), i=1,2$ for some functions $\varphi_{1}$ and $\varphi_{2}$.

This is an obstruction to existence of a pair of linearizing outputs. Indeed, if $\left(h_{1}, h_{2}\right)$ is a pair of linearizing outputs, $\dot{\chi}=\varphi(\chi)$, or $\dot{\chi}_{i}=\varphi_{i}\left(\chi_{1}, \chi_{2}\right)$ implies a nontrivial relation between $h_{1}, h_{2}, \dot{h}_{1}, \dot{h}_{2}, \ldots, h_{1}^{(J)}, h_{2}^{(J)}$ for a certain $J>0$, which cannot occur from the definition of a pair of linea rizing outputs.

\subsection{Linearizing Pfaffian systems, infinitesimal Brunovský form}

An infinite set of differential forms is a basis of the space of all differential forms in the neighborhood of a point if any finite number of them are linearly independent at this point and there exists a neighborhood $U$ of this point such that any differential form defined on $U$ may be written as a linear combination of a finite number of the forms in the "basis" with coefficients smooth functions defined in $U$.

Definition 2.5. Let $\omega_{1}$ and $\omega_{2}$ be two differential forms. We say that $\left\{\omega_{1}, \omega_{2}\right\}$ is a linearizing Pfaffian system at a certain point $\left(\bar{x}, \bar{u}, \dot{\bar{u}}, \ldots, \bar{u}^{(J)}\right)$ if and only if $\omega_{1}, \omega_{2}$ and all their time-derivatives, i.e. $\left(\omega_{k}^{(j)}\right)_{k \in\{1,2\}, j \geq 0}$ form a basis of the space of all differential forms in a neighborhood of this point.

Note that this is a property of the Pfaffian system (or the co-distribution) $\left\{\omega_{1}, \omega_{2}\right\}$ rather than the pair of forms since this property will still hold if $\omega_{1}$ and $\omega_{2}$ are replaced by another basis for the same Pfaffian system.

Clearly, if $\left(h_{1}, h_{2}\right)$ is a pair of linearizing outputs, then $\left\{\mathrm{d} h_{1}, \mathrm{~d} h_{2}\right\}$ is a linearizing Pfaffian system because the function $\psi$ in proposition 2.2 translates into a linear combination when differentiating. The converse is also true but requires a local inverse theorem in the "infinite dimensional" framework described above, that is given in [19]:

Proposition 2.6 ([19]). A pair of functions $\left(h_{1}, h_{2}\right)$ is a pair of linearizing outputs at a point if and only if $\left\{\mathrm{d} h_{1}, \mathrm{~d} h_{2}\right\}$ is a linearizing Pfaffian system at this point.

Since we pointed out that being a linearizing Pfaffian system does not depend on the precise choice of the basis, from Frobenius theorem, it is enough to have a linearizing Pfaffian system satisfying Frobenius condition.

Proposition 2.7 ([19]). There exists a pair of linearizing outputs around a point if and only if there exists a linearizing Pfaffian system $\left\{\omega_{1}, \omega_{2}\right\}$ on a neighborhood of this point satisfying Frobenius condition: $\mathrm{d} \omega_{1} \wedge \omega_{1} \wedge \omega_{2}=$ $\mathrm{d} \omega_{2} \wedge \omega_{1} \wedge \omega_{2}=0$ in a neighborhood of this point.

We have the following — straightforward - property that describes all the possible linearizing Pfaffian systems from one:

Proposition $2.8([1])$. Let $\left\{\omega_{1}, \omega_{2}\right\}$ be a linearizing Pfaffian system at a certain point. Then for two forms $\eta_{1}$ and $\eta_{2},\left\{\eta_{1}, \eta_{2}\right\}$ is a linearizing Pfaffian system if and only if $\omega_{1}$ and $\omega_{2}$ are linear combinations of $\eta_{1}, \eta_{2}$ and a finite number of their time derivatives on a neighborhood of this point.

Analogously, a pair of functions $\left(h_{1}, h_{2}\right)$ is a pair of linearizing outputs at this point if and only if $\omega_{1}$ and $\omega_{2}$ are linear combinations of $\mathrm{d} h_{1}, \mathrm{~d} h_{2}$ and a finite number of their time derivatives on a neighborhood of this point.

Esaim: Cocv, June 1997, Vol. 2, PP. 151-230 
Note that the fact that $\left\{\omega_{1}, \omega_{2}\right\}$ be a linearizing Pfaffian system implies that $\eta_{1}$ and $\eta_{2}$, or $\mathrm{d} h_{1}, \mathrm{~d} h_{2}$ are always linear combinations of $\omega_{1}, \omega_{2}$ and a finite number of their time derivatives.

Let us now translate this property into existence of an operator relating $\left(\omega_{1}, \omega_{2}\right)$ and $\left(\eta_{1}, \eta_{2}\right)$. For an open set $U$ in $\mathcal{M}_{\infty}^{2,4}$, let $\mathcal{A}(U)$ be the $\mathcal{C}^{\infty}(U)$ algebra:

$$
\mathcal{A}(U) \triangleq \mathcal{M}_{2 \times 2}\left(\mathcal{C}^{\infty}(U)\left[L_{F}\right]\right)
$$

of $2 \times 2$ matrices whose entries are differential operators, polynomial in the derivation along $F$, i.e. whose entries are of the form

$$
p_{0}+p_{1} \frac{\mathrm{d}}{\mathrm{dt}}+p_{2} \frac{\mathrm{d}}{\mathrm{dt}}^{2}+\cdots+p_{K} \frac{\mathrm{d}}{\mathrm{dt}}^{K},
$$

where the $p_{i}$ 's are smooth functions from $U$ to $\mathbb{R}$ (recall it means they depend only on $x$ and a finite number of time-derivatives of $u$ ). Elements of $\mathcal{A}(U)$ act in an obvious manner on pairs of functions, or on pairs of differential forms.

Proposition $2.9([1])$. Let $\left\{\omega_{1}, \omega_{2}\right\}$ be a linearizing Pfaffian system at a certain point. Then for two forms $\eta_{1}$ and $\eta_{2},\left\{\eta_{1}, \eta_{2}\right\}$ is a linearizing Pfaffian system if and only if on a neighborhood $U$ of this point, there exists $P \in \mathcal{A}(U)$ such that

$$
\left\{\begin{array}{l}
P \text { has an inverse in } \mathcal{A}(U), \\
\left(\begin{array}{l}
\eta_{1} \\
\eta_{2}
\end{array}\right)=P\left(\frac{\mathrm{d}}{\mathrm{dt}}\right)\left(\begin{array}{l}
\omega_{1} \\
\omega_{2}
\end{array}\right) .
\end{array}\right.
$$

This has some interest because it is possible, at least away from some singular points, to build a linearizing Pfaffian system for any accessible system. This is the construction of the "infinitesimal Brunovsky form" [2, 1]. Some sequences of modules (over smooth functions) of 1-forms and of vector fields, called $\mathcal{H}_{k}, \mathcal{D}_{k}$ and $\widehat{\mathcal{D}}_{k}$ are defined in [1]. Points where they have constant rank are called "Brunovský-regular", and at these points, a special linearizing Pfaffian system may be constructed. Let us recall here the minimum needed for our specific dimensions. Define the following modules of vector fields over smooth functions:

$$
\begin{aligned}
\widehat{\mathcal{D}}_{2}= & \operatorname{Span}\left\{X_{1}, X_{2}\right\} \\
\widehat{\mathcal{D}}_{3}= & \widehat{\mathcal{D}}_{2}+\left[F, \widehat{\mathcal{D}}_{2}\right] \\
= & \operatorname{Span}\left\{X_{1}, X_{2},\left[X_{0}, X_{1}\right]-u_{2}\left[X_{1}, X_{2}\right],\left[X_{0}, X_{2}\right]+u_{1}\left[X_{1}, X_{2}\right]\right\} \\
\widehat{\mathcal{D}}_{4}= & \widehat{\mathcal{D}}_{3}+\left[F, \widehat{\mathcal{D}}_{3}\right] \\
= & \operatorname{Span}\left\{X_{1}, X_{2},\left[X_{0}, X_{1}\right]-u_{2}\left[X_{1}, X_{2}\right],\left[X_{0}, X_{2}\right]+u_{1}\left[X_{1}, X_{2}\right],\right. \\
& {\left[X_{0},\left[X_{0}, X_{1}\right]\right]+u_{1}\left[X_{1},\left[X_{0}, X_{1}\right]\right]+u_{2}\left(\left[X_{1},\left[X_{0}, X_{1}\right]\right]-\left[X_{0},\left[X_{1}, X_{2}\right]\right]\right) } \\
& \quad-u_{1} u_{2}\left[X_{1},\left[X_{1}, X_{2}\right]\right]-u_{2}^{2}\left[X_{2},\left[X_{1}, X_{2}\right]\right]-\dot{u}_{2}\left[X_{1}, X_{2}\right], \\
& {\left[X_{0},\left[X_{0}, X_{2}\right]\right]+u_{1}\left(\left[X_{1},\left[X_{0}, X_{2}\right]\right]+\left[X_{1},\left[X_{0}, X_{2}\right]\right]\right)+u_{2}\left[X_{1},\left[X_{0}, X_{2}\right]\right] } \\
& \left.\quad+u_{1}^{2}\left[X_{1},\left[X_{1}, X_{2}\right]\right]+u_{1} u_{2}\left[X_{2},\left[X_{1}, X_{2}\right]\right]+\dot{u}_{1}\left[X_{1}, X_{2}\right]\right\} .
\end{aligned}
$$

Definition 2.10. A point $(x, u, \dot{u})$ where the vector fields $X_{1}$ and $X_{2}$ are not collinear is called Brunovsky regular if and only if the three distributions $\widehat{\mathcal{D}}_{2}, \widehat{\mathcal{D}}_{3}$ and $\widehat{\mathcal{D}}_{4}$ have constant rank in a neighborhood of this point. A point 
$(x, u, \dot{u}, \ddot{u}, \ldots) \in \mathcal{M}_{\infty}^{2,4}$ is called Brunovský regular if and only if the $(x, u, \dot{u})$ is Brunovský regular.

The fact that Brunovský regularity depends on the value of $x, u$ and $\dot{u}$ only comes from the fact that the vector fields in (2.9) depend on the eight variables $x, u, \dot{u}$ only (note also that they are linear combinations of the four coordinate vector fields corresponding to the $x$-coordinates only $\ldots$, they might be seen as vector fields on $\mathbb{R}^{4}$ parameterized by $u$ and $\dot{u}$ ).

We always assume that the rank of $\widehat{\mathcal{D}}_{2}$ is two, then, at a Brunovsky regular point, the ranks of $\widehat{\mathcal{D}}_{2}, \widehat{\mathcal{D}}_{3}, \widehat{\mathcal{D}}_{4}$ may only be 2,2 , 2, or $2,3,3$, or $2,3,4$, or $2,4,4$. In the two first cases, system (1.5) is not accessible (see [1]). In the two other cases, [1, theorem 2] allows to build a linearizing Pfaffian system $\left\{\omega_{1}, \omega_{2}\right\}$ which has the peculiarity that either $\left\{\omega_{1}, \dot{\omega}_{1}, \omega_{2}, \dot{\omega}_{2}\right\}$ or $\left\{\omega_{1}, \dot{\omega}_{1}, \ddot{\omega}_{1}, \omega_{2}\right\}$ is a basis of $\operatorname{Span}\{\mathrm{d} \mathbf{x}\}$ (see the meaning of $\operatorname{Span}\{\mathrm{d} \mathbf{x}\}$ at the end of section 2.2). Let us make this precise, only in the case where the ranks are 2, 4, 4 because we will not use this process in the case 2, 3, 4 .

Proposition 2.11 (Infinitesimal Brunovský Form [1]). Around a point where the ranks of $\widehat{\mathcal{D}}_{2}, \widehat{\mathcal{D}}_{3}$ and $\widehat{\mathcal{D}}_{4}$ are 2 , \& and 4 respectively, and if $\omega_{1}$ and $\omega_{2}$ are two linearly independent 1-forms in the annihilator of $\widehat{\mathcal{D}}_{2}$, i.e. of $\left\{X_{1}, X_{2}\right\}$ :

$$
\left\{\omega_{1}, \omega_{2}\right\}=\operatorname{Span}\{\mathbf{d x}\} \cap\left\{X_{1}, X_{2}\right\}^{\perp}
$$

then $\left\{\omega_{1}, \omega_{2}\right\}$ is a linearizing Pfaffian system, and more precisely, $\left\{\omega_{1}, \omega_{2}, \dot{\omega}_{1}\right.$, $\left.\dot{\omega}_{2}\right\}$ is a basis of $\operatorname{Span}\{\mathrm{dx}\},\left\{\omega_{1}, \omega_{2}, \dot{\omega}_{1}, \dot{\omega}_{2}, \ddot{\omega}_{1}, \ddot{\omega}_{2}\right\}$ is a basis of $\operatorname{Span}\{\mathrm{dx}$, $\mathrm{d} \mathbf{u}\}$, and more generally $\left\{\omega_{1}, \omega_{2}, \dot{\omega}_{1}, \dot{\omega}_{2}, \ldots, \omega_{1}^{(J)}, \omega_{2}^{(J)}\right\}$ is a basis of $\operatorname{Span}\left\{\mathrm{d} \mathbf{x}, \mathrm{d} \mathbf{u}, \mathrm{d} \dot{\mathbf{u}}, \ldots, \mathrm{d} \mathbf{u}^{(J-2)}\right\}$. The 1-forms $\omega_{1}$ and $\omega_{2}$ can be chosen involving $x$ only.

This is a particular case of [1, theorem 2]. The following proof may however help the reader's understanding.

Proof. The forms $\omega_{1}$ and $\omega_{2}$ satisfying (2.9) may always be chosen so that they involve $x$ only because $X_{1}$ and $X_{2}$ involve $x$ only. We use the following identity, which is true for any form $\omega$ and any vector field $X$ :

$$
\begin{aligned}
\langle\dot{\omega}, X\rangle & =\left\langle L_{F} \omega, X\right\rangle \\
& =L_{F}\langle\omega, X\rangle-\langle\omega,[F, X]\rangle \\
& =\frac{\mathrm{d}}{\mathrm{dt}}\langle\omega, X\rangle-\langle\omega,[F, X]\rangle .
\end{aligned}
$$

Now, on one hand the forms $\dot{\omega}_{1}$ and $\dot{\omega}_{2}$ are in $\operatorname{Span}\{\mathrm{dx}\}$, i.e. have no component on $\mathrm{d} u_{1}$ and $\mathrm{d}_{2}$ because (2.10) implies

$$
\left\langle\dot{\omega}_{k}, \frac{\partial}{\partial u_{i}}\right\rangle=\left\langle\omega_{k},\left[F, \frac{\partial}{\partial u_{i}}\right]\right\rangle=\left\langle\omega_{k}, X_{i}\right\rangle=0
$$

for $k=1,2$ and $i=1,2$. On the other hand, $\omega_{1}, \omega_{2}, \dot{\omega}_{1}, \dot{\omega}_{2}$ are linearly independent: if it was not that case at a point, there would exist some constants $\lambda_{1}, \lambda_{2}, \mu_{1}, \mu_{2}$, not all zero, such that $\lambda_{1} \dot{\omega}_{1}+\lambda_{2} \dot{\omega}_{2}+\mu_{1} \omega_{1}+\mu_{2} \omega_{2}$ would vanish at this point; since $\left\langle\omega_{1}, X_{i}\right\rangle=\left\langle\omega_{2}, X_{i}\right\rangle=0$, this would imply that, for $i=1,2,\left\langle\lambda_{1} \dot{\omega}_{1}+\lambda_{2} \dot{\omega}_{2}, X_{i}\right\rangle$ also vanish at this point; this in turn Esaim: Cocv, June 1997, Vol. 2, Pr. 151-230 
would imply, from identity (2.10), that

$$
\left\langle\lambda_{1} \omega_{1}+\lambda_{2} \omega_{2},\left[X_{0}+u_{1} X_{1}+u_{2} X_{2}, X_{i}\right]\right\rangle
$$

vanishes at this point, i.e. that $\lambda_{1} \omega_{1}+\lambda_{2} \omega_{2}$ is in the annihilator of $\widehat{\mathcal{D}}_{3}$, and hence that $\lambda_{1}=\lambda_{2}=0$ because the rank of $\widehat{\mathcal{D}}_{3}$ is 4 and $\omega_{1}$ and $\omega_{2}$ are independent; this is impossible because then $\mu_{1} \omega_{1}+\mu_{2} \omega_{2}$ would vanish at the considered point while $\omega_{1}$ and $\omega_{2}$ are independent. It is easy to prove the last property for all $J \geq 2$ : since $\omega_{1}, \omega_{2}, \dot{\omega}_{1}, \dot{\omega}_{2}$ are in $\operatorname{Span}\{\mathrm{dx}\}$ and $\mathrm{d} u_{k}^{(\ell+1)}$ may only appear by taking the time-derivative of $\mathrm{d} u_{k}^{(\ell)}$, it is clear that $\omega_{k}^{(j)}$ is in $\operatorname{Span}\left\{\mathrm{d} x, \mathrm{~d} u, \mathrm{~d} \dot{u}, \ldots, \mathrm{d} u^{(j-2)}\right\}$, and the linear independence of all these is proved by using recursively identity (2.10).

The term "infinitesimal Brunovsky form" refers to the fact that, with the above choices of the 1 -forms $\omega_{1}$ and $\omega_{2}$, system (1.5) implies:

$$
\begin{aligned}
\frac{d}{d t} \omega_{1} & =\dot{\omega}_{1} \\
\frac{d}{d t} \dot{\omega}_{1} & =\sum_{1}^{4} \alpha_{1, i} \mathrm{~d} x_{i}+\beta_{1,1} \mathrm{~d} u_{1}+\beta_{1,2} \mathrm{~d} u_{2} \\
\frac{d}{d t} \omega_{2} & =\dot{\omega}_{2} \\
\frac{d}{d t} \dot{\omega}_{2} & =\sum_{1}^{4} \alpha_{2, i} \mathrm{~d} x_{i}+\beta_{2,1} \mathrm{~d} u_{1}+\beta_{2,2} \mathrm{~d} u_{2}
\end{aligned}
$$

where the functions $\beta_{i, j}$ are such that the $2 \times 2$ matrix $\left[\beta_{i, j}\right]$ is invertible on a neighborhood of $(\bar{x}, \bar{u})$. If the forms $\omega_{1}$ and $\omega_{2}$ were integrable, one might define $z$ function of $x$ and $v$ function of $x, u$ (static feedback transformation) by $\mathrm{d} z_{1}=\omega_{1}, \mathrm{~d} z_{2}=\dot{\omega}_{1}, \mathrm{~d} v_{1}=\ddot{\omega}_{1}, \mathrm{~d} z_{3}=\omega_{2}, \mathrm{~d} z_{4}=\dot{\omega}_{2}, \mathrm{~d} v_{2}=\ddot{\omega}_{2}$, such that (1.5) reads like the Brunovský canonical form (2.3.b) - we would have obtained the form (2.3.b) if we would have considered the case where the ranks of $\widehat{\mathcal{D}}_{2}, \widehat{\mathcal{D}}_{3}, \widehat{\mathcal{D}}_{4}$ are $2,3,4$-. It is called "infinitesimal" because it is only at the level of differential forms instead of functions (coordinates) and can give functions if the differential forms are integrable, which is false in general.

Now that we have built a special linearizing Pfaffian system, we may state the following consequence of propositions $2.7,2.9$ and 2.11 . It is specialized to $x$-dynamic linearization or $(x, u)$-dynamic linearization, and the fact that the linearizing outputs depend on $x$ only or on $x$ and $u$ only is translated into a condition on the degree of the entries of the matrix $P$ comes from the special properties on $\omega_{1}$ and $\omega_{2}$ given in proposition 2.11. Again, this is only stated in the case where the ranks of $\widehat{\mathcal{D}}_{2}, \widehat{\mathcal{D}}_{3}$ and $\widehat{\mathcal{D}}_{4}$ are 2,4 , 4 because we will not use this process in the case $2,3,4$.

Proposition 2.12 ([19]). Let $(\bar{x}, \bar{u})$ be a point where the ranks of $\widehat{\mathcal{D}}_{2}, \widehat{\mathcal{D}}_{3}$ and $\widehat{\mathcal{D}}_{4}$ are $2,4,4$, and $\omega_{1}$ and $\omega_{2}$ be defined in a neighborhood of $(\bar{x}, \bar{u})$ as in proposition 2.11 (see equation (2.9)). System (1.5) is $x$-dynamic linearizable (resp. $(x, u)$-dynamic linearizable) at point $\left(\bar{x}, \bar{u}, \ldots, \bar{u}^{(J)}\right)$ if and only if there exists a neighborhood $U$ of this point, and a $2 \times 2$ polynomial matrix $P \in \mathcal{A}(U)$ whose entries are polynomials of degree at most 1 (resp. at most 2), such that $P$ has an inverse in $\mathcal{A}(U)$ and the Pfaffian system $\left\{\eta_{1}, \eta_{2}\right\}$ defined by

$$
\left(\begin{array}{l}
\eta_{1} \\
\eta_{2}
\end{array}\right)=P\left(\frac{\mathrm{d}}{\mathrm{dt}}\right)\left(\begin{array}{l}
\omega_{1} \\
\omega_{2}
\end{array}\right) .
$$


is completely integrable, i.e. $\eta_{1}$ and $\eta_{2}$ satisfy $\mathrm{d} \eta_{1} \wedge \eta_{1} \wedge \eta_{2}=\mathrm{d} \eta_{2} \wedge \eta_{1} \wedge \eta_{2}=0$ in a neighborhood of this point.

We shall use this property, especially for $(x, u)$-dynamic linearizability in section 4. Of course, this would be useless without a reasonable description of the invertible matrices in $\mathcal{A}(U)$ of degree at most 2. In fact, away from some singularities, invertible matrices may be described as products of "elementary matrices", like unimodular matrices in the case of polynomials with constant coefficients:

Proposition 2.13. Let $P$ be a matrix in $\mathcal{A}(U)$, which has an inverse $Q$ in $\mathcal{A}(U)$.

(i) If the degree of $P$ is 1 on an open dense subset of $U$ (i.e. $P$ has degree at most 1 everywhere, and possibly zero on a closed set of empty interior), then there is an open dense subset $U_{0}$ of $U$ such, for that all $\mathcal{X} \in U_{0}$, there is a neighborhood $V_{\mathcal{X}}$, a scalar smooth function a, and two invertible matrices $J_{1}$ and $J_{2}$ of degree 0 (i.e. whose entries are smooth functions), all defined on $V_{\mathcal{X}}$, such that, on $V_{\mathcal{X}}$,

$$
P\left(\frac{\mathrm{d}}{\mathrm{dt}}\right)=J_{1}\left(\begin{array}{cc}
1 & -a \frac{\mathrm{d}}{\mathrm{dt}} \\
0 & 1
\end{array}\right) J_{2} .
$$

(ii) If the degree of $P$ is 2 on an open dense subset of $U$ (i.e. $P$ has degree at most 2 everywhere, and possibly 1 or 0 on a closed set of empty interior), then there is an open dense subset $U_{0}$ of $U$ such, for that all $\mathcal{X} \in U_{0}$, there is a neighborhood $V_{\mathcal{X}}$, scalar smooth functions $\alpha, \lambda$, a and $b$, and an invertible matrix $J_{1}$ of degree $O$ (i.e. whose entries are smooth functions), all defined on $V_{\mathcal{X}}$, such that, on $V_{\mathcal{X}}$, either

$$
P\left(\frac{\mathrm{d}}{\mathrm{dt}}\right)=J_{1}\left(\begin{array}{cc}
1 & -a \frac{\mathrm{d}}{\mathrm{dt}} \\
0 & 1
\end{array}\right)\left(\begin{array}{cc}
1 & 0 \\
-b \frac{\mathrm{d}}{\mathrm{dt}} & 1
\end{array}\right) J_{2}
$$

or

$$
P\left(\frac{\mathrm{d}}{\mathrm{dt}}\right)=J_{1}\left(\begin{array}{cc}
1 & 0 \\
-a \frac{\mathrm{d}}{\mathrm{dt}}-b \frac{\mathrm{d}}{\mathrm{dt}}^{2} & 1
\end{array}\right) J_{2}
$$

with

$$
\text { either } J_{2}=\left(\begin{array}{ll}
1 & 0 \\
\alpha & 1
\end{array}\right)\left(\begin{array}{ll}
\lambda & 1 \\
1 & 0
\end{array}\right) \quad \text { or } J_{2}=\left(\begin{array}{ll}
1 & 0 \\
\alpha & 1
\end{array}\right) \text {. }
$$

Proof. Although the ring of polynomials $\mathcal{C}^{\infty}(U)\left[\frac{\mathrm{d}}{\mathrm{dt}}\right]$ is not commutative, there is a left and right Euclidean division by polynomials whose leading coefficient does not vanish (this is because the leading coefficient of the product of two polynomials is computed as if the coefficients were constant). We also use the fact that the matrix formed with the coefficients of the terms of higher degree on each column cannot be invertible for an invertible matrix, except if it is a degree zero matrix.

(i) For the case of degree 1, at points where not all leading coefficients vanish, there is an invertible matrix $K_{2}$ of degree zero (may be take either triangular or a permutation matrix) such that $P\left(\frac{\mathrm{d}}{\mathrm{d} t}\right) K_{2}$ has its first column of degree zero. Then at points where not both terms of this column vanish, a Euclidean division yields a smooth function a such that Esaim: Cocv, June 1997, Vol. 2, Pr. 151-230 
$J_{1}=P\left(\frac{\mathrm{d}}{\mathrm{d} t}\right) K_{2}\left(\begin{array}{cc}1 & -a \frac{\mathrm{d}}{\mathrm{dt}} \\ 0 & 1\end{array}\right)$ has degree zero. Take $J_{2}=K_{2}^{-1}$. The open set $U_{0}$ is the set where the functions we had to divide by do not vanish.

(ii) For the case of degree 2, let us distinguish different cases. In all cases, we have to divide by at most three polynomials, the points where they vanish without being zero on a neighborhood - if they are zero on an open set, then the corresponding polynomial has locally a smaller degree- is closed with empty interior, the open set $U_{0}$ is its complement.

- If both polynomials in the second column of $P\left(\frac{\mathrm{d}}{\mathrm{dt}}\right)$ have degree zero, then, at any point, one of them at least does not vanish, and dividing by it the corresponding polynomial (degree 2 ) in the first column yields a degree two polynomial $-\alpha+a \frac{\mathrm{d}}{\mathrm{dt}}+b \frac{\mathrm{d}}{\mathrm{dt}}^{2}$ such that

$$
j_{1}=P\left(\frac{\mathrm{d}}{\mathrm{dt}}\right)\left(\begin{array}{cc}
1 & 0 \\
-\alpha+a \frac{\mathrm{d}}{\mathrm{dt}}+b \frac{\mathrm{d}}{\mathrm{dt}}^{2} & 1
\end{array}\right)
$$

has degree zero. This yields (2.14) with the second expression for $J_{2}$ in $(2.15)$.

- If both polynomials in the second column of $P\left(\frac{\mathrm{d}}{\mathrm{dt}}\right)$ have degree at most 1 but they are not both of degree zero, then, at any point where the leading coefficient of this one does not vanish, Euclidean division by this polynomial of the corresponding polynomial (degree 2) in the first column yields a degree one polynomial $-\alpha+b \frac{\mathrm{d}}{\mathrm{dt}}$ such that

$$
P\left(\frac{\mathrm{d}}{\mathrm{dt}}\right)\left(\begin{array}{cc}
1 & 0 \\
-\alpha+b \frac{\mathrm{d}}{\mathrm{dt}} & 1
\end{array}\right)
$$

has a first column of degree zero, and then dividing by a non-vanishing element of this first column yields $a$ such that

$$
J_{1}=P\left(\frac{\mathrm{d}}{\mathrm{dt}}\right)\left(\begin{array}{cc}
1 & 0 \\
-\alpha+b \frac{\mathrm{d}}{\mathrm{dt}} & 1
\end{array}\right)\left(\begin{array}{cc}
1 & a \frac{\mathrm{d}}{\mathrm{dt}} \\
0 & 1
\end{array}\right)
$$

has degree zero. This yields (2.13) with the second expression for $J_{2}$ in $(2.15)$.

- If at least one of the polynomials in the second column of $P\left(\frac{d}{d t}\right)$ has degree 2, then, at points where its leading coefficient does not vanish, dividing the corresponding polynomial in the first column by this coefficient yields a function $\lambda$ such that

$$
P\left(\frac{\mathrm{d}}{\mathrm{dt}}\right)\left(\begin{array}{cc}
0 & 1 \\
1 & -\lambda
\end{array}\right)
$$

has both entries in its second column of degree at most 1 ( $\lambda$ is identically zero if the first column of $P\left(\frac{\mathrm{d}}{\mathrm{dt}}\right)$ had degree 1 or 0$)$. Apply one of the two first cases to $P\left(\frac{\mathrm{d}}{\mathrm{d} t}\right)\left(\begin{array}{cc}0 & 1 \\ 1 & -\lambda\end{array}\right)$ instead of $P\left(\frac{\mathrm{d}}{\mathrm{d} t}\right)$. This yields either (2.14) or (2.13), with the first expression for $J_{2}$ in (2.15). 


\subsection{Two WAYS OF WRITING THE EQUATIONS FOR THE LINEARIZING OUTPUTS}

The most natural method for deciding if there exists some linearizing outputs depending on $x$ and $u$ is to write down the equations that a pair of functions has to satisfy in order to be a pair of linearizing outputs, and then to find conditions (on the system (1.5)) for these equations to have solutions. Let us describe these equations, but only for the case when the linearizing outputs are restricted to depend upon $x$ only:

Proposition 2.14. Suppose that $X_{1}$ and $X_{2}$ in (1.5) or (2.5) are linearly independent. Let $h_{1}(x)$ and $h_{2}(x)$ be smooth functions; then $\left(h_{1}, h_{2}\right)$ is a pair of linearizing outputs at a certain point if and only if

$$
\begin{gathered}
\operatorname{rank}\left(\begin{array}{ll}
\frac{\partial \dot{h}_{1}}{\partial u_{1}} & \frac{\partial \dot{h}_{1}}{\partial u_{2}} \\
\frac{\partial \dot{h}_{2}}{\partial u_{1}} & \frac{\partial \dot{h}_{2}}{\partial u_{2}}
\end{array}\right) \leq 1 \\
\operatorname{rank}\left(\begin{array}{cccc}
\frac{\partial \dot{h}_{1}}{\partial u_{1}} & \frac{\partial \dot{h}_{1}}{\partial u_{2}} & 0 & 0 \\
\frac{\partial \dot{h}_{2}}{\partial u_{1}} & \frac{\partial \dot{h}_{2}}{\partial u_{2}} & 0 & 0 \\
\frac{\partial \ddot{h}_{1}}{\partial u_{1}} & \frac{\partial \ddot{h}_{1}}{\partial u_{2}} & \frac{\partial \ddot{h}_{1}}{\partial \dot{u}_{1}} & \frac{\partial \ddot{h}_{1}}{\partial \dot{u}_{2}} \\
\frac{\partial \ddot{h}_{2}}{\partial u_{1}} & \frac{\partial \ddot{h}_{2}}{\partial u_{2}} & \frac{\partial \dot{h}_{2}}{\partial \dot{u}_{1}} & \frac{\partial \ddot{h}_{2}}{\partial \dot{u}_{2}}
\end{array}\right) \leq 2
\end{gathered}
$$

on a neighborhood of this point, and the forms $\mathrm{d} h_{1}, \mathrm{~d} h_{2}, \mathrm{~d} \dot{h}_{1}, \mathrm{~d} \dot{h}_{2}, \mathrm{~d} \ddot{h}_{1}, \mathrm{~d} \ddot{h}_{2}$ are independent at this point.

Proof. Let us prove necessity. If $\left(h_{1}, h_{2}\right)$ is a pair of linearizing outputs, the six mentioned forms have to be independent by definition. If the rank in (2.16) was 2 , it is clear that the only linear combinations of the $\mathrm{d} h_{k}^{(j)}$, $\mathrm{s}$ which would also be linear combinations of $\mathrm{d} x_{1}, \mathrm{~d} x_{2}, \mathrm{~d} x_{3}, \mathrm{~d} x_{4}$, would have all their coefficients zero except the coefficients of $\mathrm{d} h_{1}$ and $\mathrm{d} h_{2}$, which would contradict the fact that $\mathrm{d} x_{1}, \mathrm{~d} x_{2}, \mathrm{~d} x_{3}$ and $\mathrm{d} x_{4}$ are linear combinations of the $\mathrm{d} h h_{k}^{(j)}$ s. This proves that (2.16) is necessary. If the rank in (2.17) was 3 (cannot be 4 from (2.16)), the only linear combinations of the $\mathrm{d} h_{k}^{(j)}$ s which would also be also linear combinations of $\mathrm{d} x_{1}, \mathrm{~d} x_{2}, \mathrm{~d} x_{3}, \mathrm{~d} x_{4}$, would be linear combinations of $\mathrm{d} h_{1}, \mathrm{~d} h_{2}$ and $\lambda_{1} \mathrm{~d} \dot{h}_{1}+\lambda_{2} \mathrm{~d} \dot{h}_{2}$ with the line $\left(\lambda_{1}, \lambda_{2}\right)$ in the right kernel of the matrix in (2.17), impossible from the fact that contradict the fact that $\mathrm{d} x_{1}, \mathrm{~d} x_{2}, \mathrm{~d} x_{3}$ and $\mathrm{d} x_{4}$ are independent linear combinations of the $\mathrm{d} h_{k}^{(j)}$ s. This proves that (2.17) is necessary. which are also linear combinations of $\mathrm{d} x_{1}, \mathrm{~d} x_{2}$, Sufficiency follows from solving for $\mathrm{d} x_{1}, \mathrm{~d} x_{2}, \mathrm{~d} x_{3}$ and $\mathrm{d} x_{4}$ as linear combinations of $\mathrm{d} h_{1}, \mathrm{~d} h_{2}, \mathrm{~d} \dot{h}_{1}, \mathrm{~d} \dot{h}_{2}, \mathrm{~d} \ddot{h}_{1}$ and $\mathrm{d} \ddot{h}_{2}$.

Conditions (2.16)-(2.17) are better related to the vector fields defining system (1.5) using:

and

$$
\frac{\partial \ddot{h}_{i}}{\partial \dot{u}_{k}}=\frac{\partial \dot{h}_{i}}{\partial u_{k}}=L_{X_{k}} h_{i}
$$

$$
\begin{aligned}
\frac{\partial \ddot{h}_{i}}{\partial u_{k}}= & L_{X_{0}} L_{X_{k}} h_{i}+L_{X_{k}} L_{X_{0}} h_{i}+2 u_{k} L_{X_{k}}^{2} h_{i} \\
& +u_{k^{\prime}}\left(L_{X_{k^{\prime}}} L_{X_{k}} h_{i}+L_{X_{k}} L_{X_{k^{\prime}}} h_{i}\right)
\end{aligned}
$$


where $k^{\prime}=2$ if $k=1$ and $k^{\prime}=1$ if $k=2$.

The two equations (2.16)-(2.17) give a system of PDEs in $h_{1}$ and $h_{2}$ (some determinants being zero), and the independence condition an inequality (a nonzero determinant). These have solutions of and only if the system is $x$-dynamic linearizable.

Some similar conditions on functions of $x$ and $u$ may be written, and existence of solution would be equivalent for $(x, u)$-dynamic linearizability.

A different possibility is to use the material introduced in section 2.5: under non-singularity conditions (being at a "Brunovský regular" point), there exists two differential forms such that $\left\{\omega_{1}, \omega_{2}, \dot{\omega}_{1}, \dot{\omega}_{2}\right\}$ (or $\left\{\omega_{1}, \dot{\omega}_{1}, \ddot{\omega}_{1}, \omega_{2}\right\}$ but let us consider the first case only) is a basis of $\operatorname{Span}\{\mathrm{dx}\}$, these forms may be constructed explicitly, and, from proposition 2.12 , the system is $x$-linearizable or $(x, u)$-dynamic linearizable if and only if there exists an invertible polynomial matrix such that

$$
P\left(\frac{\mathrm{d}}{\mathrm{dt}}\right)\left(\begin{array}{c}
\omega_{1} \\
\omega_{2}
\end{array}\right)
$$

is made of two exact one-forms, with some bounds on the degree of the entries of $P$. We then translate the fact that these forms are exact into a system of PDEs in the coefficients of the matrix, using the decomposition from proposition 2.13 . The system is $x$-dynamic or $(x, u)$-dynamic linearizable if and only if these PDEs have solutions.

These two methods - writing directly the PDEs a pair of functions has to satisfy to be a pair of linearizing outputs or writing the PDEs the coefficients of the elementary matrices in the decomposition of $P$ have to satisfy for the Pfaffian system $P\left(\frac{\mathrm{d}}{\mathrm{dt}}\right)\left(\omega_{1}, \omega_{2}\right)^{T}$ to be integrable- are obviously equivalent, although they lead to different equations.

One drawback of the second method is that it only works at "Brunovsky'regular" points, while Brunovský-regularity is not necessary for dynamic feedback linearization, see the example in section 5. Although Brunovskýregular points form an open dense set, one cannot neglect this weakness. Note however that in the example of section 5, we conclude even at points which are not Brunovský-regular, by density. In general, this second method seems to yield equations that may be considered more geometrically, and it proves to be very useful in our proofs.

For the simplest cases (cases 1 to 5 in theorem 3.1 ), we have used the first (direct) method, or even no particular method from these when we simply exhibit some pairs of linearizing outputs. Case 6 in theorem 3.1 is not elementary; it contains a necessary condition that we prove using the first (direct) method; the proof is natural; it would also be in a sense simpler using the infinitesimal Brunovsky form, but this case would then be split into two because depending whether (4.5) holds or not, the infinitesimal Brunovsky form is different, and points on the boundary are not Brunovskýregular while the present proof has no problem at these points. We give as an alternative a proof based on the infinitesimal Brunovský form, outside singularities (section 7.1 ). To test for $(x, u)$-linearizability, we were not able to use the direct method, and we had to use the second one based on infinitesimal Brunovsky form. It turns out that the first one yield rather huge PDEs in the linearizing outputs, and we found no obvious way to 
handle them naturally as in the case of $x$-dynamic linearization, while the second one gives some PDEs that, though very heavy computations are needed, may be handled by elementary methods.

\section{3. $x$-DYNAMIC LINEARIZABILITY}

We define the following distributions

$$
\begin{aligned}
\Delta_{2} & =\operatorname{Span}\left\{X_{1}, X_{2}\right\} \\
\mathcal{M}_{0} & =\Delta_{2}+\left[\Delta_{2}, \Delta_{2}\right]=\operatorname{Span}\left\{X_{1}, X_{2},\left[X_{1}, X_{2}\right]\right\} \\
\mathcal{M}_{1} & =\mathcal{M}_{0}+\left[\mathcal{M}_{0}, \mathcal{M}_{0}\right] \\
& =\operatorname{Span}\left\{X_{1}, X_{2},\left[X_{1}, X_{2}\right],\left[X_{1},\left[X_{1}, X_{2}\right]\right],\left[X_{2},\left[X_{1}, X_{2}\right]\right]\right\} \\
\Delta_{3} & =\operatorname{Span}\left\{X_{1}, X_{2},\left[X_{1}, X_{2}\right],\left[X_{0}, X_{1}\right],\left[X_{0}, X_{2}\right]\right\} .
\end{aligned}
$$

We will only study the situation in the neighborhood of points where the rank these distributions are constant, and the vector fields $X_{1}$ and $X_{2}$ are linearly independent and we define the integers $m_{0}, m_{1}, \delta_{3}$ by:

$$
\begin{aligned}
\operatorname{rank} \Delta_{2}=2 & \delta_{3} \triangleq \operatorname{rank} \Delta_{3} \\
& m_{0} \triangleq \operatorname{rank} \mathcal{M}_{0} \\
m_{1} & \triangleq \operatorname{rank} \mathcal{M}_{1} .
\end{aligned}
$$

These ranks and the distributions in (3.1) a re obviously feedback invariant from their definition and (2.2).

At a point where these ranks are constant, the only possible values for $\left(m_{0}, m_{1}, \delta_{3}\right)$ are $(2,2,2),(2,2,3),(2,2,4),(3,3,3),(3,3,4),(3,4,3)$ and $(3,4,4)$. Actually, we will not distinguish between cases $(3,4,3)$ and $(3,4,4)$, so that when $\left(m_{0}, m_{1}\right)=(3,4)$, the rank of $\Delta_{3}$ need not be constant.

The following theorem allows one, in each of the cases depending on the different possible values of the above ranks, to decide whether system (1.5) is $x$-dynamic linearizable or not. When it is not only $x$-dynamic linearizable, but static feedback linearizable, this is mentioned. In addition, for each case, we give a normal form for system (1.5) up to a nonsingular static feedback transformation (see (2.1)) and a change of coordinates. The proof is given in section 7.1. A small package written in Maple that makes the needed computations, as well as these corresponding to theorem 4.2 if needed, will soon be available from the author; it is described in [16].

THEOREM 3.1. Let $\bar{x}$ be such that the distributions spanned by the modules $\Delta_{2}, \mathcal{M}_{0}, \mathcal{M}_{1}$ and $\Delta_{3}$ have constant rank in a neighborhood of $\bar{x}$, with $\Delta_{2}$ of rank 2, as in (3.2). Actually, if $\left(m_{0}, m_{1}\right)=(3,4)$, we do not require that the rank of $\Delta_{3}$ be constant.

1. If $m_{0}=m_{1}=2$ and $\delta_{3}=2$, system (1.5) is locally non accessible and therefore non linearizable by endogenous feedback. Locally around $\bar{x}$, after a preliminary nonsingular feedback transformation and in appropriate coordinates, it has the following form, where $a_{1}$ and $a_{2}$ are smooth functions:

$$
\begin{aligned}
& \dot{z}_{1}=a_{1}\left(z_{1}, z_{2}\right) \\
& \dot{z}_{2}=a_{2}\left(z_{1}, z_{2}\right) \\
& \dot{z}_{3}=v_{1} \\
& \dot{z}_{4}=v_{2} .
\end{aligned}
$$


2. If $m_{0}=m_{1}=2$ and $\delta_{3}=3$, there are three sub-cases:

(a) If $\Delta_{3}$ is not involutive (i.e. if there are points $x$ arbitrarily close to $\bar{x}$ such that $\left[\Delta_{3}, \Delta_{3}\right](x) \not \subset \Delta_{3}(x)$, even if $\left.\left[\Delta_{3}, \Delta_{3}\right](\bar{x}) \subset \Delta_{3}(\bar{x})\right)$, system (1.5) is not linearizable by endogenous dynamic feedback. It has locally, around $\bar{x}$, after a preliminary nonsingular feedback transformation and in appropriate coordinates, the following form:

$$
\begin{aligned}
& \dot{z}_{1}=a\left(z_{1}, z_{2}, z_{3}\right) \\
& \dot{z}_{2}=z_{3} \\
& \dot{z}_{3}=v_{1} \\
& \dot{z}_{4}=v_{2}
\end{aligned}
$$

where $a$ is a smooth function such that

$$
\frac{\partial^{2} a}{\partial z_{3}^{2}} \text { is not identically zero on any neighborhood of } \bar{x} \text {. }
$$

(b) If $\Delta_{3}$ involutive and the rank of $\Delta_{3}+\left[X_{0}, \Delta_{3}\right]$ is 3 in a neighborhood of $\bar{x}$, system (1.5) is locally non accessible and therefore non linearizable by endogenous feedback. Locally around $\bar{x}$, after a preliminary nonsingular feedback transformation and in appropriate coordinates, it has the following form, with a a smooth:

$$
\begin{aligned}
& \dot{z}_{1}=a\left(z_{1}\right) \\
& \dot{z}_{2}=z_{3} \\
& \dot{z}_{3}=v_{1} \\
& \dot{z}_{4}=v_{2} .
\end{aligned}
$$

(c) If $\Delta_{3}$ involutive and the rank of $\Delta_{3}+\left[X_{0}, \Delta_{3}\right]$ is \& at point $\bar{x}$ (and therefore in a neighborhood), system (1.5) is locally static feedback linearizable. It has, after a preliminary nonsingular feedback transformation and in appropriate coordinates, the form (2.3.a).

3. If $m_{0}=m_{1}=2$ and $\delta_{3}=4$, system (1.5) is locally static feedback linearizable. It has, after a preliminary nonsingular feedback transformation and in appropriate coordinates, the form (2.3.b).

4. If $m_{0}=m_{1}=3$ and $\delta_{3}=3$, system (1.5) is locally non accessible and therefore non linearizable by endogenous feedback. Locally around $\bar{x}$, after a preliminary nonsingular feedback transformation and in appropriate coordinates, it has the following form, where $a_{1}$ and $a_{3}$ are smooth functions:

$$
\begin{aligned}
& \dot{z}_{1}=a_{1}\left(z_{1}\right) \\
& \dot{z}_{2}=v_{1} \\
& \dot{z}_{3}=a_{3}\left(z_{1}, z_{2}, z_{3}, z_{4}\right)+z_{4} v_{1} \\
& \dot{z}_{4}=v_{2} .
\end{aligned}
$$

5. If $m_{0}=m_{1}=3$ and $\delta_{3}=4$, system (1.5) is locally $x$-dynamic linearizable at a point $\left(\bar{x}, \bar{u}_{1}, \bar{u}_{2}, \ldots\right)$ if and only if

$$
\begin{array}{r}
\operatorname{rank}_{\mathbb{R}}\left\{X_{1}(\bar{x}), X_{2}(\bar{x}),\left[X_{0}, X_{1}\right](\bar{x})-\bar{u}_{2}\left[X_{1}, X_{2}\right](\bar{x}),\right. \\
\left.\left[X_{0}, X_{2}\right](\bar{x})+\bar{u}_{1}\left[X_{1}, X_{2}\right](\bar{x})\right\}=4 .
\end{array}
$$

This condition is satisfied on an open dense set of any open set where $m_{0}=m_{1}=3$ and $\delta_{3}=4$. 
After a preliminary nonsingular feedback transformation and in appropriate coordinates, the system has the following form:

$$
\begin{aligned}
& \dot{z}_{1}=z_{2} \\
& \dot{z}_{2}=v_{1} \\
& \dot{z}_{3}=a_{3}\left(z_{1}, z_{2}, z_{3}, z_{4}\right)+z_{4} v_{1} \\
& \dot{z}_{4}=v_{2}
\end{aligned}
$$

with $a$ is a smooth function. A possible choice of linearizing outputs is given, in these coordinates, by $h_{1}=z_{1}, h_{2}=z_{3}$. Condition (3.8) reads:

$$
v_{1}+\frac{\partial a_{3}}{\partial z_{4}} \neq 0
$$

6. If $m_{0}=3$ and $m_{1}=4$, there exists a unique (up to a nonzero multiplicative function) linear combination of $X_{1}$ and $X_{2}: \widetilde{X}=\lambda_{1} X_{1}+\lambda_{2} X_{2}$ such that

$$
\left[\tilde{X},\left[X_{1}, X_{2}\right]\right] \in \operatorname{Span}\left\{X_{1}, X_{2},\left[X_{1}, X_{2}\right]\right\}
$$

(this is the characteristic vector field, or characteristic direction of the distribution spanned by the independent vector fields $X_{1}, X_{2}$ and $\left.\left[X_{1}, X_{2}\right]\right)$.

System (1.5) is $x$-dynamic linearizable at $(\bar{x}, \bar{u})$ if and only if

$$
\left[\tilde{X}, X_{0}\right] \in \operatorname{Span}\left\{X_{1}, X_{2},\left[X_{1}, X_{2}\right]\right\}
$$

on a neighborhood of $\bar{x}$ and

$$
\begin{aligned}
& \operatorname{rank}_{\mathbb{R}}\left\{X_{1}(\bar{x}), X_{2}(\bar{x}),\left[X_{0}, \tilde{X}\right](\bar{x})+\bar{u}_{1}\left[X_{1}, \tilde{X}\right](\bar{x})\right. \\
& \left.\quad+\bar{u}_{2}\left[X_{2}, \tilde{X}\right](\bar{x})\right\}=3 \\
& \operatorname{rank}_{\mathbb{R}}\left\{X_{1}(\bar{x}), X_{2}(\bar{x}),\left[X_{1}, X_{2}\right](\bar{x}),\left[X_{0}, X_{1}\right](\bar{x}),\right. \\
& \quad\left[X_{0}, X_{2}\right](\bar{x}),\left[X_{0},\left[X_{1}, X_{2}\right]\right](\bar{x})+\bar{u}_{1}\left[X_{1},\left[X_{1}, X_{2}\right]\right](\bar{x}) \\
& \left.\quad+\bar{u}_{2}\left[X_{2},\left[X_{1}, X_{2}\right]\right](\bar{x})\right\}=4 .
\end{aligned}
$$

Given any open set in $\mathbb{R}^{4} \times \mathbb{R}^{2}$ such that for all $(\bar{x}, \bar{u})$ in this open set, $\left(m_{0}, m_{1}\right)=(3,4)$ and (3.12) is satisfied at $\bar{x}$, the set of $(\bar{x}, \bar{u})$ 's in this open set where (3.13) and (3.15) are satisfied is open and dense.

These conditions may also be formulated using differential forms instead of vector fields. Since $m_{0}=3$, one may take a-unique up to a nonzero multiplicative function-differential form in the four variables $x$ only annihilating $X_{1}, X_{2}$ and $\left[X_{1}, X_{2}\right]$ :

$$
\omega_{1} \in\left\{X_{1}, X_{2},\left[X_{1}, X_{2}\right]\right\}^{\perp}
$$

then $\mathrm{d} \omega_{1} \wedge \omega_{1}$ is a form of degree 3 that does not vanish because $m_{1}=4$. System (1.5) is $x$-dynamic linearizable at $(\bar{x}, \bar{u})$ if and only if

$$
\mathrm{d} \omega_{1} \wedge \omega_{1} \wedge \dot{\omega}_{1}=0
$$

on a neighborhood of $\bar{x}$ and

$$
\begin{aligned}
\operatorname{rank}_{\mathbb{R}}\left\{\omega_{1}(\bar{x}), \eta_{1}(\bar{x}), \eta_{2}(\bar{x}), \dot{\eta}_{1}(\bar{x}, \bar{u}), \dot{\eta}_{2}(\bar{x}, \bar{u})\right\} & =5, \\
\operatorname{rank}_{\mathbb{R}}\left\{\omega_{1}(\bar{x}), \dot{\omega}_{1}(\bar{x}, \bar{u})\right\} & =2,
\end{aligned}
$$


where $\eta_{1}$ and $\eta_{2}$ are forms of degree 1 such that, for a certain 1-form $\Gamma$,

$$
\mathrm{d} \omega_{1}=\omega_{1} \wedge \Gamma+\eta_{1} \wedge \eta_{2}
$$

or in other words $\mathrm{d} \omega_{1} \wedge \omega_{1}=\eta_{1} \wedge \eta_{2} \wedge \omega_{1} \quad\left\{\omega_{1}, \eta_{1}, \eta_{2}\right\}$ is the characteristic system of $\omega_{1}$, it is the annihilator of the vector field $\tilde{X}$ defined in (3.11)), and the "dot" is the time-derivative along the system, i.e. the Lie derivative along the vector field $F$ (2.5). The two conditions (3.17) and (3.18) are satisfied on an open dense set of any open set where $m_{0}=3$ and $m_{1}=4$.

When these conditions are met, all pairs of linearizing outputs may be obtained as follows: take for $h_{1}$ a first integral of the vector field $\widetilde{X}$ (i.e. $L_{\tilde{X}} h_{1}=0$ ) such that $\mathrm{d} h, \omega_{1}$ and $\dot{\omega}_{1}$ are linearly independent. Then the Pfaffian system $\left\{\mathrm{d} h_{1}, \omega_{1}\right\}$ is integrable. Take for $h_{2}$ a second first integral of this Pfaffian system.

Around a point where $\left(m_{0}, m_{1}\right)=(3,4)$, after a preliminary static feedback transformation (2.1) and in appropriate coordinates, system (1.5) has the form:

$$
\begin{aligned}
& \dot{z}_{1}=v_{1} \\
& \dot{z}_{2}=f_{2}\left(z_{1}, z_{2}, z_{3}, z_{4}\right)+z_{3} v_{1} \\
& \dot{z}_{3}=f_{3}\left(z_{1}, z_{2}, z_{3}, z_{4}\right)+z_{4} v_{1} \\
& \dot{z}_{4}=v_{2}
\end{aligned}
$$

Condition (3.12) or (3.16) is equivalent to $f_{2}$ being independent of $z_{4}$ :

$$
\frac{\partial f_{2}}{\partial z_{4}}=0
$$

and conditions (3.13) and (3.15), or (3.17) and (3.18), translate into:

$$
v_{1}+\frac{\partial f_{3}}{\partial z_{4}} \neq 0
$$

and

$$
\left(v_{1}+\frac{\partial f_{2}}{\partial z_{3}}, f_{3}-\frac{\partial f_{2}}{\partial z_{1}}-z_{3} \frac{\partial f_{2}}{\partial z_{2}}+z_{4} v_{1}\right) \neq(0,0)
$$

at the point under consideration. A pair of linearizing outputs is, for instance, given by $\left(z_{1}, z_{2}\right)$ at a point where $v_{1}+\frac{\partial f_{2}}{\partial z_{3}}$ does not vanish, and by $\left(z_{3}, z_{2}-z_{1} z_{3}\right)$ at a point where $f_{3}-\frac{\partial f_{2}}{\partial z_{1}}-z_{3} \frac{\partial f_{2}}{\partial z_{2}}+z_{4} v_{1}$ does not vanish.

Note that this theorem does not say anything about the situation around points $\bar{x}$ where

- either one of the distributions spanned by $\Delta_{2}, \mathcal{M}_{0}$ or $\mathcal{M}_{1}$ is singular,

- or they are regular, $\left(m_{0}, m_{1}\right) \neq(3,4)$ and the distribution spanned by $\Delta_{3}$ is singular,

- or $\left(m_{0}, m_{1}, \delta_{3}\right)=(2,2,3)$, the distribution spanned by $\Delta_{3}$-i.e. by $\left\{X_{1}, X_{2},\left[X_{0}, X_{1}\right],\left[X_{0}, X_{2}\right]\right\}$ since $\left(m_{0}, m_{1}\right)=(2,2)$ - has rank 3 and is integrable, but the distribution spanned by $\left\{X_{1}, X_{2},\left[X_{0},\left[X_{0}, X_{1}\right]\right],\left[X_{0}\right.\right.$, $\left.\left.\left[X_{0}, X_{2}\right]\right]\right\}$ is singular. 


\section{4. $(x, u)$-DYNAMIC LINEARIZABILITY}

\subsection{Problem statement}

Let us examine the situations in which theorem 3.1 concludes that there exist no pair of linearizing outputs depending on $x$ only for system (1.5), without ruling out existence of linearizing outputs depending on more variables $(u, \dot{u}, \ddot{u}, \ldots)$. This occurs

- in case 5 when (3.8) fails,

- in case 6 when $(3.12)$ fails,

- in case 6 when (3.12) is satisfied but (3.13) or (3.15) fails.

The first and third situations are singularities because (see theorem 3.1 ) in case $5,(3.8)$ is met on an open dense set, and in case 6 if (3.12) is satisfied, (3.13) or (3.15) are met on an open dense set. We will not study these two situations. The second situation does not correspond to a singularity since $X_{1}, X_{2},\left[X_{1}, X_{2}\right]$, and $\left[\tilde{X}, X_{0}\right]$ may very well be linearly independent (this is even generic) on an open set where $\left(m_{0}, m_{1}\right)=(3,4)$. We shall study this situation in the present section. We make one more non-singularity assumption: we rule out the points where the rank of $X_{1}, X_{2},\left[X_{1}, X_{2}\right],\left[\widetilde{X}, X_{0}\right]$ drops to 3 while being 4 at arbitrarily close points. Furthermore, the techniques that we will use require to be at a Brunovský-regular point (see definition 2.10). Brunovský-regularity translates into condition (4.4) below. It is clear that, on an open set where $\left(m_{0}, m_{1}\right)=(3,4)$ and $X_{1}, X_{2},\left[X_{1}, X_{2}\right]$, and $\left[\tilde{X}, X_{0}\right]$ are linearly independent, Brunovský-regular points form an open and dense set. Hence Brunovský-regularity is one more non-singularity assumption. It is needed for technical reasons, but the example in section 5 shows that it is not necessary. To sum up:

Rank assumptions made all over the present section: ( $\widetilde{X}$ is defined by (3.11))

$$
\begin{aligned}
\operatorname{rank}\left\{X_{1}, X_{2}\right\} & =2 \\
\operatorname{rank}\left\{X_{1}, X_{2},\left[X_{1}, X_{2}\right]\right\} & =3 \\
\operatorname{rank}\left\{X_{1}, X_{2},\left[X_{1}, X_{2}\right],\left[X_{1},\left[X_{1}, X_{2}\right]\right],\left[X_{2},\left[X_{1}, X_{2}\right]\right]\right\} & =4 \\
\operatorname{rank}\left\{X_{1}, X_{2},\left[X_{1}, X_{2}\right],\left[X_{0}, \widetilde{X}\right]\right\} & =4 \\
\operatorname{rank}\left\{X_{1}, X_{2},\left[X_{0}, X_{1}\right]-u_{2}\left[X_{1}, X_{2}\right],\left[X_{0}, X_{2}\right]+u_{1}\left[X_{1}, X_{2}\right]\right\} & =4 .
\end{aligned}
$$

From (4.1)-(4.2)-(4.3), we are in case 6 of theorem 3.1. (4.4) indicates that (3.12) does not hold, and hence from theorem 3.1, there exist no pair of linearizing outputs depending on $x$ only, i.e. system (1.5) is not $x$-dynamic linearizable. The purpose of this section 4 is to characterize the cases where system $(1.5)$ is $(x, u)$-dynamic linearizable, i.e. where there exists a pair of linearizing outputs depending on $x$ and $u$ (but not on $\dot{u}, \ddot{u}, \ldots$ ).

\subsection{Main Result}

Let us now proceed with some preparation for our characterization of $(x, u)$-dynamic linearizability. The following proposition provides a particular choice of $\omega_{1}$ and $\omega_{2}$ (basis of $\mathcal{H}_{2}$ ) such that the expressions of $d \omega_{1}$ and $\mathrm{d} \omega_{2}$ are convenient and "canonical".

Proposition 4.1. Let $(\bar{x}, \bar{u})$ be such that the rank conditions (4.1)-(4.2)(4.3)-(4.4)-(4.5) are satisfied. Let $\omega_{1}$ and $\omega_{2}$ to be two differential forms of Esaim: Cocv, June 1997, Vol. 2, Pr. 151-230 
degree 1, linear combinations of $\mathrm{d} x_{1}, \mathrm{~d} x_{2}, \mathrm{~d} x_{3}, \mathrm{~d} x_{4}$, such that none of these forms vanish at $(\bar{x}, \bar{u})$ and

$$
\begin{aligned}
& \omega_{1} \in\left\{X_{1}, X_{2},\left[X_{1}, X_{2}\right]\right\}^{\perp} \\
& \omega_{2} \in\left\{X_{1}, X_{2},\left[X_{0}+u_{1} X_{1}+u_{2} X_{2}, \widetilde{X}\right]\right\}^{\perp} .
\end{aligned}
$$

Then $\left\{\omega_{1}, \omega_{2}, \dot{\omega}_{1}, \dot{\omega}_{2}\right\}$ is a basis of $\operatorname{Span}\{\mathrm{d} x\}$ and there exist uniquely defined functions $\delta_{i, j}^{k}$ and $\gamma$ such that $\gamma$ and $\delta_{1,2}^{2}$ do not vanish at $(\bar{x}, \bar{u})$ and

$$
\begin{aligned}
\mathrm{d} \omega_{1} & \equiv \delta_{1,2}^{2} \omega_{2} \wedge \dot{\omega}_{2} \quad \operatorname{modulo} \omega_{1} \\
\mathrm{~d} \omega_{2} & \equiv \omega_{1} \wedge\left(\delta_{2,1}^{1} \dot{\omega}_{1}+\delta_{2,1}^{2} \dot{\omega}_{2}-\gamma \ddot{\omega}_{2}\right)+\gamma \dot{\omega}_{1} \wedge \dot{\omega}_{2} \quad \text { modulo } \omega_{2} \cdot(
\end{aligned}
$$

Note that it is clear from (4.6) that, in general, $\omega_{1}$ can be chosen so as to involve $x$ only, but $\omega_{2}$ involves $x$ and $u$, i.e. it is a linear combination of $\mathrm{d} x_{1}, \mathrm{~d} x_{2}, \mathrm{~d} x_{3}, \mathrm{~d} x_{4}$ with coefficients depending both on $x$ and $u$. The functions $\gamma$ and $\delta_{i, j}^{k}$ a priori depend on $x, u$ and a certain number of time-derivatives of $u$.

Proof. Suppose that $\omega_{1}$ and $\omega_{2}$ are chosen according to (4.6). Then (4.4) and (3.11) imply that the rank of $\left\{X_{1}, X_{2},\left[X_{1}, X_{2}\right],\left[X_{0}+u_{1} X_{1}+u_{2} X_{2}, \tilde{X}\right]\right\}$ is 4 , and hence that $\left\{\omega_{1}, \omega_{2}\right\}$ is a basis of the annihilator of $\left\{X_{1}, X_{2}\right\}$.

The fact that $\omega_{1}$ in the orthogonal of $\left\{X_{1}, X_{2},\left[X_{1}, X_{2}\right]\right\}$ implies that it is in the first derived system of the Pfaffian system $\left\{\omega_{1}, \omega_{2}\right\}$-see the Appendixand hence that

$$
\mathrm{d} \omega_{1}=\omega_{1} \wedge \Gamma_{1,1}+\omega_{2} \wedge \Gamma_{1,2}
$$

for some forms $\Gamma_{1,1}$ and $\Gamma_{1,2}$. Now the forms $\omega_{1}, \omega_{2}$ and $\Gamma_{1,2}$ must be linearly independent from (4.3), and then the Cartan characteristic system of $\left\{\omega_{1}\right\}$ is $\left\{\omega_{1}, \omega_{2}, \Gamma_{1,2}\right\}$ - see the Appendix (8.2)-, but, by definition of $\tilde{X}$, this characteristic system is the annihilator of $\widetilde{X}$, and a basis of the annihilator of $\widetilde{X}$ is $\left\{\omega_{1}, \omega_{2}, \dot{\omega}_{2}\right\}$ because, from $(2.10)$,

$$
0=\frac{\mathrm{d}}{\mathrm{dt}}\left\langle\omega_{2}, \tilde{X}\right\rangle=\left\langle\dot{\omega}_{2}, \tilde{X}\right\rangle+\left\langle\omega_{2},\left[X_{0}+u_{1} X_{1}+u_{2} X_{2}, \tilde{X}\right]\right\rangle
$$

and hence $\left\langle\dot{\omega}_{2}, \widetilde{X}\right\rangle$ is zero; this proves that $\Gamma_{1,2}$ must be a linear combination of $\omega_{1}, \omega_{2}$ and $\dot{\omega}_{2}$, which, substituted in (4.9), yields (4.7) with $\delta_{1,2}^{2}$ does not vanish because $\omega_{1}, \omega_{2}$ and $\Gamma_{1,2}$ are linearly independent.

On the other hand, $\left\{\omega_{1}, \omega_{2}\right\}$ is the annihilator of $\left\{X_{1}, X_{2}\right\}$ and therefore has a basis that can be written with the variable $x$ only; this implies - see (8.3) in the Appendix - that its characteristic system is at most Span $\{\mathrm{dx}\}$; since $\left\{\omega_{1}, \omega_{2}, \dot{\omega}_{1}, \dot{\omega}_{2}\right\}$ is a basis of $\operatorname{Span}\{\mathrm{dx}\}$, this implies

$$
\mathrm{d} \omega_{2}=\omega_{1} \wedge \Gamma_{2,1}+\omega_{2} \wedge \Gamma_{2,2}+\gamma \dot{\omega}_{1} \wedge \dot{\omega}_{2}
$$

for some forms $\Gamma_{2,1}$ and $\Gamma_{2,2}$. But we have seen above that $\left\{\omega_{1}, \omega_{2}, \dot{\omega}_{2}\right\}$ is the Cartan characteristic system of $\left\{\omega_{1}\right\}$. It is therefore completely integrable, and this implies that $\mathrm{d} \dot{\omega}_{2} \equiv 0$ modulo $\left\{\omega_{1}, \omega_{2}, \dot{\omega}_{2}\right\}$; but taking the time derivative of $(4.10)$ yields $\left.\mathrm{d} \dot{\omega}_{2} \equiv \dot{\omega}_{1} \wedge\left(\Gamma_{2,1}+\gamma \ddot{\omega}_{2}\right)\right)$ modulo $\left\{\omega_{1}, \omega_{2}, \dot{\omega}_{2}\right\}$; $\Gamma_{2,1} \equiv-\gamma \ddot{\omega}_{2}$, which does imply, together with (4.10), the relation (4.8).

We are now ready to state the theorem that characterizes $(x, u)$-linearizability. Its proof is given in section 7.2 . 
Theorem 4.2. Let $(\bar{x}, \bar{u})$ be a point where conditions (4.1) to (4.5) are met, and let the forms $\omega_{1}$ and $\omega_{2}$ be defined according to (4.6) and the functions $\delta_{2,1}^{1}$ and $\gamma$ be defined by (4.8). System (1.5) is $(x, u)$-dynamically linearizable at point $\overline{\mathcal{X}}=(\bar{x}, \bar{u}, \dot{\bar{u}}, \ldots)$ if and only if the function $\delta_{2,1}^{1}$-or equivalently the form of degree $5 \mathrm{~d} \omega_{2} \wedge \omega_{2} \wedge \dot{\omega}_{2} \wedge \ddot{\omega}_{2}$-does not vanish at $\overline{\mathcal{X}}$ and the first derived system of the Pfaffian system $\left\{\omega_{1}-\frac{2 \gamma}{\delta_{2,1}^{1}} \dot{\omega}_{2}, \omega_{2}\right\}$ has rank 1 and is integrable, i.e. there exists a function $\alpha$, defined on a neighborhood of $\overline{\mathcal{X}}$, such that

$$
\mathrm{d}\left(\omega_{1}+\alpha \omega_{2}-\frac{2 \gamma}{\delta_{2,1}^{1}} \dot{\omega}_{2}\right) \wedge\left(\omega_{1}+\alpha \omega_{2}-\frac{2 \gamma}{\delta_{2,1}^{1}} \dot{\omega}_{2}\right)=0 .
$$

When these conditions are met, all the possible pairs of linearizing outputs depending on $x$ and $u$ may be described as follows. Let $\Omega_{3}=\omega_{1}+\alpha \omega_{2}-\frac{2 \gamma}{\delta_{2,1}^{1}} \dot{\omega}_{2}$, and $\dot{\Omega}_{3}$ be the time-derivative of this differential form (i.e. its Lie derivative along the dynamics $F$ of the system). The Pfaffian system $\left\{\omega_{2}, \Omega_{3}, \dot{\Omega}_{3}\right\}$ is completely integrable. A pair of functions $\left(h_{1}, h_{2}\right)$ depending on $(x, u)$ is a pair of linearizing outputs if and only if $\left\{\mathrm{d} h_{1}, \mathrm{~d} h_{2}\right\} \subset\left\{\omega_{2}, \Omega_{3}, \dot{\Omega}_{3}\right\}$ with $\Omega_{3} \in\left\{\mathrm{d} h_{1}, \mathrm{~d} h_{2}\right\}$ and $\dot{\Omega}_{3} \notin\left\{\mathrm{d} h_{1}, \mathrm{~d} h_{2}\right\}$. A possible construction is as follows: since $\mathrm{d} \Omega_{3} \wedge \Omega_{3}=0$, take $h_{1}$ such that $\mathrm{d} h_{1}$ does not vanish and $\mathrm{d} h_{1}=k \Omega_{3}$ ( $k$ non-vanishing function); take for $h_{2}$ another first integral of $\left\{\omega_{2}, \Omega_{3}, \dot{\Omega}_{3}\right\}$ such that the coefficient of $\omega_{2}$ when expressing $\mathrm{d} h_{2}$ as a linear combination of $\omega_{2}, \Omega_{3}$ and $\dot{\Omega}_{3}$ does not vanish (i.e. the rank of $\left\{\mathrm{d} h_{2}, \mathrm{~d} h_{1}, \mathrm{~d} \dot{h}_{1}\right\}$ does not drop to 2$)$.

This theorem is stated in terms of the forms $\omega_{1}$ and $\omega_{2}$. These forms are only defined up to a non-vanishing multiplicative function by relation (4.6). However, the condition does not depend on the particular choice of $\omega_{1}$ and $\omega_{2}$. In a sense this is a consequence of the theorem itself since $(x, u)$-dynamic linearizability is clearly static feedback invariant and does not depend on the choice of $\omega_{1}$ and $\omega_{2}$, but the following proposition asserts that a priori these conditions are static feedback invariant.

Proposition 4.3. The conditions of theorem 4.2 are invariant by static feedback and do not depend on the particular choice of $\omega_{1}$ and $\omega_{2}$ in (4.6). Indeed the Pfaffian system $\left\{\omega_{2}, \omega_{1}-\frac{2 \gamma}{\delta_{2,1}^{1}} \dot{\omega}_{2}\right\}$ does not depend on this particular choice.

Proof. It can be checked from (4.8) that if one changes $\omega_{1}$ into $\lambda_{1} \omega_{1}$ and $\omega_{2}$ into $\lambda_{2} \omega_{2}$, where $\lambda_{1}$ and $\lambda_{2}$ are non-vanishing functions, then $\delta_{2,1}^{1}$ is changed into $\frac{\lambda_{2}}{\lambda_{1}^{2}} \delta_{2,1}^{1}$ and $\gamma$ into $\frac{1}{\lambda_{1}} \gamma$. This implies the proposition since (4.6) defines $\omega_{1}$ and $\omega_{2}$ up to a nonzero multiplicative function in a feedback invariant way.

Let us make a remark on "singular" points, i.e. points where the ranks considered in (4.1)-(4.2)-(4.3)-(4.4)-(4.5) are not constant. We do not study the situation at these points, in particular at points which are not Brunovskýregular, i.e. points where the rank in (4.5) drops. As illustrated by the example in section 5 , this singularity is usually not a singularity of $(x, u)$-dynamic linearization, but only of the proofs given here: the linearizing outputs are Esaim: Cocv, June 1997, Vol. 2, Pr. 151-230 
well defined at these points too, enjoy the property of being linearizing outputs. On the contrary, points where $\delta_{2,1}^{1}$, or the form $d \omega_{2} \wedge \omega_{2} \wedge \dot{\omega}_{2} \wedge \ddot{\omega}_{2}$, vanish are, according to the theorem, actual singularities of $(x, u)$-dynamic linearizability: in a domain where the rank assumptions (4.1)-(4.2)-(4.3)(4.4)-(4.5) hold, there exists no linearizing outputs function of $x$ and $u$ in the neighborhood of a point where $\delta_{2,1}^{1}$ vanishes. It is interesting, with this respect, to notice that, under the - generic- assumptions (4.1)-(4.2)(4.3)-(4.4)-(4.5), it is impossible to build an example where $(x, u)$-dynamic feedback linearization would be everywhere nonsingular since for any value of $x$ and $u$, there is a value of $\dot{u}$ where $\delta_{2,1}^{1}$ vanishes.

\subsection{How TO CHECK THE CONDITIONS}

We claim that the conditions of theorem 4.2 are completely explicit. Let us explain how to check them on a system (1.5) given by the expression of the vector fields $X_{0}, X_{1}$ and $X_{2}$ in some coordinates $x_{1}, x_{2}, x_{3}, x_{4}$ :

1. Compute $\omega_{1}$ and $\omega_{2}$ according to (4.6). This involves the computation of Lie brackets, and then finding the annihilator of some families of vectors, which in coordinates is common linear algebra (Gauss elimination).

2. Compute $\dot{\omega}_{1}, \dot{\omega}_{2}$ and $\ddot{\omega}_{2}$. The time-derivatives are Lie derivatives along the vector field $(2.5)$.

3. To compute $\delta_{2,1}^{1}$ and $\gamma$, use the following identities, consequence of (4.8):

$$
\begin{aligned}
\mathrm{d} \omega_{2} \wedge \omega_{2} \wedge \dot{\omega}_{2} \wedge \ddot{\omega}_{2} & =\delta_{2,1}^{1} \omega_{1} \wedge \dot{\omega}_{1} \wedge \omega_{2} \wedge \dot{\omega}_{2} \wedge \ddot{\omega}_{2} \\
\mathrm{~d} \omega_{2} \wedge \omega_{2} \wedge \dot{\omega}_{1} \wedge \dot{\omega}_{2} & =-\gamma \omega_{1} \wedge \ddot{\omega}_{2} \wedge \omega_{2} \wedge \dot{\omega}_{1} \wedge \dot{\omega}_{2} \\
& =-\gamma \omega_{1} \wedge \dot{\omega}_{1} \wedge \omega_{2} \wedge \dot{\omega}_{2} \wedge \ddot{\omega}_{2} \\
\mathrm{~d} \omega_{2} \wedge \omega_{1} \wedge \omega_{2} & =\gamma \dot{\omega}_{1} \wedge \dot{\omega}_{2} \wedge \omega_{1} \wedge \omega_{2} .
\end{aligned}
$$

Hence one may for instance compute the forms of degree $5 \mathrm{~d} \omega_{2} \wedge \omega_{2} \wedge \dot{\omega}_{2} \wedge$ $\ddot{\omega}_{2}$ and $\mathrm{d} \omega_{2} \wedge \omega_{2} \wedge \dot{\omega}_{1} \wedge \dot{\omega}_{2}$, check that the first one does not vanish, they appear to be of the form $\rho_{1} \mathrm{~d} x_{1} \wedge \mathrm{d} x_{2} \wedge \mathrm{d} x_{3} \wedge \mathrm{d} x_{4} \wedge \mathrm{d} u_{1}+\rho_{2} \mathrm{~d} x_{1} \wedge \mathrm{d} x_{2} \wedge \mathrm{d} x_{3} \wedge$ $\mathrm{d} x_{4} \wedge \mathrm{d} u_{2}$ and $\rho_{3} \mathrm{~d} x_{1} \wedge \mathrm{d} x_{2} \wedge \mathrm{d} x_{3} \wedge \mathrm{d} x_{4} \wedge \mathrm{d} u_{1}+\rho_{4} \mathrm{~d} x_{1} \wedge \mathrm{d} x_{2} \wedge \mathrm{d} x_{3} \wedge \mathrm{d} x_{4} \wedge \mathrm{d} u_{2}$ respectively, with $\rho_{1}, \rho_{2}, \rho_{3}$ and $\rho_{4}$ some functions of $x, u$ and $\dot{u}$, with $\rho_{1} \rho_{4}-\rho_{2} \rho_{3}=0$, then

$$
\frac{2 \gamma}{\delta_{2,1}^{1}}=-\frac{2 \rho_{3}}{\rho_{1}}=-\frac{2 \rho_{4}}{\rho_{2}} .
$$

4. The Pfaffian system $\left\{\omega_{1}-\frac{2 \gamma}{\delta_{2,1}^{1}} \dot{\omega}_{2}, \omega_{2}\right\}$ is then known.

5. Use usual procedure to compute its first derived system: the forms $\mathrm{d}\left(\omega_{1}-\frac{2 \gamma}{\delta_{2,1}^{1}} \dot{\omega}_{2}\right)$ and $\mathrm{d} \omega_{2}$ must be proportional modulo $\left\{\omega_{1}-\frac{2 \gamma}{\delta_{2,1}^{1}} \dot{\omega}_{2}, \omega_{2}\right\}$; if it is the case, this yields $\alpha$ such that $\mathrm{d}\left(\omega_{1}-\frac{2 \gamma}{\delta_{2,1}^{1}} \dot{\omega}_{2}+\alpha \omega_{2}\right)$ is zero $\operatorname{modulo}\left\{\omega_{1}-\frac{2 \gamma}{\delta_{2,1}^{1}} \dot{\omega}_{2}, \omega_{2}\right\}$.

6. Check whether $\mathrm{d}\left(\omega_{1}-\frac{2 \gamma}{\delta_{2,1}^{1}} \dot{\omega}_{2}+\alpha \omega_{2}\right)$ is also zero modulo $\omega_{1}-\frac{2 \gamma}{\delta_{2,1}^{1}} \dot{\omega}_{2}+$ $\alpha \omega_{2}$. 
Note that a small package written in Maple that makes the above computations, as well as these corresponding to theorem 3.1 , will soon be available from the author; it is described in [16].

\subsection{The result in particular coordinates}

Let us now give a "normal form" for the systems we are studying in this section, i.e. these meeting conditions (4.1)-(4.2)-(4.3)-(4.4)-(4.5). It basically consists, as in "case 6 " of theorem 3.1 , in taking some coordinates (they exist from (4.1)-(4.2)-(4.3)) in which the control distribution is in "Engel's normal form", and use a feedback to annihilate two components of the drift, then the coordinates are slightly changed to emphasize condition (4.4):

Proposition 4.4. If the rank conditions (4.1)-(4.2)-(4.3)-(4.4)-(4.5) hold around a point $(\bar{x}, \bar{u})$, there exists a system of coordinates around this point, and a static feedback defined around this point which give the following form to system (1.5):

$$
\left\{\begin{array}{l}
\dot{z}_{1}=v_{1} \\
\dot{z}_{2}=z_{4}+z_{3} v_{1} \\
\dot{z}_{3}=f\left(z_{1}, z_{2}, z_{3}, z_{4}\right)+g\left(z_{1}, z_{2}, z_{3}, z_{4}\right) v_{1} \\
\dot{z}_{4}=v_{2}
\end{array}\right.
$$

where

$$
\frac{\partial g}{\partial z_{4}}
$$

and

$$
\begin{aligned}
D_{1}= & \frac{\partial g}{\partial z_{4}}\left(v_{2}-f v_{1}\right)+z_{4} \frac{\partial g}{\partial z_{2}}+f \frac{\partial g}{\partial z_{3}} \\
& -\left(\frac{\partial f}{\partial z_{1}}+z_{3} \frac{\partial f}{\partial z_{2}}+g \frac{\partial f}{\partial z_{3}}+f \frac{\partial f}{\partial z_{4}}\right)
\end{aligned}
$$

do not vanish at $(\bar{x}, \bar{u})$.

Proof. From lemma 7.4 (section 7.1), using the feedback (7.30) yields the (3.20). Condition (4.4) implies that $\frac{\partial f_{2}}{\partial z_{4}}$ does not vanish. One may therefore take as new coordinates $\left(z_{1}, z_{2}, z_{3}, f_{2}\left(z_{1}, z_{2}, z_{3}, z_{4}\right)\right)$ instead of $\left(z_{1}, z_{2}, z_{3}, z_{4}\right)$, and this yields the normal form (4.13), changing also $v_{2}$. Relations (4.14) are simply a translation of (4.3) and (4.5).

Proposition 4.5. System (4.13) - which is system (1.5) written in appropriate coordinates - is $(x, u)$-dynamic linearizable around a point $\overline{\mathcal{X}}$ if and only if the functions $f$ and $g$ have, in a neighborhood of $\overline{\mathcal{X}}$, the form

$$
f=\frac{a_{0}+a_{1} z_{4}+a_{2} z_{4}^{2}}{c_{0}+c_{1} z_{4}} ; g=\frac{b_{0}+b_{1} z_{4}}{c_{0}+c_{1} z_{4}}
$$

where $a_{0}, a_{1}, a_{2}, b_{0}, b_{1}, c_{0}$ and $c_{1}$ are functions of $z_{1}, z_{2}, z_{3}$ only, which satisfy the following PDE:

$$
\mathrm{d} \Gamma \wedge \Gamma=0 \text { with } \Gamma=\left(b_{1}-z_{3} a_{2}\right) \mathrm{d} z_{1}+a_{2} \mathrm{~d} z_{2}-c_{1} \mathrm{~d} z_{3}
$$

and $\delta_{2,1}^{1}$ does not vanish at this point $\left(c_{0}+c_{1} z_{4}\right.$ should obviously not vanish either). 
REMARK 4.6. The system of PDEs (4.17) reads:

$$
\begin{aligned}
& z_{3}\left(c_{1} \frac{\partial a_{2}}{\partial z_{2}}-a_{2} \frac{\partial c_{1}}{\partial z_{2}}\right)+c_{1} \frac{\partial a_{2}}{\partial z_{1}}- a_{2} \frac{\partial c_{1}}{\partial z_{1}}+b_{1} \frac{\partial c_{1}}{\partial z_{2}}-c_{1} \frac{\partial b_{1}}{\partial z_{2}} \\
&-a_{2} \frac{\partial b_{1}}{\partial z_{3}}+b_{1} \frac{\partial a_{2}}{\partial z_{3}}+a_{2}{ }^{2}=0 .
\end{aligned}
$$

REMARK 4.7. There is an explicit formula for $\delta_{2,1}^{1}$ using the $a_{i}, b_{i}$ and $c_{i}$ but it is quite long, and does not really matter here.

This proposition gives a simple way to check whether the system is $(x, u)$ dynamic linearizable provided one has found coordinates where it is in the normal form (4.13) - of course finding these coordinates involves solving some linear PDEs, so that the really explicit test is given by theorem 4.2 which only involves some differentiations, and some algebraic manipulations-. Actually, the coordinates in which a given system meeting conditions (4.1)-(4.2)-(4.3)-(4.4)-(4.5) is in the form (4.13) are not unique, and the expression of $f$ and $g$, for the same system, may depend on the choice of coordinates, among all these that yield a form like (4.13)). Naturally, the fact that these $f$ and $g$ meet or not the conditions of the proposition does not depend on this choice. It however raises the question of finding, among all the coordinates that produce a normal form like (4.13), these which produce the "simplest" $f$ and $g$. Let us give an answer only for the special case when the conditions of the proposition are met (i.e. in the $(x, u)$-linearizable case). It is obvious that if $f$ and $g$ are affine in $z_{4}$ (special case of (4.16): $a_{2}=c_{1}=0, c_{0}=1$ ), the $\operatorname{PDE}(4.11)$ is met, because $\Gamma$ is simply $b_{1} \mathrm{~d} z_{1}$; it turns out that the converse is true: if $f$ and $g$ are not affine, but of the form (4.16) with $a_{2} \neq 0$ or $c_{1} \neq 0$, and with the PDE (4.11), then some "better" coordinates may be found, in which $f$ and $g$ are affine in the fourth coordinate:

Proposition 4.8. There exists coordinates where the system, after a static feedback transformation, is in the form (4.13) with $f$ and $g$ satisfying the conditions of proposition 4.5, if and only if there is another set of coordinates $\left(\zeta_{1}, \zeta_{2}, \zeta_{3}, \zeta_{4}\right)$, and another static feedback transformation which yields a normal form (4.13) with $f$ and $g$ affine with respect to the fourth coordinate:

$$
\left\{\begin{aligned}
\dot{\zeta}_{1}= & w_{1} \\
\dot{\zeta}_{2}= & \zeta_{4}+\zeta_{3} w_{1} \\
\dot{\zeta}_{3}= & p_{0}\left(\zeta_{1}, \zeta_{2}, \zeta_{3}\right)+\zeta_{4} p_{1}\left(\zeta_{1}, \zeta_{2}, \zeta_{3}\right) \\
& \quad+\left(q_{0}\left(\zeta_{1}, \zeta_{2}, \zeta_{3}\right)+\zeta_{4} q_{1}\left(\zeta_{1}, \zeta_{2}, \zeta_{3}\right)\right) w_{1} \\
\dot{\zeta}_{4}= & w_{2}
\end{aligned}\right.
$$

and $\delta_{2,1}^{1}$ does not vanish if and only if the following quantity does not vanish:

$$
\begin{aligned}
& q_{1} \dot{w}_{1}+w_{1}\left(p_{1}+w_{1} q_{1}\right)^{2}+w_{1} \frac{\partial}{\partial \zeta_{1}}\left(p_{1}+w_{1} q_{1}\right) \\
& \quad-\frac{\partial}{\partial \zeta_{2}}\left[\left(p_{0}+w_{1} q_{0}\right)-\zeta_{3} w_{1}\left(p_{1}+w_{1} q_{1}\right)\right]-\left(p_{1}+w_{1} q_{1}\right)^{2} \frac{\partial}{\partial \zeta_{3}} \frac{p_{0}+w_{1} q_{0}}{p_{1}+w_{1} q_{1}}
\end{aligned}
$$

In these coordinates, a pair of linearizing outputs is given by $h_{1}=\zeta_{1}, h_{2}=$ $\zeta_{3}-\left(p_{1}-w_{1} q_{1}\right) \zeta_{2}$. 
Proof. The expression (4.20) is obtained by computing $\mathrm{d} \omega_{2} \wedge \omega_{2} \wedge \dot{\omega}_{2} \wedge \ddot{\omega}_{2}$ and checking that it vanishes if and only if $(4.20)$ vanishes, at least at points where (4.5) holds, i.e. where $\omega_{1} \wedge \omega_{2} \wedge \dot{\omega}_{2} \neq 0$. This is left to the reader. Use the simplest choice:

$$
\begin{aligned}
& \omega_{1}=\mathrm{d} \zeta_{2}-\zeta_{3} \mathrm{~d} \zeta_{1} \\
& \omega_{2}=\mathrm{d} \zeta_{3}-q_{1}\left(\zeta_{1}, \zeta_{2}, \zeta_{3}\right) \mathrm{d} \zeta_{1}-\left(p_{1}\left(\zeta_{1}, \zeta_{2}, \zeta_{3}\right)+w_{1} q_{1}\left(\zeta_{1}, \zeta_{2}, \zeta_{3}\right)\right) \omega_{1} .
\end{aligned}
$$

The "if" part of the proposition is obvious because, as noticed just above the proposition, (4.19) is a particular case of (4.13)-(4.11), and (4.20) ensures that $\delta_{2,1}^{1} \neq 0$. Let us prove the "only if" part. We suppose that the conditions of proposition 4.5 hold, and we build an invertible transformation $\left(z_{1}, z_{2}, z_{3}, z_{4}\right) \mapsto\left(\zeta_{1}, \zeta_{2}, \zeta_{3}, \zeta_{4}\right)$, and an invertible static feedback transformation $\left(z_{1}, z_{2}, z_{3}, z_{4}, v_{1}, v_{2}\right) \mapsto\left(z_{1}, z_{2}, z_{3}, z_{4}, w_{1}, w_{2}\right)$, that transforms (4.13) into (4.19). Condition (4.17) implies that there exists a function $\psi_{1}\left(z_{1}, z_{2}, z_{3}\right)$ and a non-vanishing function $k\left(z_{1}, z_{2}, z_{3}\right)$ such that

$$
\mathrm{d} \psi_{1}=k \Gamma \text {. }
$$

Now, $\omega_{1}$ may be chosen $\omega_{1}=\mathrm{d} z_{2}-z_{3} \mathrm{~d} z_{1}$ and then $\Gamma$ defined in (4.17) is also equal to: $\Gamma=b_{1} \mathrm{~d} z_{1}+a_{2} \omega_{1}-c_{1} \mathrm{~d} z_{3}$. Since the rank of $\left\{\mathrm{d} z_{1}, \mathrm{~d} z_{3}, \omega_{1}\right\}$ is 3 and $b_{1}$ and $c_{1}$ do not vanish simultaneously (this would cause $\frac{\partial g}{\partial z_{4}}$ to vanish), the rank of $\left\{\omega_{1}, \Gamma\right\}$ is locally constant, equal to 2 , and this Pfaffian system is therefore completely integrable, because these two forms involve only three variables $\left(z_{1}, z_{2}, z_{3}\right)$; hence there exists three functions $\psi_{2}, k^{\prime}, k^{\prime \prime}$, such that

$$
\mathrm{d} \psi_{2}=k^{\prime} \omega_{1}+k^{\prime \prime} \Gamma, \quad k^{\prime} \neq 0 .
$$

Let us then define

$$
\begin{aligned}
w_{1} & =\dot{\psi}_{1}=k\left\langle\Gamma, X_{0}+u_{1} X_{1}+u_{2} X_{2}\right\rangle \\
& =k \frac{\left(c_{0} b_{1}-c_{1} b_{0}\right) v_{1}-c_{1} a_{0}+\left(c_{0} a_{2}-a_{1} c_{1}\right) z_{4}}{c_{0}+c_{1} z_{4}}
\end{aligned}
$$

From this equation, one may express $v_{1}$ as a function of $w_{1}$. Substituting $v_{1}$ for this expression in (4.13)-(4.16), one obtains the following expressions for $\dot{z}_{1}, \dot{z}_{2}, \dot{z}_{3}$, which are now linear with respect to $z_{4}$ :

$$
\begin{aligned}
& \dot{z}_{1}=\frac{1}{c_{0} b_{1}-c_{1} b_{0}}\left(\frac{c_{0}+c_{1} z_{4}}{k} w_{1}+c_{1} a_{0}+\left(a_{1} c_{1}-c_{0} a_{2}\right) z_{4}\right) \\
& \dot{z}_{2}=\frac{1}{c_{0} b_{1}-c_{1} b_{0}}\left(\frac{z_{3}}{k}\left(c_{0}+c_{1} z_{4}\right) w_{1}+z_{3} c_{1} a_{0}+\right. \\
& \left.\left(c_{0} b_{1}-c_{1} b_{0}+a_{1} c_{1}-c_{0} a_{2}\right) z_{4}\right) \\
& \dot{z}_{3}=\frac{b_{0}+b_{1} z_{4}}{k\left(c_{0} b_{1}-c_{1} b_{0}\right)} w_{1}+a_{0} b_{1}+\left(a_{1} b_{1}-a_{2} b_{0}\right) z_{4} .
\end{aligned}
$$

Let us then define

$$
\begin{aligned}
\zeta_{1} & =\psi_{1}\left(z_{1}, z_{2}, z_{3}\right) \\
\zeta_{2} & =\psi_{2}\left(z_{1}, z_{2}, z_{3}\right) \\
\zeta_{3} & =\frac{k^{\prime \prime}\left(z_{1}, z_{2}, z_{3}\right)}{k\left(z_{1}, z_{2}, z_{3}\right)} \\
\zeta_{4} & =k^{\prime}\left(z_{1}, z_{2}, z_{3}\right) z_{4} .
\end{aligned}
$$


Let us see that in these coordinates, and with $w_{1}$ given by (4.23), we have (4.19):

$-\dot{\zeta}_{1}=w_{1}$ is a consequence of (4.27) and (4.23).

- From (4.28), $\dot{\zeta}_{2}=\left\langle\mathrm{d} \psi_{2}, X_{0}+u_{1} X_{1}+u_{2} X_{2}\right\rangle$, which is also equal, from (4.23) and (4.22), to $\frac{k^{\prime \prime}}{k} w_{1}+k^{\prime}\left\langle\omega_{1}, X_{0}+u_{1} X_{1}+u_{2} X_{2}\right\rangle$, which, since $\left\langle\omega_{1}, X_{0}+u_{1} X_{1}+u_{2} X_{2}\right\rangle=z_{4}$, and considering (4.29) and (4.30), yields $\dot{\zeta}_{2}=\zeta_{4}+\zeta_{3} w_{1}$.

- In the expressions for $\dot{z}_{1}, \dot{z}_{2}$ and $\dot{z}_{3}$ given by (4.24), (4.25) and (4.26), all the functions of $\left(z_{1}, z_{2}, z_{3}\right)$ may be expressed as functions of $\left(\zeta_{1}, \zeta_{2}, \zeta_{3}\right)$, and $z_{4}$ may be substituted for $\frac{\zeta_{4}}{k^{\prime}}$ (see $(4.30)$ ); therefore, $\dot{z}_{1}, \dot{z}_{2}$ and $\dot{z}_{3}$ are polynomials in $\zeta_{4}$ and $w_{1}$ with coefficients function of $\left(\zeta_{1}, \zeta_{2}, \zeta_{3}\right.$ with one term of degree zero, one term of degree 1 in $\zeta_{4}$, one term of degree 1 in $w_{1}$ and one term of degree 2 in $\zeta_{4} w_{1}$; since $\zeta_{3}$ is a function of $\left(z_{1}, z_{2}, z_{3}\right), \dot{\zeta}_{3}$ is also such a polynomial, which allows one to define functions $p_{0}, p_{1}, q_{0}$ and $q_{1}$ such that $\dot{\zeta}_{3}$ is as in (4.19).

$-\dot{\zeta}_{4}$ is equal to $k^{\prime}\left(\zeta_{1}, \zeta_{2}, \zeta_{3}\right) v_{2}$ plus some terms which depend only on $\zeta_{1}, \zeta_{2}$, $\zeta_{3}, \zeta_{4}$ and $v_{1}$. Since $k^{\prime}$ does not vanish, calling all this expression $w_{2}$ defines a nonsingular feedback that yields the required form.

\section{AN EXAMPLE}

Let us consider the following system, which is given as example 2 in [7]:

$$
\begin{aligned}
& \dot{x}_{1}=x_{2}+x_{3} u_{2} \\
& \dot{x}_{2}=x_{3}+x_{1} u_{2} \\
& \dot{x}_{3}=u_{1}+x_{2} u_{2} \\
& \dot{x}_{4}=u_{2} .
\end{aligned}
$$

The transformation $z_{1}=x_{4}, z_{2}=x_{2}, z_{3}=x_{1}, z_{4}=x_{3}, v_{1}=u_{2}, v_{2}=u_{1}+$ $x_{2} u_{2}$ puts it into the form (4.19), known to be $(x, u)$-dynamic linearizable. Let us however follow the general method. We have:

$$
\begin{gathered}
\tilde{X}=X_{1}=\frac{\partial}{\partial x_{3}}, X_{2}=x_{3} \frac{\partial}{\partial x_{1}}+x_{1} \frac{\partial}{\partial x_{2}}+x_{2} \frac{\partial}{\partial x_{3}}+\frac{\partial}{\partial x_{4}}, \\
{\left[X_{1}, X_{2}\right]=\frac{\partial}{\partial x_{1}},\left[X_{0}, \tilde{X}\right]=-\frac{\partial}{\partial x_{2}},} \\
{\left[X_{0}+u_{1} X_{1}+u_{2} X_{2}, \tilde{X}\right]=-\frac{\partial}{\partial x_{2}}-u_{2} \frac{\partial}{\partial x_{1}} .}
\end{gathered}
$$

Brunovský-regular points are points where (4.5) holds, i.e. points where

$$
x_{1}-u_{1} \neq 0 \text {. }
$$

The simplest choice for $\omega_{1}$ and $\omega_{2}$ is (see (4.6)):

$$
\begin{aligned}
& \omega_{1}=\mathrm{d} x_{2}-x_{1} \mathrm{~d} x_{4}, \\
& \omega_{2}=\mathrm{d} x_{1}-u_{2} \mathrm{~d} x_{2}+\left(u_{2} x_{1}-x_{3}\right) \mathrm{d} x_{4} .
\end{aligned}
$$

By expressing $\mathrm{d} \omega_{2}=-\mathrm{d} u_{2} \wedge \mathrm{d} x_{2}+\mathrm{d}\left(u_{2} x_{1}-x_{3}\right) \wedge \mathrm{d} x_{4}$ in the basis $\left\{\omega_{1}, \omega_{2}, \dot{\omega}_{1}\right.$, $\left.\dot{\omega}_{2}, \ddot{\omega}_{2}\right\}$ (at points where (5.2) holds), with

$$
\begin{aligned}
& \dot{\omega}_{1}=\mathrm{d} x_{3}+u_{2} \mathrm{~d} x_{1}-\left(x_{2}+u_{2} x_{3}\right) \mathrm{d} x_{4} \\
& \dot{\omega}_{2}=-u_{2}^{2} \mathrm{~d} x_{1}+\left(1-\dot{u}_{2}\right) \mathrm{d} x_{2}+\left(-u_{1}+x_{3} u_{2}^{2}+x_{1} \dot{u}_{2}\right) \mathrm{d} x_{4} \cdot \\
& \ddot{\omega}_{2}=\left(x_{1}-u_{1}\right) \mathrm{d} u_{2}+(\cdots) \mathrm{d} x_{1}+(\cdots) \mathrm{d} x_{2}+(\cdots) \mathrm{d} x_{3}+(\cdots) \mathrm{d} x_{4},
\end{aligned}
$$


one obtains an expression like (4.8) with:

$$
\delta_{2,1}^{1}=\frac{2\left(\dot{u}_{2}+u_{2}^{3}-1\right)}{x_{1}-u_{1}} \quad \text { and } \quad \gamma=-\frac{1}{x_{1}-u_{1}},
$$

so that $\delta_{2,1}^{1} \neq 0$ is equivalent to $\dot{u}_{2}+u_{2}^{3}-1 \neq 0$. Then the form $\omega_{3}=$ $\omega_{1}-\frac{2 \gamma}{\delta_{2,1}^{1}} \dot{\omega}_{2}$ may be explicitly computed. $d \omega_{2} \wedge \omega_{2} \wedge \omega_{3}$ and $d \omega_{3} \wedge \omega_{2} \wedge \omega_{3}$ are collinear:

$$
\mathrm{d} \omega_{3} \wedge \omega_{2} \wedge \omega_{3}=-\alpha \mathrm{d} \omega_{2} \wedge \omega_{2} \wedge \omega_{3} \quad \text { with } \quad \alpha=\frac{u_{2}^{2}}{\dot{u}_{2}+u_{2}^{3}-1} .
$$

A basis of the derived system of $\left\{\omega_{2}, \omega_{1}-\frac{2 \gamma}{\delta_{2,1}^{1}} \dot{\omega}_{2}\right\}$ is therefore

$$
\Omega_{3}=\omega_{1}-\frac{2 \gamma}{\delta_{2,1}^{1}} \dot{\omega}_{2}+\alpha \omega_{2}=\frac{x_{1}-u_{1}}{\dot{u}_{2}+u_{2}^{3}-1} \mathrm{~d} x_{4} .
$$

It is obviously integrable, condition (4.11) of theorem 4.2 is satisfied, hence the system is $(x, u)$-dynamic linearizable at points where $\delta_{2,1}^{1}$ does not vanish. Since $x_{4}$ is a first integral of $\left\{\Omega_{3}\right\}$, and a basis (at points where (5.1) holds) for the Pfaffian system $\left\{\omega_{2}, \Omega_{3}, \dot{\Omega}_{3}\right\}$ is $\left\{\mathrm{d} x_{1}-u_{2} \mathrm{~d} x_{2}, \mathrm{~d} x_{4}, \mathrm{~d} u_{2}\right\}$-it is indeed integrable, and three independent first integrals are $x_{4}, u_{2}$ and $x_{1}-x_{2} u_{2}$, theorem 4.2 implies that two functions $\left(h_{1}(x, u), h_{2}(x, u)\right)$ form a pair of linearizing outputs if and only if $h_{1}$ and $h_{2}$ are two independent functions of $x_{4}, u_{2}$ and $x_{1}-x_{2} u_{2}$ such that $\mathrm{d} h_{1}, \mathrm{~d} h_{2}, \mathrm{~d} u_{2}$ are independent but $\mathrm{d} x_{4}$ is a linear combination of $\mathrm{d} h_{1}$ and $\mathrm{d} h_{2}$. The simplest choice is

$$
h_{1}=x_{4} \quad \text { and } \quad h_{2}=x_{1}-u_{2} x_{2} .
$$

Let us illustrate on this example the invertible transformations on pairs of differential forms introduced in section 2.5 (following $[2,1]$ ). The functions $h_{1}$ and $h_{2}$ given by (5.8) are related to the forms $\omega_{1}$ and $\omega_{2}$ defining the “infinitesimal Brunovský form" by:

$$
\begin{aligned}
\left(\begin{array}{l}
\mathrm{d} h_{1} \\
\mathrm{~d} h_{2}
\end{array}\right)= & \left(\begin{array}{cc}
1 & -x_{2} \frac{\mathrm{d}}{\mathrm{dt}}-\left(u_{2} x_{1}-x_{3}\right) \\
0 & 1
\end{array}\right)\left(\begin{array}{cc}
1 & 0 \\
0 & \frac{\dot{u}_{2}+u_{2}^{3}-1}{x_{1}-u_{1}}
\end{array}\right) \\
& \times\left(\begin{array}{cc}
1 & 0 \\
-b \frac{\mathrm{d}}{\mathrm{dt}} & 1
\end{array}\right)\left(\begin{array}{ll}
0 & 1 \\
1 & \alpha
\end{array}\right)\left(\begin{array}{l}
\omega_{1} \\
\omega_{2}
\end{array}\right)
\end{aligned}
$$

with $b=\frac{2 \gamma}{\delta_{2,1}^{1}}$ (this may be re-arranged into an expression like (7.54) with some scalar function $a$ and matrix function $J_{1}$ ). Indeed from Proposition 2.6 and 2.9 , and since $\left(\omega_{1}, \omega_{2}\right)$ is a linearizing Pfaffian system and the matrices in the right-hand side of (5.9) are all invertible, this is enough to prove that $\left(h_{1}, h_{2}\right)$ is a pair of linearizing outputs at Brunovský-regular points. Note that the expressions in (5.9) are indeed singular at "Brunovský-singular points" - points that are not Brunovský-regular - so that the ideas based on the infinitesimal Brunovský form fail at these points, while linearizing outputs $h_{1}$ and $h_{2}$ may obviously be continued at these points, and it may be checked directly that they continue to be linearizing outputs at these Esaim: Cocv, June 1997, Vol. 2, Pr. 151-230 
points; indeed, since

$$
\begin{aligned}
\dot{h}_{1} & =u_{2}, \\
\ddot{h}_{1} & =\dot{u}_{2}, \\
h_{1}^{(3)} & =\ddot{u}_{2}, \\
\dot{h}_{2} & =x_{2}-x_{1} u_{2}^{2}-x_{2} \dot{u}_{2}, \\
\ddot{h}_{2} & =x_{3}+x_{1} u_{2}-x_{2} u_{2}^{2}-x_{3} u_{2}^{3}-\left(x_{3}+x_{1} u_{2}\right) \dot{u}_{2}-x_{2} \ddot{u}_{2},
\end{aligned}
$$

one may solve for $x_{1}, x_{2}, x_{3}, x_{4}, u_{2}, \dot{u}_{2}$ and $\ddot{u}_{2}$ in (5.8)-(5.10) and express them as (rational) functions of $h_{1}, \dot{h}_{1}, \ddot{h}_{1}, h_{1}^{(3)}, h_{2}, \dot{h}_{2}, \ddot{h}_{2}$ at all points where $\dot{u}_{2}+u_{2}^{3}-1 \neq 0$. It is clear on this example that the requirement of Brunovský-regularity is purely technical, and the singularities of dynamic feedback linearization are not related to the singularities of the "infinitesimal Brunovsky form". The singularity $\delta_{2,1}^{1}=0$, on the other hand is really a singularity of $(x, u)$-dynamic linearization.

The conclusion for this system is:

- It is not $x$-dynamic linearizable at any point, as a consequence of theorem 3.1, case 6 .

- It is $(x, u)$-dynamic linearizable at all points where $\dot{u}_{2}+u_{2}^{3}-1 \neq 0$.

This is a consequence of theorem 4.2 at points where $x_{1}-u_{1} \neq 0$. At points where $\dot{u}_{2}+u_{2}^{3}-1 \neq 0$ and $x_{1}-u_{1}=0$, it is not a consequence of theorem 4.2, but is clear from (5.10).

- It is not $(x, u)$-dynamic linearizable at points where $\dot{u}_{2}+u_{2}^{3}-1=0$. This is a consequence of theorem 4.2 at points where $x_{1}-u_{1} \neq 0$. At points where $x_{1}-u_{1}=\dot{u}_{2}+u_{2}^{3}-1=0$, this is not a consequence of theorem 4.2 , but may be proved as follows. Suppose that there is a pair of linearizing outputs $\left(h_{1}, h_{2}\right)$ in an open neighborhood of such a point. Points where $x_{1}-u_{1} \neq 0$ are dense on this neighborhood, and $\left(h_{1}, h_{2}\right)$ is still a pair of linearizing outputs at these points (if the neighborhood is small enough). Hence (see above) $h_{1}$ and $h_{2}$ are functions of $x_{4}, u_{2}$ and $x_{1}-u_{2} x_{2}: h_{i}\left(x_{1}, x_{2}, x_{3}, x_{4}, u_{1}, u_{2}\right)=\chi_{i}\left(x_{4}, u_{2}, x_{1}-u_{2} x_{2}\right)$. Because the rank of $\mathrm{d} x_{4}, \mathrm{~d} u_{2} \mathrm{~d}\left(x_{1}-u_{2} x_{2}\right)$ is 3 , the smooth functions $\chi_{i}$ are unique and may be prolonged at the point under consideration (where $u_{1}-x_{1}$ vanishes). Computing the time-derivatives of the functions $h_{i}$ from these identities, it can be seen that their partial derivative with respect to $x_{2}$ all vanish at points where $\dot{u}_{2}+u_{2}^{3}-1=0$. This prevents $x_{2}$ from being, around such a point, a smooth function of $h_{1}, h_{2}, \dot{h}_{1}, \dot{h}_{2}, \ddot{h}_{1}, \ddot{h}_{2}, \ldots$, and hence $\left(h_{1}, h_{2}\right)$ from being a pair of linearizing outputs at these points.

Note that the singularity $\dot{u}_{2}+u_{2}^{3}-1=0$ does not correspond to a singularity of the linear approximation. Consider for instance the solution

$$
\begin{array}{r}
u_{1}(t)=-1, \quad u_{2}(t)=1, \quad x_{1}(t)=x_{2}(t)=1, x_{3}(t)=-1, x_{4}(t)=t . \\
\text { Esaim: Cocv, JUne 1997, Vol. 2, Pr. } 151-230
\end{array}
$$


Clearly $\dot{u}_{2}+u_{2}^{3}-1$ is zero along this solution, while the linear approximation $\delta \dot{x}=A \delta x+B \delta u$, with

$$
A=\left(\begin{array}{llll}
0 & 1 & 1 & 0 \\
1 & 0 & 1 & 0 \\
0 & 1 & 0 & 0 \\
0 & 0 & 0 & 0
\end{array}\right) \quad \text { and } B=\left(\begin{array}{cc}
0 & -1 \\
0 & 1 \\
1 & 1 \\
0 & 1
\end{array}\right)
$$

is controllable. An example where this occurs at an equilibrium instead of a nontrivial solution is obtained by replacing $x_{2}$ with $x_{4}$ in $\dot{x}_{1}$, the singularity $\delta_{2,1}^{1}=0$ then occurs when $\dot{u}_{2}+u_{2}^{3}=0$ while the linear approximation at $(x, u)=(0,0)$ is controllable.

\section{NON-AFFINE SYSTEMS IN $\mathbb{R}^{3}$}

Consider a system

$$
\dot{\xi}=f\left(\xi, w_{1}, w_{2}\right)
$$

where $\xi$ lives in $\mathbb{R}^{3}$. A system of the form (1.5) can always be brought to this form at a point where one of the control vector fields does not vanish by finding coordinates in which this control vector field is the first coordinate vector field, dropping the corresponding control and taking this first coordinate as a new control. The converse is not correct in general.

However a necessary condition for feedback linearization, that can be found in [22] or in [24] implies that if system (6.1) linearizable by dynamic feedback (even in a more general sense than endogenous), it has a dynamic extension of dimension 4 which is affine in the control. The following proposition is a consequence of theorem 1 in [22], except the regularity of $\gamma$, but this is automatic if one wants the linearizing outputs to be smooth:

Proposition 6.1 ([22]). At a point $\left(\bar{\xi}, \bar{w}_{1}, \bar{w}_{2}\right)$ where rank $\left\{\frac{\partial f}{\partial w_{1}}, \frac{\partial f}{\partial w_{2}}\right\}$ is 2, a necessary condition for system (6.1) to be dynamic feedback linearizable is that there exist, locally around $\left(\bar{\xi}, \bar{w}_{1}, \bar{w}_{2}\right)$, a static feedback transformation $\left(w_{1}, w_{2}\right)=\gamma\left(\xi, v_{1}, v_{2}\right)$ such that $f\left(\xi, \gamma\left(\xi, v_{1}, v_{2}\right)\right)$ be affine with respect to $v_{1}$ : $f\left(\xi, \gamma\left(\xi, v_{1}, v_{2}\right)\right)=a\left(\xi, v_{2}\right)+v_{1} b\left(\xi, v_{2}\right)$.

In the case of system (6.1), an explicit condition for existence of this static feedback transformation may be given, but this is outside the scope of the present paper. It is clear that the necessary condition for dynamic linearization given in proposition 6.1 is exactly the condition needed to transform system (6.1) into an affine 4-dimensional system. This is summed up in the following result, which allows one to apply to 3-dimensional non-affine systems (6.1) all the results obtained in the previous sections for 4-dimensional affine systems.

Proposition 6.2. At a point $\left(\bar{\xi}, \bar{w}_{1}, \bar{w}_{2}\right)$ where $\operatorname{rank}\left\{\frac{\partial f}{\partial w_{1}}, \frac{\partial f}{\partial w_{1}}\right\}$ is 2, either system (6.1) is not dynamic feedback linearizable or one may construct a Esaim: Cocv, June 1997, Vol. 2, Pr. 151-230 
static feedback transformation $\left(w_{1}, w_{2}\right)=\gamma\left(\xi, v_{1}, v_{2}\right)$ such that dynamic feedback linearization of (6.1) is equivalent to dynamic feedback linearization of

$$
\begin{aligned}
\left(\begin{array}{c}
\dot{x}_{1} \\
\dot{x}_{2} \\
\dot{x}_{3}
\end{array}\right) & =a\left(x_{1}, x_{2}, x_{3}, x_{4}\right)+u_{1} b\left(x_{1}, x_{2}, x_{3}, x_{4}\right) \\
\dot{x}_{4} & =u_{2} .
\end{aligned}
$$

\section{THE PROOFS}

All over these proofs, some known facts about Pfaffian systems (derived systems, characteristic system ...) are used. They are briefly recalled in the Appendix.

\subsection{Proof of TheOrem 3.1}

CASE $1\left(m_{0}=m_{1}=2, \delta_{3}=2\right) \cdot m_{0}=2$ means that the distribution spanned by the control vector fields $X_{1}$ and $X_{2}$ is involutive. Frobenius theorem yields a set of coordinates $\left(z_{1}, z_{2}, z_{3}, z_{4}\right)$ such that $\left\{\frac{\partial}{\partial z_{3}}, \frac{\partial}{\partial z_{4}}\right\}$ is a basis of this distribution, then

$$
\begin{aligned}
& v_{1}=L_{X_{0}} z_{3}+u_{1} L_{X_{1}} z_{3}+u_{2} L_{X_{2}} z_{3} \\
& v_{2}=L_{X_{0}} z_{4}+u_{1} L_{X_{1}} z_{4}+u_{2} L_{X_{2}} z_{4}
\end{aligned}
$$

is a nonsingular static feed back because $X_{1}$ and $X_{2}$ are independent at point $\bar{x}$. System (1.5) reads, in the above coordinates as

$$
\begin{array}{ll}
\dot{z}_{1}=a_{1}\left(z_{1}, z_{2}, z_{3}, z_{4}\right) & \dot{z}_{3}=v_{1} \\
\dot{z}_{2}=a_{2}\left(z_{1}, z_{2}, z_{3}, z_{4}\right) & \dot{z}_{4}=v_{2} .
\end{array}
$$

$\Delta_{3}$ is then spanned by $\frac{\partial}{\partial z_{3}}, \frac{\partial}{\partial z_{4}}, \frac{\partial a_{1}}{\partial z_{3}} \frac{\partial}{\partial z_{1}}+\frac{\partial a_{2}}{\partial z_{3}} \frac{\partial}{\partial z_{2}}$ and $\frac{\partial a_{1}}{\partial z_{4}} \frac{\partial}{\partial z_{1}}+\frac{\partial a_{2}}{\partial z_{4}} \frac{\partial}{\partial z_{2}} . \delta_{3}=2$ implies that $a_{1}$ and $a_{2}$ do not depend on $z_{3}$ and $z_{4}$. This yields (3.3).

CASE 2.A $\left(m_{0}=m_{1}=2, \delta_{3}=3\right)$. Since $\left\{X_{1}, X_{2}\right\}$ is integrable of rank 2 , there exists two independent functions constant along $X_{1}$ and $X_{2}$, and one of them at least has either its Lie derivative along $\left[X_{0}, X_{1}\right]$ or its Lie derivative along $\left[X_{0}, X_{2}\right]$ that does not vanish at $\bar{x}$ because if not the rank of $\Delta_{3}$ would drop to two; let $z_{2}$ be this one, and $z_{1}$ be the other one, and define $z_{3}=L_{X_{0}} z_{2} . L_{X_{1}} z_{3}$ or $L_{X_{2}} z_{3}$ does not vanish at $\bar{x}$ (because they are equal to $L_{\left[X_{1}, X_{0}\right]} z_{2}$ and $\left.L_{\left[X_{2}, X_{0}\right]} z_{2}\right)$ and hence $z_{3}$ is independent from $z_{1}$ and $z_{2}$, let $z_{4}$ be a fourth function, such that $\left(z_{1}, z_{2}, z_{3}, z_{4}\right)$ is a system of coordinates. The nonsingular feedback

$$
\begin{aligned}
& v_{1}=L_{X_{0}}^{2} z_{2}+u_{1} L_{X_{1}} L_{X_{0}} z_{2}+u_{2} L_{X_{2}} L_{X_{0}} z_{2} \\
& v_{2}=L_{X_{0}} z_{4}+u_{1} L_{X_{1}} z_{4}+u_{2} L_{X_{2}} z_{4}
\end{aligned}
$$

transforms system (1.5) into

$$
\begin{aligned}
& \dot{z}_{1}=a\left(z_{1}, z_{2}, z_{3}, z_{4}\right) \\
& \dot{z}_{2}=z_{3} \\
& \dot{z}_{3}=v_{1} \\
& \dot{z}_{4}=v_{2}
\end{aligned}
$$


with $a$ a certain smooth function. Since $\Delta_{3}$ spans a distribution of rank 3 and:

$$
\Delta_{3}=\operatorname{Span}\left\{\frac{\partial}{\partial z_{3}}, \frac{\partial}{\partial z_{4}}, \frac{\partial}{\partial z_{2}}+\frac{\partial a}{\partial z_{3}} \frac{\partial}{\partial z_{1}}, \frac{\partial a}{\partial z_{4}} \frac{\partial}{\partial z_{1}}\right\}
$$

the function a cannot depend on $z_{4}$, and then

$$
\Delta_{3}+\left[\Delta_{3}, \Delta_{3}\right]=\operatorname{Span}\left\{\frac{\partial}{\partial z_{3}}, \frac{\partial}{\partial z_{4}}, \frac{\partial}{\partial z_{2}}+\frac{\partial a}{\partial z_{3}} \frac{\partial}{\partial z_{1}}, \frac{\partial^{2} a}{\partial z_{3}^{2}} \frac{\partial}{\partial z_{1}}\right\}
$$

so that the assumption on $\Delta_{3}$ is equivalent to $\frac{\partial^{2} a}{\partial z_{3}^{2}}$ being identically zero on no neighborhood of $\bar{x}$. This proves that system (1.5) has the form (3.4) with the condition (3.5), after the change of coordinates and the nonsingular feedback transformation we just introduced. There remains to prove that system (3.4) cannot be linearizable by endogenous feedback under condition (3.5). This is a consequence of the following lemma 7.1 because if system (3.4) was linearizable by endogenous feedback on a neighborhood of a point $\bar{x}$, then there would exist a pair of linearizing outputs on a neighborhood of this point, and hence the system would also be linearizable by endogenous feedback around any point of that neighborhood, including these, given by condition (3.5), where $\frac{\partial^{2} a}{\partial z_{3}^{2}}$ is non zero.

Lemma 7.1. System (3.4) is not linearizable by endogenous dynamic feedback in any neighborhood of a point $\overline{\bar{z}}=\left(\overline{\bar{z}}_{1}, \overline{\bar{z}}_{2}, \overline{\bar{z}}_{3}, \overline{\bar{z}}_{4}\right)$ such that

$$
\frac{\partial^{2} a}{\partial z_{3}^{2}}\left(\overline{\bar{z}}_{1}, \overline{\bar{z}}_{2}, \overline{\bar{z}}_{3}\right) \neq 0
$$

Proof. Suppose that there exists two linearizing outputs $h_{1}$ and $h_{2}$, smooth functions of a finite number of variables among $z_{1}, z_{2}, z_{3}, z_{4}, v_{1}, v_{2}, \dot{v}_{1}, \dot{v}_{2}$, $\ddot{v}_{1}, \ddot{v}_{2}, \ldots, v_{1}^{(L)}, v_{2}^{(L)}$, with $L$ a non negative integer, defined on an open subset $O \subset \mathbb{R}^{2 L+6}$ containing a point $\left(\overline{\bar{z}}_{1}, \overline{\bar{z}}_{2}, \overline{\bar{z}}_{3}, \overline{\bar{z}}_{4}, \overline{\bar{v}}_{1}, \overline{\bar{v}}_{2}, \ldots, \overline{\bar{v}}_{1}^{(L)}, \overline{\bar{v}}_{2}^{(L)}\right)$ for some $\left(\overline{\bar{v}}_{1}, \overline{\bar{v}}_{2}, \ldots, \overline{\bar{v}}_{1}^{(L)}, \overline{\bar{v}}_{2}^{(L)}\right)$. All variables may be recovered from $h_{1}, h_{2}$ and all their time derivatives so that in particular there exists smooth functions $\psi_{1}$ and $\psi_{2}$ such that

$$
\begin{aligned}
& z_{1}=\psi_{1}\left(h_{1}, \dot{h}_{1}, \ldots, h_{1}^{\left(K_{1,1}\right)}, h_{2}, \dot{h}_{2}, \ldots, h_{2}^{\left(K_{1,2}\right)}\right) \\
& z_{2}=\psi_{2}\left(h_{1}, \dot{h}_{1}, \ldots, h_{1}^{\left(K_{2,1}\right)}, h_{2}, \dot{h}_{2}, \ldots, h_{2}^{\left(K_{2,2}\right)}\right) .
\end{aligned}
$$

This holds in the open set $O$, which may be restricted so that $\frac{\partial^{2} a}{\partial z_{3}^{2}}\left(z_{1}, z_{2}, z_{3}\right)$ does not vanish on $O$. The integer $K_{i, j}$ is the one such that $\psi_{i}$ does not depend on $h_{j}^{\left(K_{i, j}+1\right)}$, but does depend on $h_{j}^{\left(K_{i, j}\right)}$ on $O$, i.e. $\frac{\partial \psi_{i}}{\partial h_{j}^{\left(K_{i, j}\right)}}$ is not identically zero. Then, since (3.4) implies $\dot{z}_{1}=a\left(z_{1}, z_{2}, \dot{z}_{2}\right)$, one has, by substitution, 


$$
\begin{aligned}
\frac{\partial \psi_{1}}{\partial h_{1}} \dot{h}_{1}+\cdots & +\frac{\partial \psi_{1}}{\partial h_{1}^{\left(K_{1,1}\right)}} h_{1}^{\left(K_{1,1}+1\right)}+\frac{\partial \psi_{1}}{\partial h_{2}} \dot{h}_{2}+\cdots+\frac{\partial \psi_{1}}{\partial h_{2}^{\left(K_{1,2}\right)}} h_{2}^{\left(K_{1,2}+1\right)} \\
= & a\left(\psi_{1}, \psi_{2}, \frac{\partial \psi_{2}}{\partial h_{1}} \dot{h}_{1}+\cdots+\frac{\partial \psi_{2}}{\partial h_{1}^{\left(K_{2,1}\right)}} h_{1}^{\left(K_{2,1}+1\right)}\right. \\
& \left.+\frac{\partial \psi_{2}}{\partial h_{2}} \dot{h}_{2}+\cdots+\frac{\partial \psi_{2}}{\partial h_{2}^{\left(K_{2,2}\right)}} h_{2}^{\left(K_{2,2}+1\right)}\right)
\end{aligned}
$$

One must have

$$
K_{1,1}=K_{2,1}, \quad K_{1,2}=K_{2,2}
$$

because the left-hand side in (7.5) depends only on $h_{1}, \dot{h}_{1}, \ldots, h_{1}^{\left(K_{1,1}+1\right)}, h_{2}$, $\dot{h}_{2}, \ldots, h_{2}^{\left(K_{1,2}+1\right)}$ and does depend on $h_{1}^{\left(K_{1,1}+1\right)}$ and $h_{2}^{\left(K_{2,1}+1\right)}$, and the righthand side depends only on $h_{1}, h_{1}, \ldots, h_{1}^{\left(K_{2,1}+1\right)}, h_{2}, h_{2}, \ldots, h_{2}^{\left(K_{2,2}+1\right)}$ and does depend on $h_{1}^{\left(K_{2,1}+1\right)}$ and $h_{2}^{\left(K_{2,2}+1\right)}$ because, since $\frac{\partial^{2} a}{\partial z_{3}^{2}}$ does not vanish on $O, \frac{\partial a}{\partial z_{3}}$ is not identically zero on any open subset of $O$.

Differentiating two times both sides of (7.5) with respect to $h_{j}^{\left(K_{1, j}+1\right)}$, and keeping in mind that, from (7.6), $K_{1, j}=K_{2, j}$, one has (note that neither $\psi_{1}$ nor $\psi_{2}$ nor the partial derivatives of them depend on $\left.h_{j}^{\left(K_{1, j}+1\right)}\right)$ :

$$
\begin{aligned}
0= & \left(\frac{\partial \psi_{2}}{\partial h_{j}^{\left(K_{1, j}\right)}}\right)^{2} \frac{\partial^{2} a}{\partial z_{3}^{2}}\left(\psi_{1}, \psi_{2}, \frac{\partial \psi_{2}}{\partial h_{1}} \dot{h}_{1}+\cdots+\frac{\partial \psi_{2}}{\partial h_{1}^{\left(K_{1,1}\right)}} h_{1}^{\left(K_{1,1}+1\right)}\right. \\
& \left.+\frac{\partial \psi_{2}}{\partial h_{2}} \dot{h}_{2}+\cdots+\frac{\partial \psi_{2}}{\partial h_{2}^{\left(K_{1,2}\right)}} h_{2}^{\left(K_{1,2}+1\right)}\right)
\end{aligned}
$$

for $j \in\{1,2\}$, and hence $\frac{\partial \psi_{2}}{\partial h_{j}^{\left(K_{1, j}\right)}}$ is identically zero on $O$ which contradicts the fact that it was precisely chosen (small enough) not to be identically zero on $O$.

CASE 2.в $\left(m_{0}=m_{1}=2, \delta_{3}=3\right)$. Since $\Delta_{3}$ is integrable of rank 3 , and $\left\{X_{1}, X_{2}\right\}$ is integrable of rank 2 , and contained in $\Delta_{3}$, there are two independent functions $z_{1}$ and $z_{2}$ such that $z_{1}$ and $z_{2}$ are constant along $X_{1}$ and $X_{2}$ and $z_{1}$ constant along the vector fields of $\Delta_{3}$. Let $z_{3}$ be given by $z_{3}=L_{X_{0}} z_{2}$ and $z_{4}$ be such that $\left(z_{1}, z_{2}, z_{3}, z_{4}\right)$ is a system of coordinates. The nonsingular feedback (7.1) transforms system (1.5) into a system of the form (7.2) above, where $a$ depends on $z_{1}$ only because, since $L_{X_{1}} z_{1}=L_{X_{2}} z_{1}=0$, one has $a=\dot{z}_{1}=L_{X_{0}} z_{1}$, and $L_{X_{0}} z_{1}$ is constant along $\Delta_{3}$ because $z_{1}$ is and $\left[X_{0}, \Delta_{3}\right] \subset \Delta_{3} \cdot \dot{z}_{1}=a\left(z_{1}\right)$ clearly implies non-accessibility.

CASE 2.C $\left(m_{0}=m_{1}=2, \delta_{3}=3\right)$. Static feedback linearizability follows from classical results, see $[15,12]$. Let us however describe the coordinates in which the system has the form (2.3.a). Since $\Delta_{3}$ is integrable of rank 3 , there is a function $z_{1}$ such that $\mathrm{d} z_{1}$ is the annihilator of $\Delta_{3}$. Let $z_{2}$ and $z_{3}$ be given by $z_{2}=L_{X_{0}} z_{1}$ and $z_{3}=L_{X_{0}}^{2} z_{1}$, the rank of $\left\{\mathrm{d} z_{1}, \mathrm{~d} z_{2}, \mathrm{~d} z_{3}\right\}$ is 3 
because $\delta_{3}=3$. Let $z_{4}$ be any function such that $\left\{z_{1}, z_{2}, z_{3}, z_{4}\right\}$ is a system of coordinates. The nonsingular feedback

$$
\begin{aligned}
& v_{1}=L_{X_{0} z_{1}}^{3}+u_{1} L_{X_{1}} L_{X_{0}}^{2} z_{1}+u_{2} L_{X_{2}} L_{X_{0}}^{2} z_{1} \\
& v_{2}=L_{X_{0} z_{4}}+u_{1} L_{X_{1}} z_{4}+u_{2} L_{X_{2}} z_{4}
\end{aligned}
$$

transforms system (1.5) into (2.3.a).

CASE $3\left(m_{0}=m_{1}=2, \delta_{3}=4\right)$. As in case 2.c, static feedback linearization follows from classical results, see [15, 12], but we however describe the coordinates in which the system has the form (2.3.b). Because $m_{0}=2, X_{1}$ and $X_{2}$ span an integrable distribution of rank 2 , let $z_{1}$ and $z_{3}$ be two independent functions that annihilate $X_{1}$ and $X_{2}$, and let $z_{2}$ and $z_{4}$ be defined by $z_{2}=L_{X_{0}} z_{1}$ and $z_{4}=L_{X_{0}} z_{3} \cdot \delta_{3}=4$ implies that $\left(z_{1}, z_{2}, z_{3}, z_{4}\right)$ is a system of coordinates, and the following nonsingular feedback

$$
\begin{aligned}
& v_{1}=L_{X_{0}}^{2} z_{1}+u_{1} L_{X_{1}} L_{X_{0}} z_{1}+u_{2} L_{X_{2}} L_{X_{0}} z_{1} \\
& v_{2}=L_{X_{0}}^{2} z_{3}+u_{1} L_{X_{1}} L_{X_{0}} z_{3}+u_{2} L_{X_{2}} L_{X_{0}} z_{3}
\end{aligned}
$$

transforms system (1.5) into (2.3.b).

Cases 4 And $5\left(m_{0}=m_{1}=3, \delta_{3}=3\right.$ oR 4$)$. Since $m_{0}=m_{1}=3$, $\mathcal{M}_{0}=\mathcal{M}_{1}$ spans an integrable distribution of rank 3 . Let $z_{1}$ be a first integral of this distribution. In case $5\left(\delta_{3}=4\right)$, define $z_{2}$ by

$$
z_{2}=L_{X_{0}} z_{1} \text {. }
$$

One then has, for $i \in\{1,2\}, L_{X_{i}} z_{2}=-L_{\left[X_{0}, X_{i}\right]} z_{1}$ because $L_{X_{i}} z_{1} \equiv 0$, $i=1,2$, and hence $\delta_{3}=4$ prevents $L_{X_{1}} z_{2}$ and $L_{X_{2}} z_{2}$ from both vanishing at $\bar{x}$. Up to a permutation of the two controls, we may suppose that

$$
L_{X_{1}} z_{2}(\bar{x}) \neq 0 \text {. }
$$

In case $4\left(\delta_{3}=3\right)$, pick any $z_{2}$ such that (7.9) holds, it is possible since $X_{1}$ does not vanish. Since $L_{X_{1}} z_{1}=0$, the rank of $\left\{\mathrm{d} z_{1}, \mathrm{~d} z_{2}\right\}$ is 2 at point $\bar{x}$. The vector field

$$
\left(L_{X_{2}} z_{2}\right) X_{1}-\left(L_{X_{1}} z_{2}\right) X_{2}
$$

does not vanish at point $\bar{x}, z_{1}$ and $z_{2}$ are two independent functions constant along it, let $z_{3}$ be a third independent first integral of this vector field, and $z_{4}$ be given by

$$
z_{4}=\frac{L_{X_{1}} z_{3}}{L_{X_{1}} z_{2}} .
$$

$\left(z_{1}, z_{2}, z_{3}, z_{4}\right)$ is a system of coordinates because $z_{1}, z_{2}$ and $z_{3}$ are constant along the vector field (7.10) while the Lie derivative of $z_{4}$ along it does not vanish at $\bar{x}$ (a simple computation shows that if it would vanish, the rank of $\mathcal{M}_{0}$ would drop to 2 ). Defining $v_{1}$ and $v_{2}$ according to the nonsingular feedback transformation

$$
\begin{aligned}
& v_{1}=L_{X_{0}} z_{2}+u_{1} L_{X_{1}} L_{X_{0}} z_{1}+u_{2} L_{X_{2}} L_{X_{0}} z_{1} \\
& v_{2}=L_{X_{0}} z_{4}+u_{1} L_{X_{1}} z_{4}+u_{2} L_{X_{2}} z_{4}
\end{aligned}
$$

Esaim: Cocv, June 1997, Vol. 2, Pp. 151-230 
(with a possible permutation of the indices 1 and 2 in the right-hand sides, if needed to get (7.9)) yields, in the above defined coordinates, the normal form (3.7) in case 4 , and (3.9) in case 5 . In both cases, $a_{3}$ is given by

$$
a_{3}=L_{X_{0}} z_{3}-\frac{L_{X_{1}} z_{3}}{L_{X_{1}} z_{2}} L_{X_{0}} z_{2},
$$

$\dot{z}_{3}$ is obtained because

$$
L_{X_{2}} z_{3}=\frac{L_{X_{2}} z_{2}}{L_{X_{1}} z_{2}} L_{X_{1}} z_{3},
$$

and (in case 4) $a_{1}=L_{X_{0}} z_{1}$ depends only on $z_{1}$ because $\delta_{3}=3$ implies that $\Delta_{3}=\mathcal{M}_{0}$ and hence that $L_{X_{0}} z_{1}$ is a first integral of the three dimensional integrable distribution spanned by $\mathcal{M}_{0}$.

In case 4, non-accessibility follows immediately from the normal form (3.7). In case 5, let us prove that system (3.9) is $x$-dynamic linearizable around $(\bar{z}, \bar{v})$ if and only if $\frac{\partial a}{\partial z_{4}}(\bar{z})+\bar{v}_{1} \neq 0$. Let $\left(h_{1}, h_{2}\right)$ be a pair of linearizing outputs, depending on $z$ only.

LEMMA 7.2. Let $h_{1}, h_{2}$ be two functions depending on $z$ only such that $\left(h_{1}, h_{2}\right)$ is a pair of linearizing outputs for system (3.9) on a neighborhood of $(\bar{z}, \bar{v})$. Then the rank of $\left\{\mathrm{d} z_{1}, \mathrm{~d} h_{1}, \mathrm{~d} h_{2}\right\}$ is 2 on a neighborhood of $\bar{z}$.

Proof. If it was not the case, there would be points $\overline{\bar{z}}$, arbitrarily close to $\bar{z}$, where this rank would be 3 , and where $\left(h_{1}, h_{2}\right)$ would still be a pair of linearizing outputs. $z_{1}$ is constant along both control vector fields, and since $\left(h_{1}, h_{2}\right)$ would still be a pair of linearizing outputs, there is, from (2.16)-(2.18), a nonzero linear combination of $X_{1}$ and $X_{2}$, say $Z$, along which both $h_{1}$ and $h_{2}$ are constant. It is impossible that $L_{X_{i}} h_{j}$ vanishes at $\overline{\bar{x}}$ for all $i, j \in\{1,2\}$, so that up to a permutation, we may suppose that $L_{X_{1}} h_{1} \neq 0$. This yields, following the same construction as above construction of coordinates where the system has form (3.9) - a set of coordinates

$$
\left(\zeta_{1}, \zeta_{2}, \zeta_{3}, \zeta_{4}\right)=\left(z_{1}, h_{1}, h_{2}, \frac{L_{X_{1}} h_{2}}{L_{X_{1}} h_{1}}\right)
$$

and a nonsingular feedback $w_{1}=\dot{h}_{1}, w_{2}=\dot{\zeta}_{4}$ such that the system is also of the form (3.9) with $\zeta$ instead of $z$ and $w$ instead of $v$ :

$$
\begin{array}{ll}
\dot{\zeta}_{1}=\zeta_{2} & \dot{\zeta}_{3}=a_{3}\left(\zeta_{1}, \zeta_{2}, \zeta_{3}, \zeta_{4}\right)+\zeta_{4} w_{1} \\
\dot{\zeta}_{2}=w_{1} & \dot{\zeta}_{4}=w_{2}
\end{array}
$$

where $\left(\zeta_{2}, \zeta_{3}\right)$ should be a pair of linearizing outputs. This is impossible from (2.17) because

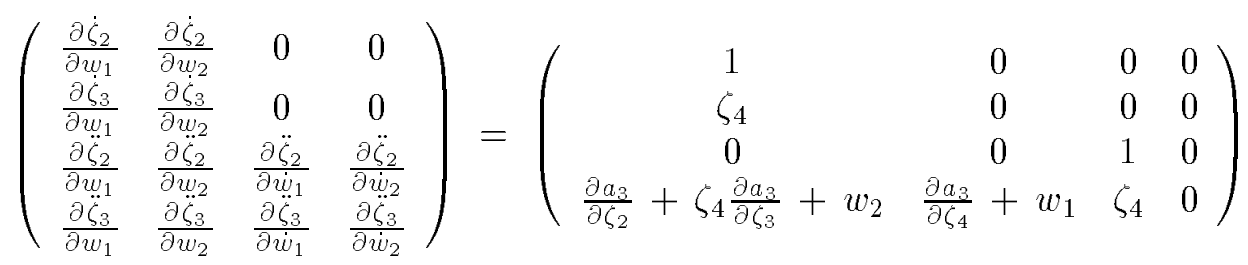

and hence $\frac{\partial a_{3}}{\partial \zeta_{4}}+w_{1}$ should be identically zero on an open set, which is absurd because its derivative with respect to $w_{1}$ is 1 . 
From this lemma $7.2, z_{1}$ is a function of the two linearizing functions, and therefore one may replace $h_{1}$ or $h_{2}$ by $z_{1}$ in $\left(h_{1}, h_{2}\right)$ and still have a pair of linearizing outputs. Let for instance $h_{1}=z_{1}$, then (2.16) is automatically satisfied, and (2.17) implies that $h_{2}$ must depend on $z_{1}, z_{2}, z_{3}$ only because

$$
\left(\begin{array}{cccc}
\frac{\partial \dot{h}_{1}}{\partial v_{1}} & \frac{\partial \dot{h}_{1}}{\partial v_{2}} & 0 & 0 \\
\frac{\partial \dot{h}_{2}}{\partial v_{1}} & \frac{\partial \dot{h}_{2}}{\partial \vartheta_{2}} & 0 & 0 \\
\frac{\partial \ddot{h}_{1}}{\partial \vartheta_{1}} & \frac{\partial \ddot{h}_{1}}{\partial \vartheta_{2}} & \frac{\partial \ddot{h}_{1}}{\partial \dot{\vartheta}_{1}} & \frac{\partial \ddot{h}_{1}}{\partial \dot{\vartheta}_{2}} \\
\frac{\partial \dot{h}_{2}}{\partial v_{1}} & \frac{\partial \ddot{h}_{2}}{\partial v_{2}} & \frac{\partial \dot{h}_{2}}{\partial \dot{v}_{1}} & \frac{\partial \ddot{h}_{2}}{\partial \dot{v}_{2}}
\end{array}\right)=\left(\begin{array}{cccc}
0 & 0 & 0 & 0 \\
* & \frac{\partial h_{2}}{\partial z_{4}} & 0 & 0 \\
1 & 0 & 0 & 0 \\
* & * & * & \frac{\partial h_{2}}{\partial z_{4}}
\end{array}\right),
$$

and the independence condition in proposition 2.14 implies (3.10). Conversely, if $(3.10)$ is satisfied, system (3.9) is $x$-dynamic linearizable with $\left(z_{1}, z_{3}\right)$ as a pair of linearizing outputs, because $z_{2}$ is $\dot{z}_{1}$, and $z_{4}$ is (inverse function theorem) a function of $\dot{z}_{3}, z_{1}, z_{2}, z_{3}, v_{1}$, i.e. of $\dot{z}_{3}, z_{1}, \dot{z}_{1}, z_{3}, \ddot{z}_{1}$.

CASE $6\left(m_{0}=3, m_{1}=4\right)$. Let us first clarify the correspondence between the conditions in terms of differential forms and these in terms of vector fields. Since the form $\omega_{1}$ is defined by (3.15) and involves only the four variables $x, \mathrm{~d} \omega_{1}$ must be of the form (3.19) because there is only four variables. Let us prove that, as written just after (3.19),

$$
\widetilde{X} \in\left\{\omega_{1}, \eta_{1}, \eta_{2}\right\}^{\perp}
$$

From the definitions of $\omega_{1}$ and $\tilde{X}$, one has $\left\langle\omega_{1},[\widetilde{X}, Y]\right\rangle=0$ for $Y=X_{1}$ and for $Y=X_{2}$ and for $Y=\left[X_{1}, X_{2}\right]$, but one also has $\left\langle\omega_{1}, \widetilde{X}\right\rangle=\left\langle\omega_{1}, Y\right\rangle=0$ for these $Y$ 's, and hence, from the classical formula [25, II-(1.10)] linking Lie Bracket and exterior derivative, $\left\langle\omega_{1},[\tilde{X}, Y]\right\rangle=0$ implies $\mathrm{d} \omega_{1}(\widetilde{X}, Y)=$ 0 , but from (3.19), and using again $\left\langle\omega_{1}, \tilde{X}\right\rangle=\left\langle\omega_{1}, Y\right\rangle=0$, this reads: $\left\langle\eta_{1}, \tilde{X}\right\rangle\left\langle\eta_{2}, Y\right\rangle-\left\langle\eta_{1}, Y\right\rangle\left\langle\eta_{2}, \tilde{X}\right\rangle=0$. Since the three vectors $X_{1}, X_{2},\left[X_{1}, X_{2}\right]$ are linearly independent $\left(m_{0}=3\right)$ and the two differential forms $\eta_{1}$ and $\eta_{2}$ are also linearly independent $\left(m_{1}=4\right.$ implies that $\mathrm{d} \omega_{1} \wedge \omega_{1}=\eta_{1} \wedge \eta_{2} \wedge \omega_{1}$ does not vanish), the last equality implies $\left\langle\eta_{1}, \tilde{X}\right\rangle=\left\langle\eta_{2}, \widetilde{X}\right\rangle=0$, and this proves $(7.11)$.

We then have the following

LEMMA 7.3. Condition (3.12) is equivalent to condition (3.16). If (3.12) or (3.16) holds, condition (3.13) is equivalent to condition (3.17) and condition (3.15) is equivalent to condition (3.18).

Proof. From the definition of $\omega_{1},(3.12)$ may be written $\left\langle\omega_{1},\left[X_{0}, \tilde{X}\right]\right\rangle=0$, or also $\left\langle\omega_{1},[F, \tilde{X}]\right\rangle=0$ because $[F, \widetilde{X}]=\left[X_{0}, \tilde{X}\right]+u_{1}\left[X_{1}, \tilde{X}\right]+u_{2}\left[X_{2}, \tilde{X}\right]$ and the last two terms vanish on $\omega_{1}$. From the classical identity $(2.10)$ and the fact that $\left\langle\omega_{1}, \tilde{X}\right\rangle$ is zero, $\left\langle\omega_{1},[F, \tilde{X}]\right\rangle=0$ is equivalent to $\left\langle\dot{\omega}_{1}, \tilde{X}\right\rangle=0$, which is equivalent, from (7.11) to $\dot{\omega}_{1}$ being a linear combination of $\omega_{1}, \eta_{1}$ and $\eta_{2}$. This is (3.16).

Let us proceed to prove that (3.13) is equivalent to (3.17) if (3.16) holds. Consider the three vector fields $\widetilde{X}, \frac{\partial}{\partial u_{1}}, \frac{\partial}{\partial u_{2}}$ in the six variables $x, u$. Their annihilator is $\left\{\omega_{1}, \eta_{1}, \eta_{2}\right\}$. Now consider the six vector fields obtained by adding the Lie brackets of these by $F:\left\{\tilde{X}, \frac{\partial}{\partial u_{1}}, \frac{\partial}{\partial u_{2}},[F, \widetilde{X}],\left[F, \frac{\partial}{\partial u_{1}}\right],\left[F, \frac{\partial}{\partial u_{2}}\right]\right\}$. From the classical identity (2.10), a form $\omega$ annihilates all these at a point Esaim: Cocv, JunE 1997, Vol. 2, PP. 151-230 
if and only if $\omega$ and $\dot{\omega}$ annihilate the original three at this point, i.e. if and only if both $\omega$ and $\dot{\omega}$ are linear combinations of $\omega_{1}, \eta_{1}$ and $\eta_{2}$ at this point. It is the case of $\omega_{1}$ because (3.16) holds, and the rank of these six vector fields therefore cannot be more than 5 ; it is equal to 5 exactly at points where the time-derivative of any linear combination of $\eta_{1}$ and $\eta_{2}$ is linearly independent from $\omega_{1}, \eta_{1}$ and $\eta_{2}$, i.e. at points where (3.17) holds. Now this rank is 5 exactly at points where $(3.13)$ holds because $\left[F, \frac{\partial}{\partial u_{i}}\right]=X_{i}$ and therefore these six vector fields have the same rank as:

$$
\left\{X_{1}, X_{2},[F, \widetilde{X}], \frac{\partial}{\partial u_{1}}, \frac{\partial}{\partial u_{2}}\right\} .
$$

Let us proceed to prove that (3.15) is equivalent to (3.18) if (3.16) holds. Consider the five vector fields $\left\{X_{1}, X_{2},\left[X_{1}, X_{2}\right], \frac{\partial}{\partial u_{1}}, \frac{\partial}{\partial u_{2}}\right\}$ in the six variables $x, u$. Their annihilator is $\left\{\omega_{1}\right\}$. Now consider the ten vector fields obtained by adding the Lie brackets of these by $F$ :

$$
\left\{X_{1}, X_{2},\left[X_{1}, X_{2}\right], \frac{\partial}{\partial u_{1}}, \frac{\partial}{\partial u_{2}},\left[F, X_{1}\right],\left[F, X_{2}\right],\left[F,\left[X_{1}, X_{2}\right]\right],\left[F, \frac{\partial}{\partial u_{1}}\right],\left[F, \frac{\partial}{\partial u_{2}}\right]\right\} .
$$

A form that annihilates all these vector fields at a point must be collinear to $\omega_{1}$ at this point because it has to annihilate at least the five original ones. The form $\omega_{1}$ vanishes on all these vector fields exactly at the point where $\omega_{1}$ and $\dot{\omega}_{1}$ vanish on the five original vector fields, i.e. (since these five are linearly independent) exactly at points where the rank of $\left\{\omega_{1}, \dot{\omega}_{1}\right\}$ drops to 1. Therefore, the rank of the ten vector fields is 6 at points where (3.18) holds, and 5 at points where it does not hold. But these ten vector have the same rank as:

$$
\begin{gathered}
\left\{X_{1}, X_{2},\left[X_{1}, X_{2}\right], \frac{\partial}{\partial u_{1}}, \frac{\partial}{\partial u_{2}},\left[X_{0}, X_{1}\right],\left[X_{0}, X_{2}\right],\right. \\
\left.\left[X_{0}+u_{1} X_{1}+u_{2} X_{2},\left[X_{1}, X_{2}\right]\right]\right\},
\end{gathered}
$$

and this has rank 6 if and only if (3.15) holds.

Let us now prove necessity of the conditions (3.12)-(3.13)-(3.15), or (3.16)(3.17)-(3.18). Lemma 7.3, that we have now proved, allows us to simply prove that (3.12) is necessary first, and then to prove that (3.17) and (3.18) are necessary.

Suppose that there exists a pair of linearizing outputs $\left(h_{1}, h_{2}\right)$ with $h_{1}$ and $h_{2}$ depending on $x$ only. We use conditions (2.16) and (2.17) from proposition 2.14 to derive the necessary condition (3.12). We have

$$
\dot{h}_{i}=L_{X_{0}} h_{i}+u_{1} L_{X_{1}} h_{i}+u_{2} L_{X_{2}} h_{i} .
$$

Equation (2.16) implies that the rank of

$$
\left(\begin{array}{cc}
L_{X_{1}} h_{1} & L_{X_{2}} h_{1} \\
L_{X_{1}} h_{2} & L_{X_{2}} h_{2}
\end{array}\right)
$$

is one. Since $m_{1}=4$, the functions $L_{X_{1}} h_{1}$ and $L_{X_{2}} h_{1}$ cannot vanish together; without loss of generality, suppose that $L_{X_{1}} h_{1}$ does not vanish at the point under consideration. Then, with $\lambda$ the function given by $\lambda=L_{X_{2}} h_{1} / L_{X_{1}} h_{1}$, and defining the vector field $Z_{2}$ by

$$
Z_{2}=X_{2}-\lambda X_{1}
$$


one has

$$
L_{Z_{2}} h_{1}=L_{Z_{2}} h_{2}=0 .
$$

Define the vector fields $Z_{0}$ and $Z_{1}$ by

$$
Z_{0}=X_{0}-\frac{L_{X_{0}} h_{1}}{L_{X_{1}} h_{1}} X_{1}, \quad Z_{1}=\frac{1}{L_{X_{1}} h_{1}} X_{1} .
$$

The systems (1.5) then reads

$$
\dot{x}=Z_{0}+w_{1} Z_{1}+u_{2} Z_{2}
$$

with $w_{1}$ defined as follows $\left(\left(u_{1}, u_{2}\right) \mapsto\left(w_{1}, u_{2}\right)\right.$ defines a regular static feedback:

$$
\begin{aligned}
w_{1}=\dot{h}_{1} & =L_{X_{0}} h_{1}+u_{1} L_{X_{1}} h_{1}+u_{2} L_{X_{2}} h_{1} \\
& =L_{X_{0}} h_{1}+\left(u_{1}+\lambda u_{2}\right) L_{X_{1}} h_{1} .
\end{aligned}
$$

Then $\dot{h}_{1}$ and $\dot{h}_{2}$ may be written:

$$
\begin{aligned}
& \dot{h}_{1}=w_{1} \\
& \dot{h}_{2}=L_{Z_{0}} h_{2}+w_{1} L_{Z_{1}} h_{2} .
\end{aligned}
$$

The second time-derivatives are then given by:

$$
\begin{aligned}
\ddot{h}_{1}= & \dot{w}_{1} \\
\ddot{h}_{2}= & L_{Z_{0}}^{2} h_{2}+w_{1}\left(L_{Z_{1}} L_{Z_{0}}+L_{Z_{0}} L_{Z_{1}}\right) h_{2}+w_{1} L_{Z_{1}}^{2} h_{2}+\dot{w}_{1} L_{Z_{1}} h_{2} \\
& +\left(L_{Z_{2}} L_{Z_{0}} h_{2}+w_{1} L_{Z_{2}} L_{Z_{1}} h_{2}\right) u_{2} .
\end{aligned}
$$

The function $\ddot{h}_{2}$ must not depend on $u_{2}$-this is (2.17)- and hence

$$
L_{Z_{2}} L_{Z_{0}} h_{2}=L_{Z_{2}} L_{Z_{1}} h_{2}=0 .
$$

Now, on one hand, from (7.14)-(7.18)-(7.16), $L_{Z_{1}} h_{1}$ is identically equal to 1 , and $L_{Z_{2}} h_{1}, L_{Z_{0}} h_{1}$ and $L_{Z_{2}} h_{1}$ are identically zero, so that $L_{\left[Z_{2}, Z_{1}\right]} h_{1}$ and $L_{\left[Z_{2}, Z_{0}\right]} h_{1}$ are obviously zero, and on the other hand, since $L_{Z_{2}} h_{2}$ is identically zero from (7.14), $L_{Z_{2}} L_{Z_{1}} h_{2}$ is equal to $L_{\left[Z_{2}, Z_{1}\right]} h_{2}$ and $L_{Z_{2}} L_{Z_{0}} h_{2}$ is equal to $L_{\left[Z_{2}, Z_{0}\right]} h_{2}$; this and $(7.20)$ above implies:

$$
\begin{aligned}
& L_{\left[Z_{2}, Z_{1}\right]} h_{1}=L_{\left[Z_{2}, Z_{1}\right]} h_{2}=0, \\
& L_{\left[Z_{2}, Z_{0}\right]} h_{1}=L_{\left[Z_{2}, Z_{0}\right]} h_{2}=0 .
\end{aligned}
$$

The two independent functions $h_{1}$ and $h_{2}$ are, from (7.21) and (7.14), constant along the vector fields $Z_{2}$ and $\left[Z_{1}, Z_{2}\right]$, which are linearly independent because $m_{1}=3$. This implies that the distribution spanned by these two vector fields is integrable, and therefore that the Lie Bracket $\left[Z_{2},\left[Z_{1}, Z_{2}\right]\right]$ is a linear combination of $Z_{2}$ and $\left[Z_{1}, Z_{2}\right]$. From (7.13) and (7.15), the Lie bracket $\left[Z_{2},\left[Z_{1}, Z_{2}\right]\right]$ is equal to $\lambda\left[Z_{2},\left[X_{1}, X_{2}\right]\right]+\left(L_{X_{1}} \lambda\right)\left[X_{1}, X_{2}\right]+$ $\left(\left(L_{X_{1}} \lambda\right)^{2}-L_{X_{2}} L_{X_{1}} \lambda\right) X_{1}$. Hence, $\left[Z_{2},\left[X_{1}, X_{2}\right]\right]$ must be a linear combination of $X_{1}, X_{2}$ and $\left[X_{1}, X_{2}\right]$. This implies, from the definition of the characteristic vector field $\widetilde{X}$-see $(3.11)$ - that $Z_{2}$ is collinear to $\widetilde{X}$ :

$$
Z_{2}=\alpha \tilde{X}
$$

with $\alpha$ a nonzero function. Since the vector fields annihilating $\mathrm{d} h_{1}$ and $\mathrm{d} h_{2}$ are the linear combinations of $Z_{2}$ and $\left[Z_{2}, Z_{1}\right],(7.21)$ and (7.22) imply that $\left[Z_{2}, Z_{0}\right]$ is a linear combination of $Z_{2}$ and $\left[Z_{2}, Z_{1}\right]$, which implies in particular Esaim: COCV, JUNe 1997, VOL. 2, PP. 151-230 
that it is a linear combination of $X_{1}, X_{2}$ and $\left[X_{1}, X_{2}\right]$. From (7.23), this implies condition (3.12). We have proved the necessity of condition (3.12).

Let us now prove that existence of $h_{1}$ and $h_{2}$ with the above properties imply (3.17)-(3.18). From above, $\mathrm{d} h_{1}$ and $\mathrm{d} h_{2}$ vanish on $\widetilde{X}$ and $\left[Z_{2}, \widetilde{X}\right]$, and are linearly independent because $\left(h_{1}, h_{2}\right)$ is a pair of linearizing outputs; hence, from the definition of $\omega_{1}$ and the fact that both $\tilde{X}$ and $\left[Z_{2}, \tilde{X}\right]$ are linear combinations of $X_{1}, X_{2}$ and $\left[X_{1}, X_{2}\right]$, the form $\omega_{1}$ is a linear combination of $\mathrm{d} h_{1}$ and $\mathrm{d} h_{2}$, i.e. there exists some functions $\lambda_{1}$ and $\lambda_{2}$ such that

$$
\omega_{1}=\lambda_{1} \mathrm{~d} h_{1}+\lambda_{2} \mathrm{~d} h_{2} .
$$

Computing the time-derivative of this yields

$$
\dot{\omega}_{1}=\lambda_{1} \mathrm{~d} \dot{h}_{1}+\lambda_{2} \mathrm{~d} \dot{h}_{2}+\dot{\lambda}_{1} \mathrm{~d} h_{1}+\dot{\lambda}_{2} \mathrm{~d} h_{2} .
$$

This implies (3.18) because on one hand the two functions $\lambda_{1}$ and $\lambda_{2}$ do not vanish simultaneously because $\omega_{1}$ does not vanish, and on the other hand $\mathrm{d} h_{1}, \mathrm{~d} h_{2}, \mathrm{~d} \dot{h}_{1}$ and $\mathrm{d} \dot{h}_{2}$ are linearly independent because $\left(h_{1}, h_{2}\right)$ is a pair of linearizing outputs. Condition (3.16), already proved because it is equivalent to (3.12) from lemma 7.3, implies:

$$
\dot{\omega}_{1}=\mu_{0} \omega_{1}+\mu_{1} \eta_{1}+\mu_{2} \eta_{2}
$$

for some functions $\mu_{0}, \mu_{1}, \mu_{2}$. (3.18) implies that $\mu_{1}$ and $\mu_{2}$ do not vanish simultaneously. Let $\eta$ be $\eta_{1}$ if $\mu_{2}$ does not vanish, and $\eta_{2}$ if $\mu_{2}$ vanishes. Then $\left\{\omega_{1}, \dot{\omega}_{1}, \eta\right\}$ is another basis for the annihilator of $\tilde{X}$. Since $\mathrm{d} h_{1}$ and $\mathrm{d} h_{2}$ are in the annihilator of $\left\{\tilde{X},\left[Z_{2}, \tilde{X}\right]\right\}$, they are linear combinations of $\omega_{1}, \dot{\omega}_{1}$ and $\eta$. Since $\left\{\mathrm{d} h_{1}, \mathrm{~d} h_{2}\right\}$ is a linearizing Pfaffian system, this implies, from Proposition 2.8, that $\left\{\omega_{1}, \eta\right\}$ is a linearizing Pfaffian system, and hence that $\omega_{1}, \eta$ and all their time derivatives are linearly independent, and in particular $\omega_{1}, \dot{\omega}_{1}, \ddot{\omega}_{1}, \eta, \dot{\eta}$ has rank 5 , but from the above construction, it is also the rank of $\omega_{1}, \eta_{1}, \dot{\eta}_{1}, \eta_{2}, \dot{\eta}_{2}$. This proves (3.17).

According to the remarks just after the proof of lemma 7.3 , we have now proved the necessity of either (3.12)-(3.13)-(3.15), or (3.16)-(3.17)-(3.18). Let us prove sufficiency, and at the same time validity of the way of building linearizing outputs given in the theorem. Again, from lemma 7.3, it is enough to prove sufficiency of (3.16)-(3.17)-(3.18).

From (3.16) and (3.18), equation (3.19) implies

$$
\mathrm{d} \omega_{1}=\omega_{1} \wedge \Gamma^{\prime}+k \dot{\omega}_{1} \wedge \eta
$$

where $k$ is a non-vanishing function and $\eta$ is either $\eta_{1}$ or $\eta_{2}$, and then (3.17) implies

$$
\operatorname{rank}\left\{\omega_{1}, \dot{\omega}_{1}, \ddot{\omega}_{1}, \eta, \dot{\eta}\right\}=5
$$

Let $\alpha_{1}$ and $\alpha_{2}$ be some (non vanishing simultaneously) functions such that $\left\{\omega_{1}, \alpha_{1} \dot{\omega}_{1}+\alpha_{2} \eta\right\}$ is a basis of the annihilator of $\left\{X_{1}, X_{2}\right\}$. Then $\left\{\omega_{1}, \dot{\omega}_{1}, \eta\right.$, $\left.\alpha_{1} \ddot{\omega}_{1}+\alpha_{2} \dot{\eta}\right\}$ is a basis of $\operatorname{Span}\{\mathbf{d} \mathbf{x}\}$-all four are in $\operatorname{Span}\{\mathbf{d} \mathbf{x}\}$ because $\alpha_{1} \dot{\omega}_{1}+\alpha_{2} \eta$ vanishes on $X_{1}$ and $X_{2}$, and they are independent from $(7.27)$ and $\left\{\omega_{1}, \dot{\omega}_{1}, \ddot{\omega}_{1}, \eta, \dot{\eta}, \alpha_{1} \omega_{1}^{(3)}+\alpha_{2} \ddot{\eta}\right\}$ is a basis of $\operatorname{Span}\{\mathrm{dx}, \mathrm{d} \mathbf{u}\}$ because of 
(7.27) and the fact that $X_{1}$ and $X_{2}$ are supposed to have rank 2 , and then an easy induction shows that

$$
\left\{\omega_{1}, \dot{\omega}_{1}, \ddot{\omega}_{1}, \ldots, \omega_{1}^{(j+2)}, \eta, \dot{\eta}, \ddot{\eta}, \ldots, \eta^{(j+1)}, \alpha_{1} \omega_{1}^{(j+3)}+\alpha_{2} \eta^{(j+2)}\right\}
$$

is a basis of $\operatorname{Span}\left\{\mathrm{d} x, \mathrm{~d} u, \mathrm{~d} \dot{u}, \ldots, \mathrm{d} u^{(j)}\right\}$ for all $j \geq 0$. This implies that $\left\{\omega_{1}, \eta\right\}$ is a linearizing Pfaffian system (see definition 2.5).

Let us now build a pair of linearizing outputs as explained in the theorem. If $h_{1}$ is built as indicated, i.e. such that

$$
L_{\tilde{X}} h_{1}=0 \text { and } \operatorname{rank}\left\{\omega_{1}, \dot{\omega}_{1}, \mathrm{~d} h_{1}\right\}=3,
$$

the Pfaffian system $\left\{\mathrm{d} h_{1}, \omega_{1}\right\}$ is integrable because (7.26) and the fact that $\mathrm{d} h_{1}$ is a linear combination of $\omega_{1}, \dot{\omega}_{1}, \eta \mathrm{imply} \mathrm{d} \omega_{1} \wedge \omega_{1} \wedge \mathrm{d} h_{1}=0$. Let $h_{2}$ be a second function such that $\left\{\mathrm{d} h_{1}, \mathrm{~d} h_{2}\right\}$ is another basis for $\left\{\omega_{1}, \mathrm{~d} h_{1}\right\}$. These $\mathrm{d} h_{1}$ and $\mathrm{d} h_{2}$ are obviously linear combinations of $\omega_{1}, \dot{\omega}_{1}$ and $\eta$, but this may be inverted: $\omega_{1}$ is a linear combination of $\mathrm{d} h_{1}$ and $\mathrm{d} h_{2}$, and $\eta$ is, from (7.28) a linear combination of $\omega_{1}, \dot{\omega}_{1}, \mathrm{~d} h_{1}$, and hence of $\mathrm{d} h_{1}, \mathrm{~d} h_{2}, \mathrm{~d} \dot{h}_{1}$ and $\mathrm{d} \dot{h}_{2}$. Since $\left\{\omega_{1}, \eta\right\}$ is a linearizing Pfaffian system, $\left(\mathrm{d} h_{1}, \mathrm{~d} h_{2}\right)$ is, from Proposition 2.8, also a linearizing Pfaffian system, and $\left(h_{1}, h_{2}\right)$ is a pair of linearizing outputs from Proposition 2.6. This completes the proof of sufficiency.

Let us now prove the assertions concerned with the "normal form". The normal form itself is a consequence of the following lemma:

Lemma 7.4 ("EngeL's nORMAL FORM"). Let $X_{1}$ and $X_{2}$ be two vector fields in $\mathbb{R}^{4}$ and let $\bar{x} \in \mathbb{R}^{4}$ be such that

$\operatorname{rank}_{\mathbb{R}}\left\{X_{1}(\bar{x}), X_{2}(\bar{x})\right\}=2$, $\operatorname{rank}_{\mathbb{R}}\left\{X_{1}(\bar{x}), X_{2}(\bar{x}),\left[X_{1}, X_{2}\right](\bar{x})\right\}=3$, $\operatorname{rank}_{\mathbb{R}}\left\{X_{1}(\bar{x}), X_{2}(\bar{x}),\left[X_{1}, X_{2}\right](\bar{x}),\left[X_{1},\left[X_{1}, X_{2}\right]\right](\bar{x}),\left[X_{2},\left[X_{1}, X_{2}\right]\right](\bar{x})\right\}=4$.

Then there exists four functions $\alpha_{11}, \alpha_{12}, \alpha_{21}, \alpha_{22}$ and a set of coordinates $\left(z_{1}, z_{2}, z_{3}, z_{4}\right)$ such that the matrix $\left(\begin{array}{ll}\alpha_{11}(\bar{x}) & \alpha_{12}(\bar{x}) \\ \alpha_{21}(\bar{x}) & \alpha_{22}(\bar{x})\end{array}\right)$ is invertible, and, locally around $\bar{x}$,

$$
\begin{aligned}
\alpha_{11} X_{1}+\alpha_{21} X_{2} & =\frac{\partial}{\partial z_{1}}+z_{3} \frac{\partial}{\partial z_{2}}+z_{4} \frac{\partial}{\partial z_{3}} \\
\alpha_{12} X_{1}+\alpha_{22} X_{2} & =\frac{\partial}{\partial z_{4}} .
\end{aligned}
$$

The proof is very classical, see for example [6]. Now, by assumption, the vector fields $X_{1}$ and $X_{2}$ satisfy these assumptions, and the feedback

$$
\left(\begin{array}{l}
u_{1} \\
u_{2}
\end{array}\right)=\left(\begin{array}{ll}
\alpha_{11} & \alpha_{12} \\
\alpha_{21} & \alpha_{22}
\end{array}\right)\left(\begin{array}{l}
v_{1} \\
v_{2}
\end{array}\right)-\left(\begin{array}{c}
L_{X_{0}} z_{1} \\
L_{X_{0}} z_{4}
\end{array}\right)
$$

yields the equations (3.20) in the coordinates given by lemma 7.4. The fact that the coordinate-free and feedback invariant conditions (3.16), (3.17), (3.18) translate into $(3.21),(3.22),(3.23)$ respectively is a routine computation from

$$
\begin{array}{lll}
\omega_{1}=\mathrm{d} z_{2}-z_{3} \mathrm{~d} z_{1} & \dot{\omega}_{1}=\mathrm{d} f_{2}+v_{1} \mathrm{~d} z_{3}-\left(f_{3}+z_{4} v_{1}\right) \mathrm{d} z_{1} \\
\eta_{1}=\mathrm{d} z_{1}, & \dot{\eta}_{1}=\mathrm{d} v_{1} \\
\eta_{2}=\mathrm{d} z_{3}, & \dot{\eta}_{2}=\mathrm{d} f_{3}+v_{1} \mathrm{~d} z_{4} v_{1}+z_{4} \mathrm{~d} v_{1} .
\end{array}
$$


Alternative proof of Case 6. Here we suppose in addition that we are at Brunovský-regular point, i.e. the rank condition (4.5) holds, and we give a proof for case 6 based on the infinitesimal Brunovský form. To give a thorough treatment of case 6 , one should consider the case when the rank in (4.5) is three in a neighborhood - then there is a different infinitesimal Brunovsky form, as in the second point of proposition 2.11, and also points where it three, while being 4 in an open dense set of a neighborhood - at such points, an infinitesimal Brunovský form does not exist but one might conclude by density.

Condition (4.5) implies, see proposition 2.11, that if two forms $\omega_{1}$ and $\omega_{2}$ make up a basis of $\hat{\mathcal{D}}_{2}^{\perp}$, then $\left\{\omega_{1}, \omega_{2}, \dot{\omega}_{1}, \dot{\omega}_{2}\right\}$ is a basis of $\operatorname{Span}\{\mathrm{d} \mathbf{x}\}$. In addition, $\omega_{1}$ may be taken in $\mathcal{M}_{0}^{\perp}$ (i.e. $\left\{\omega_{1}\right\}$ is the first derived system of the Pfaffian system $\left.\left\{\omega_{1}, \omega_{2}\right\}\right)$. Then we have:

$$
\begin{array}{ll}
\mathrm{d} \omega_{1} \equiv \omega_{2} \wedge\left(\delta_{1} \dot{\omega}_{1}+\delta_{2} \dot{\omega}_{2}\right) & \text { modulo } \omega_{1} \\
\mathrm{~d} \omega_{2} \equiv \gamma \dot{\omega}_{1} \wedge \dot{\omega}_{2} & \text { modulo }\left\{\omega_{1}, \omega_{2}\right\} .
\end{array}
$$

Since on one hand the rank of $\mathcal{M}_{1}$ is constant equal to 4 , and on the other hand the rank of $\mathcal{M}_{0}$ is constant equal to 3 ,

$$
\left\{\begin{aligned}
\delta_{1} \text { and } \delta_{2} & \text { do not vanish simultaneously, } \\
\gamma & \text { does not vanish. }
\end{aligned}\right.
$$

A computations shows that:

$$
\operatorname{Span}\{\tilde{X}\}=\left\{\omega_{1}, \omega_{2}, \delta_{1} \dot{\omega}_{1}+\delta_{2} \dot{\omega}_{2}\right\}^{\perp} .
$$

The proof of characterization (3.12) relies on the following lemma, proved further: LEMма 7.5. The following three properties are equivalent:

(i) There exist two invertible matrices $J_{1}$ and $J_{2}$ of degree zero and three functions $a, h_{1}$ and $h_{2}$, all defined on a neighborhood of the point $\mathcal{X}$, such that

$$
\left(\begin{array}{l}
\mathrm{d} h_{1} \\
\mathrm{~d} h_{2}
\end{array}\right)=J_{1}\left(\begin{array}{cc}
1 & -a \frac{\mathrm{d}}{\mathrm{dt}} \\
0 & 1
\end{array}\right) J_{2}\left(\begin{array}{l}
\omega_{1} \\
\omega_{2}
\end{array}\right) .
$$

(ii) $\delta_{2}=0$ on a neighborhood of $\mathcal{X}$.

(iii) (3.12) holds on a neighborhood of $\mathcal{X}$.

This is enough to conclude. Indeed, sufficiency in case 6 of theorem 3.1 is obvious because, from proposition 2.12, point (i) implies $x$-dynamic linearizability. Let us prove necessity: if system (1.5) is $x$-dynamic linearizable in a neighborhood of a point $\overline{\mathcal{X}}$, then from propositions 2.12 and 2.13 , there is an open set $U_{0}$, dense in a neighborhood of $\overline{\mathcal{X}}$, such that point (i) holds for all $\mathcal{X} \in U_{0}$. From the lemma, this implies that $\delta_{2}$ is zero on $U_{0}$. Hence it is zero on a neighborhood of $\overline{\mathcal{X}}$. This completes the proof of case 6 of theorem 3.1, the normal form being proved the same way as in the first proof.

Proof. (ii) $\Leftrightarrow$ (iii): We have, from (7.33) and identity (2.10),

$$
\begin{aligned}
0=\frac{\mathrm{d}}{\mathrm{dt}}\left\langle\delta_{1} \omega_{1}+\delta_{2} \omega_{2}, \tilde{X}\right\rangle= & \left\langle\dot{\delta}_{1} \omega_{1}+\dot{\delta}_{2} \omega_{2}+\delta_{1} \dot{\omega}_{1}+\delta_{2} \dot{\omega}_{2}, \tilde{X}\right\rangle \\
& +\left\langle\delta_{1} \omega_{1}+\delta_{2} \omega_{2},\left[X_{0}+u_{1} X_{1}+u_{2} X_{2}, \tilde{X}\right]\right\rangle,
\end{aligned}
$$

which, from the fact that $\left\langle\omega_{i}, \tilde{X}\right\rangle$ and $\left\langle\omega_{1},\left[X_{i}, \tilde{X}\right]\right\rangle$ are identically zero for $i=1,2$, yields

$$
\delta_{1}\left\langle\omega_{1},\left[X_{0}, \tilde{X}\right]\right\rangle+\delta_{2}\left\langle\omega_{2},\left[X_{0}+u_{1} X_{1}+u_{2} X_{2}, \tilde{X}\right]\right\rangle=0
$$

which implies, since $\delta_{1}$ and $\delta_{2}$ do not vanish simultaneously and $\left[X_{0}+u_{1} X_{1}+\right.$ $\left.u_{2} X_{2}, \tilde{X}\right]$ does not vanish, that $\delta_{2}=0$ is equivalent to $\left\langle\omega_{1},\left[X_{0}, \tilde{X}\right]\right\rangle=0$, i.e. to (3.12). 
(ii) $\Rightarrow\left(\right.$ i) : Since $\delta_{2}=0,(7.31)$ implies that $\left\{\omega_{1}, \omega_{2}, \dot{\omega}_{1}\right\}$ is the characteristic system of $\omega_{1}$ and therefore is integrable. In particular, there exists a function $h_{2}$ such that

$$
\mathrm{d} h_{2}=\lambda_{0} \omega_{1}+\lambda_{1} \dot{\omega}_{1}+\lambda_{2} \omega_{2}
$$

with a non-vanishing $\lambda_{2}$; then

$$
\mathrm{d} \omega_{1} \equiv \widetilde{\delta}_{1} \mathrm{~d} h_{2} \wedge \dot{\omega} \text { modulo } \omega_{1}
$$

which implies that $\left\{\omega_{1}, \mathrm{~d} h_{2}\right\}$ is integrable and in particular that there exists a function $h_{1}$ such that

$$
\left(\begin{array}{c}
\mathrm{d} h_{1} \\
\mathrm{~d} h_{2}
\end{array}\right)=\left(\begin{array}{cc}
\mu_{1} & \mu_{2} \\
0 & 1
\end{array}\right)\left(\begin{array}{c}
\omega_{1} \\
\mathrm{~d} h_{2}
\end{array}\right)=\left(\begin{array}{cc}
\mu_{1} & \mu_{2} \\
0 & 1
\end{array}\right)\left(\begin{array}{cc}
1 & 0 \\
\lambda_{0}+\lambda_{1} \frac{\mathrm{d}}{\mathrm{dt}} & \lambda_{2}
\end{array}\right)\left(\begin{array}{c}
\omega_{1} \\
\omega_{2}
\end{array}\right)
$$

where $\mu_{1} \lambda_{1}$ does not vanish. This is point (i).

(i) $\Rightarrow$ (ii): Let $\Omega_{1}, \Omega_{2}$ and $\Omega_{3}$ be defined by

$$
\begin{gathered}
\left(\begin{array}{l}
\Omega_{1} \\
\Omega_{2}
\end{array}\right)=J_{2}\left(\begin{array}{l}
\omega_{1} \\
\omega_{2}
\end{array}\right), \\
\left(\begin{array}{l}
\Omega_{3} \\
\Omega_{2}
\end{array}\right)=\left(\begin{array}{cc}
1 & -a \frac{\mathrm{d}}{\mathrm{dt}} \\
0 & 1
\end{array}\right)\left(\begin{array}{l}
\Omega_{1} \\
\Omega_{2}
\end{array}\right)=\left(\begin{array}{c}
\Omega_{1}-a \dot{\Omega}_{2} \\
\Omega_{2}
\end{array}\right) .
\end{gathered}
$$

then (7.34) implies that $\left\{\Omega_{3}, \Omega_{2}\right\}$ is integrable and hence, for some 1 -forms $\Gamma_{i, j}$,

$$
\begin{aligned}
& \mathrm{d} \Omega_{2}=\Omega_{2} \wedge \Gamma_{2,2}+\Omega_{3} \wedge \Gamma_{2,3}=\Omega_{1} \wedge \Gamma_{2,3}+\Omega_{2} \wedge \Gamma_{2,2}-a \dot{\Omega}_{2} \wedge \Gamma_{2,3} \\
& \mathrm{~d} \Omega_{3}=\Omega_{2} \wedge \Gamma_{3,2}+\Omega_{3} \wedge \Gamma_{3,3}=\Omega_{1} \wedge \Gamma_{2,3}+\Omega_{2} \wedge \Gamma_{2,2}-a \dot{\Omega}_{2} \wedge \Gamma_{2,3} .
\end{aligned}
$$

Taking the time-derivative of the second equation yields

$$
\begin{aligned}
\mathrm{d} \dot{\Omega}_{2}= & \Omega_{1} \wedge \dot{\Gamma}_{2,3}+\dot{\Omega}_{1} \wedge \Gamma_{2,3} \\
& +\Omega_{2} \wedge \dot{\Gamma}_{2,2}+\dot{\Omega}_{2} \wedge\left(\Gamma_{2,2}-a \dot{\Gamma}_{2,3}-\dot{a} \Gamma_{2,3}\right)-a \ddot{\Omega}_{2} \wedge \Gamma_{2,3}
\end{aligned}
$$

and finally, since $\mathrm{d} \Omega_{1}=\mathrm{d}\left(\Omega_{3}+a \dot{\Omega}_{2}\right)=\mathrm{d} \Omega_{3}+a \mathrm{~d} \dot{\Omega}_{2}-\dot{\Omega}_{2} \wedge \mathrm{d} a$,

$$
\begin{aligned}
\mathrm{d} \Omega_{1}= & \Omega_{1} \wedge\left(\Gamma_{2,3}+a \dot{\Gamma}_{2,3}\right)+\Omega_{2} \wedge\left(\Gamma_{2,2}+a \dot{\Gamma}_{2,2}\right) \\
& +a \dot{\Omega}_{1} \wedge \Gamma_{2,3}+\dot{\Omega}_{2} \wedge\left(-a \Gamma_{2,3}+a \Gamma_{2,2}-a^{2} \dot{\Gamma}_{2,3}-a \dot{a} \Gamma_{2,3}-\mathrm{d} a\right) \\
& -a^{2} \ddot{\Omega}_{2} \wedge \Gamma_{2,3} \\
\mathrm{~d} \Omega_{2}= & \Omega_{1} \wedge \Gamma_{2,3}+\Omega_{2} \wedge \Gamma_{2,2}-a \dot{\Omega}_{2} \wedge \Gamma_{2,3} .
\end{aligned}
$$

From (7.36), $\left\{\Omega_{1}, \Omega_{2}\right\}$ is the same differential system as $\left\{\omega_{1}, \omega_{2}\right\}$ and therefore, from (7.31), d $\Omega_{i} \equiv \lambda_{i} \dot{\Omega}_{1} \wedge \dot{\Omega}_{2}$ modulo $\left\{\Omega_{1}, \Omega_{2}\right\}$ for $i=1,2$ and $\lambda_{i}$ certain functions; from the second equation in (7.40), this implies that $\Gamma_{2,3}$ is a linear combination of $\Omega_{1}, \Omega_{2}, \dot{\Omega}_{1}, \dot{\Omega}_{2}$; from the first equation in (7.40), it is actually a linear combination of $\Omega_{1}, \Omega_{2}, \dot{\Omega}_{2}$ because the $\dot{\Omega}_{1}$-term would produce a $\ddot{\Omega}_{2} \wedge \dot{\Omega}_{1}$-term in the last term of $\mathrm{d} \Omega_{1}$ (it cannot be canceled by another term because there is no $\ddot{\Omega}_{2}$ in $\gamma_{2,3}$ ); this implies, if $\Gamma_{2,3}=\lambda_{1} \Omega_{1}+\lambda_{1} \Omega_{2}+\lambda_{0} \dot{\Omega}_{2}$,

$$
\mathrm{d} \Omega_{2}=\Omega_{2} \wedge \widetilde{\Gamma}_{2,2}+\left(\lambda_{0}+a \lambda_{1}\right) \Omega_{1} \wedge \dot{\Omega}_{2}
$$

where $\widetilde{\Gamma}_{2,2}$ contains $\Gamma_{2,2}$ plus other terms. This implies in particular that $\mathrm{d} \Omega_{2} \equiv 0$ modulo $\left\{\Omega_{1}, \Omega_{2}\right\}$ which implies that $\Omega_{2}$ is in the first derived system of $\left\{\Omega_{1}, \Omega_{2}\right\}$ (i.e. in the annihilator of $\left\{X_{1}, X_{2},\left[X_{1}, X_{2}\right]\right\}$ ) and therefore that it is collinear to $\omega_{1}$, or in other terms that matrix $J_{2}$ is triangular:

$$
\left(\begin{array}{l}
\Omega_{1} \\
\Omega_{2}
\end{array}\right)=\left(\begin{array}{cc}
\beta_{1} & \beta_{2} \\
\alpha & 0
\end{array}\right)\left(\begin{array}{l}
\omega_{1} \\
\omega_{2}
\end{array}\right)
$$


where $\alpha \beta_{2}$ does not vanish. Then (7.31) yields

$$
\mathrm{d} \Omega_{2} \equiv \frac{1}{\beta_{2}} \Omega_{1} \wedge\left(\left(\delta_{1}-\frac{\beta_{1} \delta_{2}}{\beta_{2}}\right) \dot{\Omega}_{2}+\delta_{2} \frac{\alpha}{\beta_{2}} \dot{\Omega}_{1}\right) \quad \text { modulo } \Omega_{2} .
$$

By comparing this and (7.41), we see that $\delta_{2} \alpha=0$ which implies that $\delta_{2}$ is identically zero because $\alpha$ does not vanish.

\subsection{Proof of the Results on $(x, u)$-Dynamic Linearizability}

In this section, we prove theorem 4.2 and proposition 4.5. They are proved together because we are not able to prove the intrinsic condition of theorem 4.2 without the help of the coordinates of the normal form (4.13). In the course of the proof, we will need the four following technical lemmae (lemmae 7.6, 7.7, 7.8 and 7.9), that are proved further.

Lemma 7.6. Let $\omega_{1}$ and $\omega_{2}$ be chosen according to (4.6). Let $b$ and $J_{2}$ be respectively a scalar smooth function and a $2 \times 2$ invertible matrix (of degree zero) with entries smooth functions, defined on a neighborhood of a point $\mathcal{Y}$ and let $\Omega_{1}$ and $\Omega_{3}$ be defined by

$$
\left(\begin{array}{l}
\Omega_{1} \\
\Omega_{3}
\end{array}\right)=\left(\begin{array}{cc}
1 & 0 \\
-b \frac{\mathrm{d}}{\mathrm{dt}} & 1
\end{array}\right) J_{2}\left(\begin{array}{l}
\omega_{1} \\
\omega_{2}
\end{array}\right) .
$$

The forms $\Omega_{1}$ and $\Omega_{3}$ satisfy the following relations:

$$
\begin{aligned}
& \mathrm{d} \Omega_{1} \equiv 0 \quad \text { modulo }\left\{\Omega_{1}, \Omega_{3}, \dot{\Omega}_{3}\right\} \\
& \mathrm{d} \Omega_{3} \equiv 0 \quad \text { modulo }\left\{\Omega_{3}, \Omega_{1} \wedge \dot{\Omega}_{3}\right\}
\end{aligned}
$$

on a neighborhood of $\mathcal{Y}$ if and only if there exist smooth functions $h_{1}, h_{2}$ and $a$, and $a 2 \times 2$ invertible matrix $J_{1}$ with entries smooth functions defined on a neighborhood of $\mathcal{Y}$, such that

$$
\begin{aligned}
\left(\begin{array}{l}
\mathrm{d} h_{1} \\
\mathrm{~d} h_{2}
\end{array}\right) & =J_{1}\left(\begin{array}{cc}
1 & -a \frac{\mathrm{d}}{\mathrm{dt}} \\
0 & 1
\end{array}\right)\left(\begin{array}{cc}
1 & 0 \\
-b \frac{\mathrm{d}}{\mathrm{dt}} & 1
\end{array}\right) J_{2}\left(\begin{array}{l}
\omega_{1} \\
\omega_{2}
\end{array}\right) \\
& =J_{1}\left(\begin{array}{cc}
1 & -a \frac{\mathrm{d}}{\mathrm{dt}} \\
0 & 1
\end{array}\right)\left(\begin{array}{l}
\Omega_{1} \\
\Omega_{3}
\end{array}\right) .
\end{aligned}
$$

LEMMA 7.7. Let $\omega_{1}$ and $\omega_{2}$ be some 1-forms satisfying (4.6), and hence (4.8), around a point where $\gamma$ and $\delta_{1,2}^{2}$ do not vanish (implied by the rank assumptions (4.1)-(4.2)-(4.3)-(4.4)-(4.5)).

(i) There cannot exist functions $a, b, h_{1}, h_{2}$ and two invertible $2 \times 2$ matrices of degree zero $J_{1}$ and $J_{2}$, all defined on a neighborhood of the considered point, such that

$$
J_{1}\left(\begin{array}{cc}
1 & 0 \\
-a \frac{\mathrm{d}}{\mathrm{dt}}^{2} b \frac{\mathrm{d}}{\mathrm{dt}}^{2} & 1
\end{array}\right) J_{2}\left(\begin{array}{l}
\omega_{1} \\
\omega_{2}
\end{array}\right)=\left(\begin{array}{c}
\mathrm{d} h_{1} \\
\mathrm{~d} h_{2}
\end{array}\right) .
$$

(ii) There cannot exist functions $\alpha, a, b, h_{1}, h_{2}$ and an invertible $2 \times 2$ matrix of degree zero $J_{1}$, all defined on a neighborhood of the considered point, such that

$$
J_{1}\left(\begin{array}{cc}
1 & -a \frac{\mathrm{d}}{\mathrm{dt}} \\
0 & 1
\end{array}\right)\left(\begin{array}{cc}
1 & 0 \\
-b \frac{\mathrm{d}}{\mathrm{dt}} & 1
\end{array}\right)\left(\begin{array}{ll}
1 & 0 \\
\alpha & 1
\end{array}\right)\left(\begin{array}{l}
\omega_{1} \\
\omega_{2}
\end{array}\right)=\left(\begin{array}{c}
\mathrm{d} h_{1} \\
\mathrm{~d} h_{2}
\end{array}\right) .
$$


LEMMA 7.8. Let $\omega_{1}$ and $\omega_{2}$ be some 1-forms satisfying (4.6), and hence (4.8). If for some functions $\lambda_{1}, \lambda_{2}$ and $\lambda_{3}$, one has

$$
\begin{aligned}
\mathrm{d} \omega_{2} & \equiv 0 \text { modulo }\left\{\omega_{2}, \Omega, \dot{\Omega}\right\} \\
\text { with } \Omega & =\lambda_{1} \omega_{1}+\lambda_{2} \omega_{2}+\lambda_{3} \dot{\omega}_{2},
\end{aligned}
$$

then $\lambda_{1}$ and $\lambda_{3}$ are related to the functions appearing in (4.8) by:

$$
\lambda_{3}\left(2 \gamma \lambda_{1}+\delta_{2,1}^{1} \lambda_{3}\right)=0 .
$$

LEMMA 7.9. Let $f$ and $g$ be two smooth functions from an open subset $O \subset$ $\mathbb{R}^{4}$ to $\mathbb{R}$. The following two assertions are equivalent:

(i) $\frac{\partial g}{\partial z_{4}}$ does not vanish on $O$ and $f$ and $g$ are solutions of the following equations on $O$ :

$$
\begin{aligned}
& 2 \frac{\partial g}{\partial z_{4}} \frac{\partial^{3} g}{\partial z_{4}^{3}}-3\left(\frac{\partial^{2} g}{\partial z_{4}^{2}}\right)^{2}=0 \\
& 2 \frac{\partial g}{\partial z_{4}} \frac{\partial^{3} f}{\partial z_{4}^{3}}-3 \frac{\partial^{2} g}{\partial z_{4}^{2}} \frac{\partial^{2} f}{\partial z_{4}^{2}}=0
\end{aligned}
$$

(ii) There exists $a_{0}, a_{1}, a_{2}, b_{0}, b_{1}, c_{0}$ and $c_{1}$, seven smooth functions of $z_{1}, z_{2}, z_{3}$ defined on $O$ (i.e. on its projection on $\mathbb{R}^{3}$ ) such that

$$
c_{0}\left(z_{1}, z_{2}, z_{3}\right)+z_{4} c_{1}\left(z_{1}, z_{2}, z_{3}\right) \quad \text { and } \quad\left|\begin{array}{ll}
b_{0} & b_{1} \\
c_{0} & c_{1}
\end{array}\right|\left(z_{1}, z_{2}, z_{3}\right)
$$

do not vanish on $O$ and $f$ and $g$ are given by (4.16) on $O$.

Proof of theorem 3.1 and Proposition 4.5. Let us consider a point $\overline{\mathcal{X}}=(\bar{x}, \bar{u}, \overline{\bar{u}}, \ldots)$ such that conditions (4.1)-(4.2)-(4.3)-(4.4)-(4.5) hold at $(\bar{x}, \bar{u})$. Let the forms $\omega_{1}$ and $\omega_{2}$ be defined according to (4.6) and the functions $\delta_{2,1}^{1}$ and $\gamma$ by (4.8). Let also $\left(z_{1}, z_{2}, z_{3}, z_{4}\right)$ be some coordinates in which system (1.5) has the form (4.13) (they exist from Proposition 4.4), and the functions $f$ and $g$ be defined accordingly from an open subset of $\mathbb{R}^{4}$ to $\mathbb{R}$.

We have to prove the following:

1. The following three properties are equivalent:

- $(x, u)$-dynamic linearizability of system (1.5) at point $\overline{\mathcal{X}}$, or of (4.13) at the corresponding point in terms of $(z, v, \dot{v}, \ldots)$,

- conditions of proposition 4.5 on the functions $f$ and $g$,

- condition in terms of Pfaffian systems of theorem 4.2.

2. When they are satisfied, the possible pairs of linearizing outputs depending on $x$ and $u$ are these described in theorem 4.2 .

The (easy) proof of the second point will be given at the very end when equivalence is totally understood.

From proposition $2.12,(x, u)$-dynamic linearizability is equivalent to existence of a matrix $P\left(\frac{\mathrm{d}}{\mathrm{dt}}\right)$ whose entries are polynomials in $\frac{\mathrm{d}}{\mathrm{dt}}$ of degree at most 2, which has an inverse of the same type (except we do not need to know whether the degree of the entries of the inverse is also at most 2 ), and transforms the pair of forms $\left(\omega_{1}, \omega_{2}\right)$ into a pair that defines an integrable Pfaffian system. Now use the second point of proposition 2.13; it allows four possible decompositions of the matrix $P\left(\frac{\mathrm{d}}{\mathrm{dt}}\right)$, but only on an open dense set of points of the neighborhood of $\overline{\mathcal{X}}$ where $P\left(\frac{\mathrm{d}}{\mathrm{dt}}\right)$ is defined, and $\overline{\mathcal{X}}$ might Esaim: CoCv, June 1997, Vol. 2, Pp. 151-230 
not belong to this open dense set. Around these points though, lemma 7.7 states that three of the four decompositions proposed by proposition 2.13 are impossible due to the form of $d \omega_{1}$ and $d \omega_{2}$ given by (4.7)-(4.8), so that only the last one is possible. If the decomposition of proposition 2.13 was available at all points, item 1 of the following lemma would be equivalent to $(x, u)$-dynamic linearizability, and the following lemma would end the proof.

LEMMA 7.10. Let $\omega_{1}$ and $\omega_{2}$ be chosen according to (4.6), the functions $\delta_{2,1}^{1}$ and $\gamma$ be defined by (4.8), and some coordinates $z_{1}, z_{2}, z_{3}, z_{4}$ be fixed according to Proposition 4.4, in which system (1.5) has the form (4.13), and the functions $f$ and $g$ be defined accordingly from an open subset of $\mathbb{R}^{4}$ to $\mathbb{R}$. The following four assertions are equivalent:

1. There exists an invertible matrix $J_{1}$ of degree zero and six functions $\alpha$, $\lambda, a, b, h_{1}$ and $h_{2}$, all defined on a neighborhood of the point $\mathcal{Y}$, such that $b$ does not vanish on this neighborhood and

$$
\begin{gathered}
\left(\begin{array}{l}
\mathrm{d} h_{1} \\
\mathrm{~d} h_{2}
\end{array}\right)=J_{1}\left(\begin{array}{cc}
1 & -a \frac{\mathrm{d}}{\mathrm{dt}} \\
0 & 1
\end{array}\right)\left(\begin{array}{cc}
1 & 0 \\
-b \frac{\mathrm{d}}{\mathrm{dt}} & 1
\end{array}\right)\left(\begin{array}{ll}
1 & 0 \\
\alpha & 1
\end{array}\right) \\
\times\left(\begin{array}{ll}
\lambda & 1 \\
1 & 0
\end{array}\right)\left(\begin{array}{l}
\omega_{1} \\
\omega_{2}
\end{array}\right) .
\end{gathered}
$$

2. There exist three functions $\alpha, \lambda$ and $b$, all defined on a neighborhood of $\mathcal{Y}$, such that $b$ does not vanish on this neighborhood and, with

$\left(\begin{array}{l}\Omega_{1} \\ \Omega_{3}\end{array}\right)=\left(\begin{array}{cc}1 & 0 \\ -b \frac{\mathrm{d}}{\mathrm{dt}} & 1\end{array}\right)\left(\begin{array}{ll}1 & 0 \\ \alpha & 1\end{array}\right)\left(\begin{array}{ll}\lambda & 1 \\ 1 & 0\end{array}\right)\left(\begin{array}{l}\omega_{1} \\ \omega_{2}\end{array}\right)$

one has

$$
\begin{array}{ll}
\mathrm{d} \Omega_{1} \equiv 0 & \text { modulo }\left\{\Omega_{1}, \Omega_{3}, \dot{\Omega}_{3}\right\} \\
\mathrm{d} \Omega_{3} \equiv 0 & \text { modulo }\left\{\Omega_{3}, \Omega_{1} \wedge \dot{\Omega}_{3}\right\} .
\end{array}
$$

3. $\delta_{2,1}^{1}$ does not vanish at $\mathcal{Y}$ and the first derived system of the Pfaffian system $\left\{\omega_{1}-\frac{2 \gamma}{\delta_{2,1}^{1}} \dot{\omega}_{2}, \omega_{2}\right\}$ has rank 1 and is integrable, i.e. there exists a (unique) function $\alpha$ such that (4.11) is satisfied.

4. The function $\delta_{2,1}^{1}$ does not vanish at $\mathcal{Y}$ and, in the normal form (4.13), the functions $f$ and $g$ are, on a neighborhood of $\mathcal{Y}$, of the form (4.16) where $a_{0}, a_{1}, a_{2}, b_{0}, b_{1}, c_{0}$ and $c_{1}$ are functions of $z_{1}, z_{2}, z_{3}$ only, which satisfy (4.17).

If one of these conditions is met (and therefore all of them), $\lambda, \alpha$ and $b$ in (7.54) and (7.55) are uniquely defined:

$$
\lambda=0, \quad b=\frac{2 \gamma}{\delta_{2,1}^{1}}, \quad \alpha \text { is uniquely defined by (4.11). }
$$

This lemma contains the real technical difficulties of the paper. The proof is given further (page 204), let us however sketch it. Equivalence between 1 and 2 is given by lemma 7.6, it is a manipulation on Pfaffian systems, and only needs the fact that the Pfaffian system $\left\{\omega_{1}, \omega_{2}\right\}$ may be written in four variables (the coordinates of $x$ ). It is very simple to prove that 3 implies 2, but the converse is not obvious: since we were not able to prove it directly, we used the coordinates $z$ of the normal form, and instead 
of proving that 2 implies 3 , we prove that 2 implies 4 by writing (7.56)(7.57) in the coordinates $(z, v, \dot{v}, \ldots)$ of the normal form (4.13) as some differential relations on the functions $\alpha, \lambda$ and $b$ with the functions $f$ and $g$ as parameters, eliminating the unknowns $\alpha, \lambda$ and $b$, and obtaining some PDEs on $f$ and $g$ that imply the form of $f$ and $g$ given by point 4 above (or by proposition 4.5), these computations have been conducted with the computer algebra system "Maple" (version 5.2). The fact that 4 implies 2 is a simple computation in coordinates, made easier by proposition 4.8 .

Unfortunately, the conclusion of proposition 2.13 is not valid at all point, so that the results we want to prove do not follow from the above lemma 7.10, proposition 2.13 and lemma 7.7 . Let us however prove that $(x, u)$ dynamic linearizability at point $\overline{\mathcal{X}}$ is equivalent to one of the four equivalent conditions of lemma 7.10 being satisfied at point $\overline{\mathcal{X}}$. This will end the proof that $(x, u)$-dynamic linearizability, the conditions of proposition 4.5 on the functions $f$ and $g$ (item 4 of lemma 7.10) and the condition in terms of Pfaffian systems of theorem 4.2 (item 3 of lemma 7.10) are equivalent.

Point 1 of lemma 7.10 implies $(x, u)$-dynamic linearizability from proposition 2.12 because the matrix applied to $\left(\omega_{1}, \omega_{2}\right)$ in $(7.54)$ is obviously invertible and of degree 2. Conversely, suppose that there exists a pair of linearizing outputs $\left(h_{1}, h_{2}\right)$ depending only on $x$ and $u$, defined around $\overline{\mathcal{X}}$, and let us prove that item 3 of lemma 7.10 holds on a neighborhood of $\overline{\mathcal{X}}$. From proposition 2.12, there exists $P\left(\frac{\mathrm{d}}{\mathrm{dt}}\right) \in \mathcal{A}(U)$, with $U$ a neighborhood $\overline{\mathcal{X}}$, such that

$$
\left\{\begin{array}{l}
P\left(\frac{\mathrm{d}}{\mathrm{dt}}\right) \text { is invertible in } \mathcal{A}(U) \\
\operatorname{deg} P \leq 2 \text { on } U \\
P\left(\frac{\mathrm{d}}{\mathrm{dt}}\right)\left(\begin{array}{l}
\omega_{1} \\
\omega_{2}
\end{array}\right)=\left(\begin{array}{c}
\mathrm{d} h_{1} \\
\mathrm{~d} h_{2}
\end{array}\right) .
\end{array}\right.
$$

Since $\gamma$ does not vanish at $\overline{\mathcal{X}}$ and the rank assumptions (4.1)-(4.2)-(4.3)(4.4)-(4.5) hold at $\overline{\mathcal{X}}$, we may suppose, by possibly restricting $U$, that

$$
\left\{\begin{array}{l}
\gamma \text { does not vanish on } U, \\
(4.1)-(4.2)-(4.3)-(4.4)-(4.5) \text { hold on } U, \\
\operatorname{deg} P=2 \text { on an open dense subset of } U .
\end{array}\right.
$$

The last statement is implied by the second one because if $\operatorname{deg} P$ is strictly less than 2 on an open set, the system is $x$-dynamic linearizable and this contradicts (4.4) from theorem 3.1.

Then, from proposition 2.13, there is an open dense subset $U_{0}$ of $U$ such that, for all $\mathcal{Y} \in U_{0}$, the matrix $P\left(\frac{\mathrm{d}}{\mathrm{dt}}\right)$ may be decomposed according to one of the four forms (2.13)-(2.14)-(2.15). From lemma 7.7 three of these four forms are forbidden, because conditions (4.1)-(4.2)-(4.3)-(4.4)-(4.5) hold at point $\mathcal{Y}$. Hence, around each point $\mathcal{Y} \in U_{0}$, there exists functions $\alpha, \lambda$, $a, b$, and a matrix $J_{1}$, defined on a neighborhood of $\mathcal{Y}$ such that (7.54) is true on a neighborhood of $\mathcal{Y}$. By restricting possibly the open sense set $U_{0}$, we may suppose that $b$ does not vanish on $U_{0}$ ( $b$ cannot vanish on an open set, because then $P$ would have degree at most 1 on this open set, and therefore the linearizing outputs would depend on $x$ only, and this would, from theorem 3.1, contradict (4.4). Then the conditions of point 1 of lemma Esaim: Cocv, June 1997, Vol. 2, PP. 151-230 
7.10 are satisfied on $U_{0}$. By applying lemma 7.10 at each point $\mathcal{Y}$ in $U_{0}$, one has, for all $\mathcal{Y} \in U_{0}$, a neighborhood of $\mathcal{Y}$ such that

- $\delta_{2,1}^{1}$ does not vanish on this neighborhood,

- there is a unique function $\alpha y$ defined on this neighborhood such that

$\mathrm{d}\left(\omega_{1}+\alpha y \omega_{2}-\frac{2 \gamma}{\delta_{2,1}^{1}} \dot{\omega}_{2}\right) \wedge\left(\omega_{1}+\alpha y \omega_{2}-\frac{2 \gamma}{\delta_{2,1}^{1}} \dot{\omega}_{2}\right)=0$

- there are a smooth scalar function $a_{\mathcal{y}}$ and an invertible matrix $J_{1, \mathcal{Y}}$ with entries some smooth functions, all defined on this neighborhood, so that, on this neighborhood,

$$
\left(\begin{array}{l}
\mathrm{d} h_{1} \\
\mathrm{~d} h_{2}
\end{array}\right)=J_{1, \mathcal{Y}}\left(\begin{array}{cc}
1 & -a_{\mathcal{y}} \frac{\mathrm{d}}{\mathrm{dt}} \\
0 & 1
\end{array}\right)\left(\begin{array}{cc}
1 & 0 \\
-\frac{2 \gamma}{\delta_{2,1}^{1}} \frac{\mathrm{d}}{\mathrm{dt}} & 1
\end{array}\right)\left(\begin{array}{cc}
0 & 1 \\
1 & \alpha \mathcal{y}
\end{array}\right)\left(\begin{array}{l}
\omega_{1} \\
\omega_{2}
\end{array}\right) \text {. }
$$

The last point is obtained by substituting the functions $\lambda$ and $b$ by the value they must have from (7.58). The second point implies in particular, by making the wedge product of both sides by $\omega_{2}$ and multiplying by $\left(\delta_{2,1}^{1}\right)^{2}$, that

$$
\begin{aligned}
\mathrm{d}\left(\delta_{2,1}^{1} \omega_{1}-2 \gamma \dot{\omega}_{2}\right) & \wedge\left(\delta_{2,1}^{1} \omega_{1}-2 \gamma \dot{\omega}_{2}\right) \wedge \omega_{2} \\
+\alpha \mathcal{y} \delta_{2,1}^{1} \mathrm{~d} \omega_{2} & \wedge\left(\delta_{2,1}^{1} \omega_{1}-2 \gamma \dot{\omega}_{2}\right) \wedge \omega_{2}=0 .
\end{aligned}
$$

but on the other hand, the differential form of degree $4 \mathrm{~d} \omega_{2} \wedge\left(\delta_{2,1}^{1} \omega_{1}-2 \gamma \dot{\omega}_{2}\right)$ $\wedge \omega_{2}$ is, from (4.8), given by

$$
\mathrm{d} \omega_{2} \wedge \omega_{2} \wedge\left(\delta_{2,1}^{1} \omega_{1}-2 \gamma \dot{\omega}_{2}\right)=\omega_{1} \wedge\left(\frac{1}{2} \delta_{2,1}^{1} \dot{\omega}_{1}-\gamma \ddot{\omega}_{2}\right) \wedge \omega_{2} \wedge\left(-2 \gamma \dot{\omega}_{2}\right),
$$

and therefore does not vanish on $U$. Existence of $\alpha y$ satisfying (7.63) may be translated in some determinants made with the coefficients of the two differential forms of degree 4 being zero, but if these determinants are zero on an open dense subset $U_{0}$, they are zero all over $U$, and therefore, since $\mathrm{d} \omega_{2} \wedge\left(\delta_{2,1}^{1} \omega_{1}-2 \gamma \dot{\omega}_{2}\right) \wedge \omega_{2}$ does not vanish, there is a function $\nu$, uniquely defined all over $U$, such that

$$
\begin{aligned}
\mathrm{d}\left(\delta_{2,1}^{1} \omega_{1}-2 \gamma \dot{\omega}_{2}\right) & \wedge\left(\delta_{2,1}^{1} \omega_{1}-2 \gamma \dot{\omega}_{2}\right) \wedge \omega_{2} \\
+\nu \mathrm{d} \omega_{2} & \wedge\left(\delta_{2,1}^{1} \omega_{1}-2 \gamma \dot{\omega}_{2}\right) \wedge \omega_{2}=0 .
\end{aligned}
$$

Of course, since on the neighborhood of each point $\mathcal{Y}$, the function $\alpha y$ is uniquely defined, it must coincide with $\frac{\nu}{\delta_{2,1}^{1}}$ where it is defined.

Then, let us define the form $\omega_{3}$ by

$$
\omega_{3}=\delta_{2,1}^{1} \omega_{1}+\nu \omega_{2}-2 \gamma \dot{\omega}_{2}
$$

equation (7.62) reads

$$
\left(\begin{array}{c}
\mathrm{d} h_{1} \\
\mathrm{~d} h_{2}
\end{array}\right)=J_{1, \mathcal{Y}}\left(\omega_{2}-a\left(\frac{1}{\delta_{2,1}^{1}} \dot{\omega}_{3}-\frac{\dot{\delta}_{2,1}^{1}}{\frac{1}{\delta_{2,1}^{1}} \omega_{3}} \omega_{3}\right)\right)
$$

and therefore, $\mathrm{d} h_{1}$ and $\mathrm{d} h_{2}$ are linear combinations of $\omega_{2}, \omega_{3}$ and $\dot{\omega}_{3}$ on a neighborhood of each point $\mathcal{Y} \in U_{0}$. This implies that the rank of $\left\{\mathrm{d} h_{1}, \mathrm{~d} h_{2}, \omega_{2}, \omega_{3}, \dot{\omega}_{3}\right\}$ is at most 3 on the open dense $U_{0}$, it is therefore also 
at most 3 on all $U$. Since the rank of $\left\{\omega_{2}, \omega_{3}, \dot{\omega}_{3}\right\}$ is three all over $U$ (because $\gamma$ does not vanish on $U$, see (7.60)), there are six functions $\mu_{i, j}$, (uniquely) defined all over $U$, such that

$$
\mathrm{d} h_{i}=\mu_{i, 1} \omega_{2}+\mu_{i, 2} \omega_{3}+\mu_{i, 3} \dot{\omega}_{3}
$$

for $i=1,2$, or in other words

$$
\left(\begin{array}{l}
\mathrm{d} h_{1} \\
\mathrm{~d} h_{2}
\end{array}\right)=\left(\begin{array}{ll}
\mu_{1,1} & \mu_{1,2}+\mu_{1,3} \frac{\mathrm{d}}{\mathrm{dt}} \\
\mu_{2,1} & \mu_{2,2}+\mu_{2,3} \frac{\mathrm{d}}{\mathrm{dt}}
\end{array}\right)\left(\begin{array}{c}
\omega_{2} \\
\omega_{3}
\end{array}\right)
$$

which implies, from (7.65),

$$
\left(\begin{array}{l}
\mathrm{d} h_{1} \\
\mathrm{~d} h_{2}
\end{array}\right)=\left(\begin{array}{ll}
\mu_{1,1} & \mu_{1,2}+\mu_{1,3} \frac{\mathrm{d}}{\mathrm{dt}} \\
\mu_{2,1} & \mu_{2,2}+\mu_{2,3} \frac{\mathrm{d}}{\mathrm{dt}}
\end{array}\right)\left(\begin{array}{cc}
0 & 1 \\
\delta_{2,1}^{1} & \nu-2 \gamma \frac{\mathrm{d}}{\mathrm{dt}}
\end{array}\right)\left(\begin{array}{l}
\omega_{1} \\
\omega_{2}
\end{array}\right)
$$

which implies, from (7.59), and because $\omega_{1}, \omega_{2}$ and all their time-derivatives are linearly independent, that

$$
P\left(\frac{\mathrm{d}}{\mathrm{dt}}\right)=\left(\begin{array}{ll}
\mu_{1,1} & \mu_{1,2}+\mu_{1,3} \frac{\mathrm{d}}{\mathrm{dt}} \\
\mu_{2,1} & \mu_{2,2}+\mu_{2,3} \frac{\mathrm{d}}{\mathrm{dt}}
\end{array}\right)\left(\begin{array}{cc}
0 & 1 \\
\delta_{2,1}^{1} & \nu-2 \gamma \frac{\mathrm{d}}{\mathrm{dt}}
\end{array}\right) .
$$

This implies that $\delta_{2,1}^{1}$ must not vanish on $U$ because $P\left(\frac{\mathrm{d}}{\mathrm{dt}}\right)$ could not be invertible in the neighborhood of the zeroes of $\delta_{2,1}^{1}$, where the first column of the second factor would vanish.

Since $\delta_{2,1}^{1}$ does not vanish on $U$, the function $\alpha \triangleq \frac{\nu}{\delta_{2,1}^{1}}$ is defined all over $U$, coincides with each $\alpha y$ where these are defined; this and (7.61) imply that (4.11) is satisfied on $U_{0}$ with this definition of $\alpha$; since $U_{0}$ is dense in $U,(4.11)$ is even satisfied all over $U$. This proves that $(x, u)$-linearizability implies item 3 of lemma 7.10, and ends the proof of equivalence between $(x, u)$ dynamic linearizability, the conditions of proposition 4.5 on the functions $f$ and $g$ and the condition in terms of Pfaffian systems of theorem 4.2.

To end the proof of theorem 4.2 and proposition 4.5, there only remains to prove that the possible pairs of linearizing outputs depending on $x$ and $u$ only are these described in theorem 4.2 . We have proved above that an arbitrary pair of linearizing output has to satisfy (7.66) around all points $\mathcal{Y}$ in an open and dense subset of a neighborhood of $\overline{\mathcal{X}}$, with $\omega_{3}=\delta_{2,1}^{1} \Omega_{3}$ (compare (7.65), the fact that $\alpha=\nu / \delta_{2,1}^{1}$ as noticed just after (7.64), and the definition of $\Omega_{3}$ in theorem 4.2). This implies that $\mathrm{d} h_{1}$ and $\mathrm{d} h_{2}$ are two independent linear combinations of $\Omega_{3}$ and $\omega_{2}-a \dot{\Omega}_{3}$ for a certain function $a$. This is exactly the form of a pair of linearizing outputs described in theorem 4.2 .

We now prove the four technical lemmae (lemmae 7.6, 7.7, 7.8 and 7.9) and then proceed with the proof of lemma 7.10 that was the cornerstone of the above proof.

Proof of Lemma 7.6: Suppose that $\Omega_{1}$ and $\Omega_{3}$ satisfy the identities (7.45)(7.46) on a neighborhood of $\mathcal{Y}$. Then the Pfaffian system $\left\{\Omega_{1}, \Omega_{3}, \dot{\Omega}_{3}\right\}$ is completely integrable because (7.45)-(7.46) obviously imply that $\mathrm{d} \Omega_{1}$ and $\mathrm{d} \Omega_{3}$ are zero modulo $\left\{\Omega_{1}, \Omega_{3}, \dot{\Omega}_{3}\right\}$, and (7.46) implies that, for a certain 1form $\Gamma_{3}$ and a certain function $k, \mathrm{~d} \Omega_{3}=\Omega_{3} \wedge \Gamma_{3}+k \Omega_{1} \wedge \dot{\Omega}_{3}$, but taking the time-derivative of both sides yields

$$
\mathrm{d} \dot{\Omega}_{3}=\dot{\Omega}_{3} \wedge \Gamma_{3}+\Omega_{3} \wedge \dot{\Gamma}_{3}+\dot{k} \Omega_{1} \wedge \dot{\Omega}_{3}+k \dot{\Omega}_{1} \wedge \dot{\Omega}_{3}+k \Omega_{1} \wedge \ddot{\Omega}_{3}
$$

Esaim: Cocv, June 1997, Vol. 2, Pf. 151-230 
which obviously implies that $\mathrm{d} \dot{\Omega}_{3}$ is zero modulo $\left\{\Omega_{1}, \Omega_{3}, \dot{\Omega}_{3}\right\}$. Integrability of this Pfaffian system implies that there exists a function $h_{1}$ defined on a neighborhood of $\mathcal{Y}$ such that

$$
\mathrm{d} h_{1}=\lambda_{1} \Omega_{1}+\lambda_{2} \Omega_{3}+\lambda_{3} \dot{\Omega}_{3}
$$

with $\lambda_{1}, \lambda_{2}, \lambda_{3}$ some functions, $\lambda_{1}$ nonzero at $\mathcal{Y}$. Then $\left\{\Omega_{3}, \mathrm{~d} h_{1}\right\}$ is integrable because (7.46) implies that $\mathrm{d} \Omega_{3}$ is zero modulo $\left\{\Omega_{3}, \mathrm{~d} h_{1} \wedge \dot{\Omega}_{3}\right\}$, and hence modulo $\left\{\Omega_{3}, \mathrm{~d} h_{1}\right\}$. Hence there is a second function $h_{2}$ such that

$$
\mathrm{d} h_{2}=\mu_{1} \mathrm{~d} h_{1}+\mu_{2} \Omega_{3}
$$

with $\mu_{1}, \mu_{2}$ some functions, $\mu_{2}$ nonzero at $\mathcal{Y}$. The functions $h_{1}, h_{2}$ built above, together with $a=-\lambda_{3} / \lambda_{1}$ and $J_{1}=\left(\begin{array}{cc}1 & 0 \\ \mu_{1} & \mu_{2}\end{array}\right)\left(\begin{array}{cc}\lambda_{1} & \lambda_{2} \\ 0 & 1\end{array}\right)$ satisfy (7.47).

Conversely, suppose that (7.47) holds. Let us define $\Omega_{1}, \Omega_{2}, \Omega_{3}, \Omega_{4}$ by

$$
\begin{gathered}
\left(\begin{array}{l}
\Omega_{1} \\
\Omega_{2}
\end{array}\right)=J_{2}\left(\begin{array}{l}
\omega_{1} \\
\omega_{2}
\end{array}\right) \\
\left(\begin{array}{l}
\Omega_{4} \\
\Omega_{3}
\end{array}\right)=\left(\begin{array}{cc}
1 & -a \frac{\mathrm{d}}{\mathrm{dt}} \\
0 & 1
\end{array}\right)\left(\begin{array}{cc}
1 & 0 \\
-b \frac{\mathrm{d}}{\mathrm{dt}} & 1
\end{array}\right)\left(\begin{array}{l}
\Omega_{1} \\
\Omega_{2}
\end{array}\right) .
\end{gathered}
$$

i.e.

$$
\begin{aligned}
& \Omega_{3}=\Omega_{2}-b \dot{\Omega}_{1} \\
& \Omega_{4}=\Omega_{1}-a \dot{\Omega}_{3}=\Omega_{1}-a\left(\dot{\Omega}_{2}-\dot{b} \dot{\Omega}_{1}-b \ddot{\Omega}_{1}\right) .
\end{aligned}
$$

We shall use the following basis (over smooth functions) for the space of all 1-forms:

$$
\left\{\Omega_{1}, \Omega_{2}, \Omega_{3}, \dot{\Omega}_{2}, \Omega_{4}, \dot{\Omega}_{4}, \ddot{\Omega}_{2}, \Omega_{2}^{(3)}, \Omega_{2}^{(4)}, \ldots, \Omega_{1}^{(4)}, \Omega_{1}^{(5)}, \Omega_{1}^{(6)}, \ldots\right\}
$$

where, in addition, $\left\{\Omega_{1}, \Omega_{2}, \Omega_{3}, \dot{\Omega}_{2}\right\}$ is a basis of $\operatorname{Span}\{\mathrm{d} \mathbf{x}\}$.

Then (7.54) implies that the Pfaffian system $\left(\Omega_{3}, \Omega_{4}\right)$ is completely integrable and therefore that there exists some 1 -forms $\Gamma_{i, j}$ such that

$$
\left\{\begin{array}{l}
\mathrm{d} \Omega_{3}=\Omega_{3} \wedge \Gamma_{3,3}+\Omega_{4} \wedge \Gamma_{3,4} \\
\mathrm{~d} \Omega_{4}=\Omega_{3} \wedge \Gamma_{4,3}+\Omega_{4} \wedge \Gamma_{4,4}
\end{array}\right.
$$

It is possible to express the 1 -forms $\Gamma_{i, j}$ in (7.73) as (finite) linear combinations of the forms in (7.72), and it is always possible to choose them such that, for $i=3,4$,

$$
\left\{\begin{array}{l}
\Gamma_{i, 3} \text { has no } \Omega_{3} \text { term, } \\
\Gamma_{i, 4} \text { has no } \Omega_{3} \text { term and no } \Omega_{4} \text { term. }
\end{array}\right.
$$

Taking the exterior derivative of (7.71) yields

$$
\mathrm{d} \Omega_{1}=\mathrm{d} \Omega_{4}+a \mathrm{~d} \dot{\Omega}_{3}-\dot{\Omega}_{3} \wedge \mathrm{d} a,
$$

and taking the time-derivative of the first equation in (7.73) yields

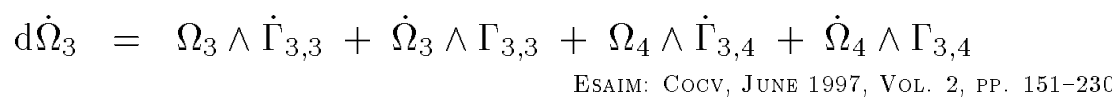


and finally, the two above equations yield, since $\dot{\Omega}_{3}=\frac{\Omega_{1}-\Omega_{4}}{a}$,

$$
\begin{aligned}
\mathrm{d} \Omega_{1}= & \Omega_{1} \wedge\left(\Gamma_{3,3}-\frac{\mathrm{d} a}{a}\right)+\Omega_{3} \wedge\left(\Gamma_{4,3}+a \dot{\Gamma}_{3,3}\right) \\
& +\Omega_{4} \wedge\left(\Gamma_{4,4}-\Gamma_{3,3}+a \dot{\Gamma}_{3,4}+\frac{\mathrm{d} a}{a}\right)+a \dot{\Omega}_{4} \wedge \Gamma_{3,4} \cdot(7.7
\end{aligned}
$$

On the other hand, since $\left(\Omega_{1}, \Omega_{2}\right)=\left(X_{1}, X_{2}\right)^{\perp}$, the Pfaffian system defined by $\left(\Omega_{1}, \Omega_{2}\right)$ can be defined with the help of the variable $x$ (i.e. the four coordinates of $x$ ) only, and therefore (see (8.3) in the Appendix), its Cartan characteristic system is at most $\operatorname{Span}\{\mathrm{d} \mathbf{x}\}$, i.e. at most $\left\{\Omega_{1}, \Omega_{2}, \Omega_{3}, \dot{\Omega}_{2}\right\}$, which implies that, for some functions $k_{1}$ and $k_{2}$,

$$
\left\{\begin{array}{l}
\mathrm{d} \Omega_{1} \equiv k_{1} \Omega_{3} \wedge \dot{\Omega}_{2} \operatorname{modulo}\left\{\Omega_{1}, \Omega_{2}\right\} \\
\mathrm{d} \Omega_{2} \equiv k_{2} \Omega_{3} \wedge \dot{\Omega}_{2} \operatorname{modulo}\left\{\Omega_{1}, \Omega_{2}\right\}
\end{array}\right.
$$

The first equation above implies, from (7.75) and (7.74), and using the fact that the 1-forms in $(7.72)$ are a basis for all 1-forms, that $\Gamma_{4,3}+a \dot{\Gamma}_{3,3}$ is a linear combination of $\Omega_{1}, \Omega_{2}, \Omega_{3}, \dot{\Omega}_{2}$ and $\Omega_{4}, \Gamma_{4,4}-\Gamma_{3,3}+a \dot{\Gamma}_{3,4}+\frac{\mathrm{d} a}{a}$ is a linear combination of $\Omega_{1}, \Omega_{2}, \Omega_{3}$ and $\Omega_{4}$, and $\Gamma_{3,4}$ is a linear combination of $\Omega_{1}, \Omega_{2}$ and $\dot{\Omega}_{4}$, with the coefficient of $\Omega_{4}$ in $\Gamma_{4,3}+a \dot{\Gamma}_{3,3}$ equal to the coefficient of $\Omega_{3}$ in $\Gamma_{4,4}-\Gamma_{3,3}+a \dot{\Gamma}_{3,4}+\frac{\mathrm{d} a}{a}$ :

$$
\begin{aligned}
\Gamma_{4,3}+a \dot{\Gamma}_{3,3} & =c_{1} \Omega_{1}+c_{2} \Omega_{2}+c_{3} \Omega_{3}+c_{4} \dot{\Omega}_{2}+d_{3} \Omega_{4} \\
\Gamma_{4,4}-\Gamma_{3,3}+a \dot{\Gamma}_{3,4}+\frac{\mathrm{d} a}{a} & =d_{1} \Omega_{1}+d_{2} \Omega_{2}+d_{3} \Omega_{3}+d_{4} \Omega_{4} \\
\Gamma_{3,4} & =e_{1} \Omega_{1}+e_{2} \Omega_{2}+e_{3} \dot{\Omega}_{4}
\end{aligned}
$$

and finally, (7.75) yields

$$
\mathrm{d} \Omega_{1}=\Omega_{1} \wedge \Delta_{1}+\Omega_{2} \wedge \Delta_{2}+c_{4} \Omega_{3} \wedge \dot{\Omega}_{2}
$$

with

$$
\left\{\begin{array}{l}
\Delta_{1}=\Gamma_{3,3}-\frac{\mathrm{d} a}{a}-c_{1} \Omega_{3}-d_{1} \Omega_{4}-a e_{1} \dot{\Omega}_{4} \\
\Delta_{2}=-c_{2} \Omega_{3}-d_{2} \Omega_{4}-a e_{2} \dot{\Omega}_{4} .
\end{array}\right.
$$

Now, from $(7.70)$,

$$
\mathrm{d} \Omega_{2}=\mathrm{d} \Omega_{3}+b \mathrm{~d} \dot{\Omega}_{1}+\mathrm{d} b \wedge \dot{\Omega}_{1},
$$

which allows, getting $\mathrm{d} \Omega_{3}$ from (7.73) and $\mathrm{d} \dot{\Omega}_{1}$ from (7.78)'s time-derivative, and using the fact that $\dot{\Omega}_{1}=\frac{\Omega_{2}-\Omega_{3}}{b}$ and $\dot{\Omega}_{3}=\frac{\Omega_{1}-\Omega_{4}}{a}$, to compute $\mathrm{d} \Omega_{2}$ and, forgetting the exterior products starting with $\Omega_{1}, \Omega_{2}$ or $\Omega_{3}$, to obtain

$$
\mathrm{d} \Omega_{2} \equiv b\left(\frac{c_{4}}{a}-d_{2}\right) \dot{\Omega}_{2} \wedge \Omega_{4}-a b e_{2} \dot{\Omega}_{2} \wedge \dot{\Omega}_{4}+e_{3} \Omega_{4} \wedge \dot{\Omega}_{4}
$$

modulo $\left\{\Omega_{1}, \Omega_{2}, \Omega_{3}\right\}$ which, since the second identity in (7.76) implies $\mathrm{d} \Omega_{2} \equiv$ 0 modulo $\left\{\Omega_{1}, \Omega_{2}, \Omega_{3}\right\}$, yields

$$
c_{4}=a d_{2} \text { and } e_{2}=e_{3}=0 .
$$

We get (7.45) from (7.78) with $e_{2}=0$ after substituting $\Omega_{4}$ for $\Omega_{1}-a \dot{\Omega}_{3}$. The same substitution in (7.71)-(7.77) with $e_{2}=e_{3}=0$ yields (7.46).

Proof of Lemma 7.7. (i) First, let us notice that $b$ cannot be identically zero around the considered point, because this would imply $x$-dynamic linearizability, which, from theorem 3.1, contradicts (4.4). Define $\Omega_{1}$ and $\Omega_{2}$ Esaim: Cocv, June 1997, Vol. 2, Pr. 151-230 
by $\left(\Omega_{1}, \Omega_{2}\right)^{T}=J_{2}\left(\omega_{1}, \omega_{2}\right)^{T}$; then (7.48) implies that the Pfaffian system $\left\{\Omega_{1}, \Omega_{2}-a \dot{\Omega}_{1}-b \ddot{\Omega}_{1}\right\}$ is completely integrable, which implies

$$
\mathrm{d} \Omega_{1}=\Omega_{1} \wedge \Gamma_{1}+\left(\Omega_{2}-a \dot{\Omega}_{1}-b \ddot{\Omega}_{1}\right) \wedge \Gamma_{2},
$$

for some 1 -forms $\Gamma_{1}$ and $\Gamma_{2}$. On the other hand, because $\left\{\Omega_{1}, \Omega_{2}\right\}$ span the annihilator of $\left\{X_{1}, X_{2}\right\}$, the characteristic system of this Pfaffian system is included in Span $\{\mathrm{dx}\}$ (see (8.3) in the Appendix), and hence one must have $\mathrm{d} \Omega_{1} \equiv k \eta_{1} \wedge \eta_{2}$ modulo $\left\{\Omega_{1}, \Omega_{2}\right\}$ with $k$ a function and $\eta_{1}$ and $\eta_{2}$ two form in $\operatorname{Span}\{d \mathbf{x}\}$. This implies, since $b$ does not vanish and $\ddot{\Omega}_{1}$ is not in $\operatorname{Span}\{\mathrm{d} \mathbf{x}\}$, that, in the above relation, $\Gamma_{2}$ is a linear combination of $\Omega_{1}, \Omega_{2}$ and $a \dot{\Omega}_{1}+b \ddot{\Omega}_{1}$, which in turn implies, for a certain function $k$,

$$
\mathrm{d} \Omega_{1} \equiv k \Omega_{2} \wedge\left(a \dot{\Omega}_{1}+b \ddot{\Omega}_{1}\right) \text { modulo } \Omega_{1} \text {. }
$$

This implies that $\Omega_{1}$ is in the derived system of the Pfaffian system $\left\{\Omega_{1}, \Omega_{2}\right\}$, and therefore, from (4.7)-(4.8), that $\Omega_{1}$ is collinear to $\omega_{1}$. The above relation with $\Omega_{1}$ collinear to $\omega_{1}$ contradicts $(4.7)$ because $a \dot{\Omega}_{1}+b \ddot{\Omega}_{1}$ is not a linear combination of $\omega_{1}, \omega_{2}$ and $\dot{\omega}_{2}$.

(ii) Suppose that (7.48) holds. From, lemma 7.6, the identities (7.45)-(7.46) must hold locally with

$$
\begin{aligned}
& \Omega_{1}=\omega_{1} \\
& \Omega_{3}=\omega_{2}+\alpha \omega_{1}-b \dot{\omega}_{1}
\end{aligned}
$$

and in particular, this would imply that

$$
\mathrm{d} \omega_{1} \equiv 0 \text { modulo }\left\{\omega_{1}, \omega_{2}-b \dot{\omega}_{1}, \dot{\omega}_{2}+(\alpha-\dot{b}) \dot{\omega}_{1}-b \ddot{\omega}_{1}\right\}
$$

which is impossible, because, from (4.7), $\mathrm{d} \omega_{1} \wedge \omega_{1} \wedge\left(\omega_{2}-b \dot{\omega}_{1}\right) \wedge\left(\dot{\omega}_{2}+(\alpha-\dot{b}) \dot{\omega}_{1}-b \ddot{\omega}_{1}\right)=b^{2} \delta_{1,2}^{2} \omega_{2} \wedge \dot{\omega}_{2} \wedge \omega_{1} \wedge \dot{\omega}_{1} \wedge \ddot{\omega}_{1}$.

Proof of Lemma 7.8. The expression for $\Omega$ in (7.50) implies

$$
\begin{aligned}
& \lambda_{1} \omega_{1}=\Omega \lambda_{2} \omega_{2}-\lambda_{3} \dot{\omega}_{2}, \\
& \lambda_{1} \dot{\omega}_{1}=-\dot{\lambda}_{1} \omega_{1}+\dot{\Omega}-\dot{\lambda}_{2} \omega_{2}-\left(\lambda_{3}+\lambda_{2}\right) \dot{\omega}_{2}-\lambda_{3} \ddot{\omega}_{2} .
\end{aligned}
$$

Using the above relations in (4.8), one obtains that $\lambda_{1}^{2} \mathrm{~d} \omega_{2}$ is equal to $\lambda_{3}\left(\delta_{2,1}^{1} \lambda_{3}+2 \gamma \lambda_{1}\right) \dot{\omega}_{2} \wedge \dot{\omega}_{2} \operatorname{modulo}\left\{\omega_{2}, \Omega, \dot{\Omega}\right\}$. This proves the lemma.

Proof of LEMMA 7.9. By simple substitution, it is clear that the forms of $f$ and $g$ given in (4.16) satisfy equations (7.52)-(7.53). Let us prove the converse. Since $\frac{\partial g}{\partial z_{4}} \neq 0$, one may define $h=1 / \frac{\partial g}{\partial z_{4}}$. Equation (7.52) then yields

$$
\left(\frac{\partial h}{\partial z_{4}}\right)^{2}-2 h \frac{\partial^{2} h}{\partial z_{4}^{2}}=0,
$$

whose non-vanishing solutions are exactly the squares, and opposite of squares of nonzero polynomials in $z_{4}$ of degree at most 1 , with coefficients function of $z_{1}, z_{2}$ and $z_{3}$; if the degree is $0, g$ is affine in $z_{4}$, if it is $1, g$ is homographic in $z_{4}$, still with coefficients function of $z_{1}, z_{2}$ and $z_{3}$, this yields the form for $g$ given in (4.16). Substituting $g$ for its expression given by (4.16) in equation (7.53) yields $\left(c_{0}+c_{1} z_{4}\right) \frac{\partial^{3} f}{\partial z_{4}^{3}}+3 c_{1} \frac{\partial^{2} f}{\partial z_{4}^{2}}=0$, which states that $\left(c_{0}+c_{1} z_{4}\right) f$ is a polynomial of degree at most 2 in $z_{4}$ and therefore implies that $f$ is of the form given in (4.16). 
Proof of lemma 7.10. $1 \Leftrightarrow 2$ : This is an obvious consequence of lemma 7.6 because (7.56)-(7.57) are identical to (7.45)-(7.46).

$\underline{3 \Rightarrow 2}$ : Let $b$ be defined by $(7.58)$ :

$$
b=\frac{2 \gamma}{\delta_{2,1}^{1}},
$$

and $\alpha$ be the one from relation (4.11). Define $\Omega_{1}$ and $\Omega_{3}$ as in (7.55), with $\lambda=0$. Relation (4.11) implies $\mathrm{d} \Omega_{3} \equiv 0 \operatorname{modulo} \Omega_{3}$, so it implies a fortiori (7.57). Now (7.56) is equivalent here to

$$
\mathrm{d} \omega_{2} \equiv 0 \quad \operatorname{modulo}\left\{\omega_{2}, \omega_{1}-b \dot{\omega}_{2}, \dot{\omega}_{1}+(\alpha-\dot{b}) \dot{\omega}_{2}-b \ddot{\omega}_{2}\right\}
$$

but a simple computation from (4.8) show that this is true when $b=\frac{2 \gamma}{\delta_{2,1}^{1}}$.

$\underline{4 \Rightarrow 3}$ : From proposition 4.8 , if point 4 is true, then some other coordinates may be found where the system has the simpler form (4.19). We shall compute in these coordinates with the following choice

$$
\begin{aligned}
& \omega_{1}=\mathrm{d} \zeta_{2}-\zeta_{3} \mathrm{~d} \zeta_{1} \\
& \omega_{2}=\mathrm{d} \zeta_{3}-\left(q_{0}+\zeta_{4} q_{1}\right) \mathrm{d} \zeta_{1}-\left(p_{1}+w_{1} q_{1}\right) \omega_{1} .
\end{aligned}
$$

On one hand, one has

$$
\mathrm{d} \omega_{2} \equiv 0 \operatorname{modulo}\left\{\omega_{2}, \mathrm{~d} \zeta_{1}, \mathrm{~d} w_{1}\right\}
$$

by computing the exterior derivative of $\omega_{2}$ given by (7.82) and replacing $\mathrm{d} \zeta_{1}$ and $\mathrm{d} w_{1}$ with zero and $\mathrm{d} \zeta_{3}$ with $\left(p_{1}+w_{1} q_{1}\right) \mathrm{d} \zeta_{2}$.

On the other hand, from (4.5), $\left\{\omega_{1}, \omega_{2}, \dot{\omega}_{2}\right\}$ is a basis of $\left\{\mathrm{d} \zeta_{1}, \mathrm{~d} \zeta_{2}, \mathrm{~d} \zeta_{3}\right\}$ and hence one has

$$
\mathrm{d} \zeta_{1}=\lambda_{1} \omega_{1}+\lambda_{2} \omega_{2}+\lambda_{3} \dot{\omega}_{2}
$$

for some functions $\lambda_{1}, \lambda_{2}$ and $\lambda_{3}$. Applying lemma 7.8 for $\Omega=\mathrm{d} \zeta_{1}$, and noticing that $\lambda_{3}$ cannot vanish because $\omega_{1} \wedge \omega_{2} \wedge \mathrm{d} \zeta_{1}$ does not vanish from (7.82) yields, from (7.83):

$$
2 \gamma \lambda_{1}+\delta_{2,1}^{1} \lambda_{3}=0
$$

The above two relation imply, since by assumption $\delta_{2,1}^{1}$ does not vanish, that $\mathrm{d} \zeta_{1}$ is a linear combination of $\omega_{2}$ and $\omega_{1}-\frac{2 \gamma}{\delta_{2,1}^{1}} \dot{\omega}_{2}$, and this clearly implies point 3 .

$2 \Rightarrow 4$ : This is the long and difficult part of the proof. It is all done using the symbolic computation system Maple, version 5, release 3 , with the package "liesymm" to manipulate differential forms, in the coordinates of the normal form (4.13).

We are now working in coordinates, with system (4.13) for some $f$ and $g$. We make the following choice for $\omega_{1}$ and $\omega_{2}$ :

$$
\begin{aligned}
& \omega_{1}=\mathrm{d} z_{2}-z_{3} \mathrm{~d} z_{1} \\
& \omega_{2}=\mathrm{d} z_{3}-g \mathrm{~d} z_{1}-\left(\frac{\partial f}{\partial z_{4}}+v_{1} \frac{\partial g}{\partial z_{4}}\right) \omega_{1} .
\end{aligned}
$$

The idea of the proof is quite straightforward: We suppose that there exists functions $\alpha, \lambda$ and $b$ satisfying (7.56)-(7.57), we write these equations explicitly in terms of $\alpha, \lambda$ and $b$, and we eliminate $\alpha, \lambda$ and $b$ to obtain the conditions on $f$ and $g$ are as described in point 4 . 
STEP 1. With the choice (7.84) for $\omega_{1}$ and $\omega_{2}$, we have the following decomposition of $d \omega_{1}$ and $d \omega_{2}$, more precise than (4.7) and (4.8) in proposition 4.1:

$$
\begin{aligned}
\mathrm{d} \omega_{1} & =\omega_{1} \wedge\left(\delta_{1,1}^{0} \omega_{2}+\delta_{1,1}^{2} \dot{\omega}_{2}\right)+\delta_{1,2}^{2} \omega_{2} \wedge \dot{\omega}_{2} \\
\mathrm{~d} \omega_{2} & =\omega_{1} \wedge\left(\delta_{2,1}^{0} \omega_{2}+\delta_{2,1}^{1} \dot{\omega}_{1}+\delta_{2,1}^{2} \dot{\omega}_{2}-\gamma \ddot{\omega}_{2}\right) \\
& +\omega_{2} \wedge\left(\delta_{2,2}^{1} \dot{\omega}_{1}+\delta_{2,2}^{2} \dot{\omega}_{2}\right) \gamma \dot{\omega}_{1} \wedge \dot{\omega}_{2}
\end{aligned}
$$

for some functions $\delta_{i, j}^{k}$ and $\gamma$ that may be computed explicitly using $f, g$ and some of their partial derivatives.

Indeed, (4.7) reads

$$
\mathrm{d} \omega_{1}=\omega_{1} \wedge \Gamma_{1}+\delta_{1,2}^{2} \omega_{2} \wedge \dot{\omega}_{2},
$$

for some form $\Gamma_{1}$, but $\mathrm{d} \omega_{1}=\mathrm{d} z_{1} \wedge \mathrm{d} z_{3}$ and $\left\{\omega_{1}, \omega_{2}, \dot{\omega}_{2}\right\}$ is a basis of $\left\{\mathrm{d} z_{1}, \mathrm{~d} z_{2}, \mathrm{~d} z_{3}\right\}$-because it is the characteristic system of $\left\{\omega_{1}\right\}$ from the above equation - so that $\Gamma_{1}$ must be a linear combination of $\omega_{1}, \omega_{2}$ and $\dot{\omega}_{2}$. This implies (7.85).

Also, (4.8) reads

$$
\mathrm{d} \omega_{2}=\omega_{2} \wedge \Gamma_{2}+\omega_{1} \wedge\left(\delta_{2,1}^{0} \omega_{2}+\delta_{2,1}^{1} \dot{\omega}_{1}+\delta_{2,1}^{2} \dot{\omega}_{2}-\gamma \ddot{\omega}_{2}\right)+\gamma \dot{\omega}_{1} \wedge \dot{\omega}_{2},
$$

for a certain form $\Gamma_{2}$, but

$$
\mathrm{d} \omega_{2}=\mathrm{d} z_{1} \wedge \mathrm{d} g-\left(\frac{\partial f}{\partial z_{4}}+v_{1} \frac{\partial g}{\partial z_{4}}\right) \mathrm{d} z_{1} \wedge \mathrm{d} z_{3}-\mathrm{d}\left(\frac{\partial f}{\partial z_{4}}+v_{1} \frac{\partial g}{\partial z_{4}}\right) \wedge \omega_{1}
$$

and hence $\mathrm{d} \omega_{2}$ is, modulo $\left\{\omega_{1}\right\}$, a linear combination of $\mathrm{d} z_{1}, \mathrm{~d} z_{2}, \mathrm{~d} z_{3}$ and $\mathrm{d} z_{4}$, i.e. of $\omega_{1}, \omega_{2}, \dot{\omega}_{1}$ and $\dot{\omega}_{2}$; this implies that $\Gamma_{2}$ must be a linear combination of $\omega_{1}, \omega_{2}, \dot{\omega}_{1}$ and $\dot{\omega}_{2}$, and therefore (7.86).

STEP 2. If $\alpha, \lambda$ and $b$ satisfy (7.56)-(7.57), then

$\lambda$ may depend on $z_{1}, z_{2}, z_{3}, z_{4}, v_{2}-v_{1} f\left(z_{1}, z_{2}, z_{3}, z_{4}\right)$ only, $\alpha$ and $b$ may depend on $z_{1}, z_{2}, z_{3}, z_{4}, v_{1}, v_{2}, \dot{v}_{1}, \dot{v}_{2}$ only.

Relations (7.68) and (7.70) imply:

$$
\begin{aligned}
\Omega_{1}= & \omega_{2}+\lambda \omega_{1} \\
\Omega_{2}= & \omega_{1}+\alpha \Omega_{1}=(1+\alpha \lambda) \omega_{1}+\alpha \omega_{2} \\
\Omega_{3}= & \omega_{1}+\alpha \Omega_{1}-b \dot{\Omega}_{1} \\
= & \alpha\left(\omega_{2}+\lambda \omega_{1}\right)-b\left(\dot{\omega}_{2}+\lambda \dot{\omega}_{1}+\left(\dot{\lambda}-\frac{1}{b}\right) \omega_{1}\right) \\
\dot{\Omega}_{3}= & (\dot{\lambda}(\alpha-\dot{b})-b \ddot{\lambda}) \omega_{1}+\dot{\alpha}\left(\omega_{2}+\lambda \omega_{1}\right) \\
& +(1-2 b \dot{\lambda}) \dot{\omega}_{1}+(\alpha-\dot{b})\left(\dot{\omega}_{2}+\lambda \dot{\omega}_{1}\right)-b\left(\ddot{\omega}_{2}+\lambda \ddot{\omega}_{1}\right)
\end{aligned}
$$

Taking the exterior derivative of (7.90) and (7.88) yields

$$
\begin{aligned}
& \mathrm{d} \Omega_{3}=\mathrm{d} \omega_{1}+\alpha \mathrm{d} \Omega_{1}-b \mathrm{~d} \dot{\Omega}_{1}+\mathrm{d} \alpha \wedge \Omega_{1}-\mathrm{d} b \wedge \dot{\Omega}_{1} \\
& \text { with } \mathrm{d} \Omega_{1}=\mathrm{d} \omega_{2}+\lambda \mathrm{d} \omega_{1}+\mathrm{d} \lambda \wedge \omega_{1} \\
& \mathrm{~d} \dot{\Omega}_{1}=\mathrm{d} \dot{\omega}_{2}+\dot{\lambda} \mathrm{d} \omega_{1}+\lambda \mathrm{d} \dot{\omega}_{1}+\mathrm{d} \dot{\lambda} \wedge \omega_{1}+\mathrm{d} \lambda \wedge \dot{\omega}_{1} \cdot(7.94)
\end{aligned}
$$


Relation (7.92) implies:

$$
\begin{aligned}
\mathrm{d} \Omega_{3}= & (1+\alpha \lambda-b \dot{\lambda}) \mathrm{d} \omega_{1}+\alpha \mathrm{d} \omega_{2}+\alpha \mathrm{d} \lambda \wedge \omega_{1} \\
& -b\left(\mathrm{~d} \dot{\omega}_{2}+\lambda \mathrm{d} \dot{\omega}_{1}+\mathrm{d} \dot{\lambda} \wedge \omega_{1}+\mathrm{d} \lambda \wedge \dot{\omega}_{1}\right) \\
& +\mathrm{d} \alpha \wedge\left(\omega_{2}+\lambda \omega_{1}\right)+\mathrm{d} b \wedge \frac{\Omega_{3}-\omega_{1}-\alpha\left(\omega_{2}+\lambda \omega_{1}\right)}{b} .
\end{aligned}
$$

Taking the time-derivative of both sides in (7.85) and (7.86), we have

$$
\left\{\begin{aligned}
\mathrm{d} \dot{\omega}_{1}= & \omega_{1} \wedge\left(\dot{\delta}_{1,1}^{0} \omega_{2}+\left(\delta_{1,1}^{0}+\dot{\delta}_{1,1}^{2}\right) \dot{\omega}_{2}+\delta_{1,1}^{2} \ddot{\omega}_{2}\right) \\
& +\omega_{2} \wedge\left(-\delta_{1,1}^{0} \dot{\omega}_{1}+\dot{\delta}_{1,2}^{2} \dot{\omega}_{2}+\delta_{1,2}^{2} \ddot{\omega}_{2}\right) \\
& +\delta_{1,1}^{2} \dot{\omega}_{1} \wedge \dot{\omega}_{2} \\
\mathrm{~d} \dot{\omega}_{2}= & \omega_{1} \wedge\left(\dot{\delta}_{2,1}^{0} \omega_{2}+\dot{\delta}_{2,1}^{1} \dot{\omega}_{1}+\left(\delta_{2,1}^{0}+\dot{\delta}_{2,1}^{2}\right) \dot{\omega}_{2}+\delta_{2,1}^{1} \ddot{\omega}_{1}\right. \\
& \left.+\left(\delta_{2,1}^{2}-\dot{\gamma}\right) \ddot{\omega}_{2}-\gamma \omega_{2}^{(3)}\right) \\
& +\omega_{2} \wedge\left(\left(\dot{\delta}_{2,2}^{1}-\delta_{2,1}^{0}\right) \dot{\omega}_{1}+\dot{\delta}_{2,2}^{2} \dot{\omega}_{2}+\delta_{2,2}^{1} \ddot{\omega}_{1}+\delta_{2,2}^{2} \ddot{\omega}_{2}\right) \\
& +\dot{\omega}_{2} \wedge\left(\left(-\delta_{2,1}^{2}+\delta_{2,2}^{1}-\dot{\gamma}\right) \dot{\omega}_{1}-\gamma \ddot{\omega}_{1}\right) .
\end{aligned}\right.
$$

Equation (7.57) implies in particular that $\mathrm{d} \Omega_{3} \equiv 0$ modulo $\left\{\omega_{1}, \Omega_{1}, \Omega_{3}\right\}$, i.e. - see $(7.90)-\operatorname{modulo}\left\{\omega_{1}, \omega_{2}, \dot{\omega}_{2}+\lambda \dot{\omega}_{1}\right\}$. Equations (7.85), (7.86) and (7.96) imply

$$
\left.\begin{array}{ll}
\mathrm{d} \omega_{1} \equiv 0 & \mathrm{~d} \dot{\omega}_{1} \equiv 0 \\
\mathrm{~d} \omega_{2} \equiv 0 & \mathrm{~d} \dot{\omega}_{2} \equiv \lambda \gamma \dot{\omega}_{1} \wedge \ddot{\omega}_{1}
\end{array}\right\} \operatorname{modulo}\left\{\omega_{1}, \omega_{2}, \dot{\omega}_{2}+\lambda \dot{\omega}_{1}\right\} .
$$

Then, from (7.92), (7.93), (7.94),

$$
\mathrm{d} \Omega_{3} \equiv-b\left(\lambda \gamma \dot{\omega}_{1} \wedge \ddot{\omega}_{1}+\mathrm{d} \lambda \wedge \dot{\omega}_{1}\right) \operatorname{modulo}\left\{\omega_{1}, \omega_{2}, \dot{\omega}_{2}+\lambda \dot{\omega}_{1}\right\}
$$

which in turn implies

$$
\mathrm{d} \lambda+\lambda \gamma \ddot{\omega}_{1} \equiv 0 \operatorname{modulo}\left\{\omega_{1}, \omega_{2}, \dot{\omega}_{1}, \dot{\omega}_{2}\right\} .
$$

Since $\left\{\mathrm{d} z_{1}, \mathrm{~d} z_{2}, \mathrm{~d} z_{3}, \mathrm{~d} z_{4}\right\}$ is a nother basis for $\left\{\omega_{1}, \omega_{2}, \dot{\omega}_{1}, \dot{\omega}_{2}\right\}$ and, from (7.84) and (4.13),

$$
\begin{aligned}
& \dot{\omega}_{1}=\mathrm{d} z_{4}+v_{1} \mathrm{~d} z_{3}-\left(f+v_{1} g\right) \mathrm{d} z_{1}, \\
& \ddot{\omega}_{1}=\mathrm{d}\left(v_{2}-f v_{1}\right)+v_{1} \mathrm{~d} f+\dot{v}_{1} \mathrm{~d} z_{3}+\left(\dot{f}+v_{1} \dot{g}+\dot{v}_{1} g\right) \mathrm{d} z_{1},
\end{aligned}
$$

that $\mathrm{d} \lambda$ is a linear combination of $\mathrm{d}\left(v_{2}-f v_{1}\right), \mathrm{d} z_{1}, \mathrm{~d} z_{2}, \mathrm{~d} z_{3}$ and $\mathrm{d} z_{4}$; this proves the statement on $\lambda$ in $(7.87)$.

Replacing $\dot{\omega}_{1}$ and $\ddot{\omega}_{1}$ with zero and $\dot{\omega}_{2}$ with $\left(\frac{1+\alpha \lambda}{b}-\dot{\lambda}\right) \omega_{1}+\frac{\alpha}{b} \omega_{2}$ in the expression of $\Omega_{1} \wedge \dot{\Omega}_{3}$ obtained from (7.88) and (7.91) obviously yields only some terms in $\omega_{1} \wedge \omega_{2}, \omega_{1} \wedge \ddot{\omega}_{2}$ and $\omega_{2} \wedge \ddot{\omega}_{2}$, hence

$$
\Omega_{1} \wedge \dot{\Omega}_{3} \equiv 0 \operatorname{modulo}\left\{\Omega_{3}, \dot{\omega}_{1}, \ddot{\omega}_{1}, \omega_{1} \wedge \omega_{2}, \omega_{1} \wedge \ddot{\omega}_{2}, \omega_{2} \wedge \ddot{\omega}_{2}\right\} \text {. }
$$

Therefore, Equation (7.57) implies in particular that $\mathrm{d} \Omega_{3} \equiv 0$ modulo $\left\{\Omega_{3}, \dot{\omega}_{1}, \ddot{\omega}_{1}, \omega_{1} \wedge \omega_{2}, \omega_{1} \wedge \ddot{\omega}_{2}, \omega_{2} \wedge \ddot{\omega}_{2}\right\}$, i.e. $\operatorname{modulo}\left\{b \dot{\omega}_{2}-\alpha\left(\omega_{2}+\lambda \omega_{1}\right)+\right.$ $\left.(b \dot{\lambda}-1) \omega_{1}, \dot{\omega}_{1}, \ddot{\omega}_{1}, \omega_{1} \wedge \omega_{2}, \omega_{1} \wedge \ddot{\omega}_{2}, \omega_{2} \wedge \ddot{\omega}_{2}\right\}$. From (7.85), (7.86) and (7.96), we have:

$$
\begin{array}{ll}
\mathrm{d} \omega_{1} \equiv 0 & \mathrm{~d} \dot{\omega}_{1} \equiv 0 \\
\mathrm{~d} \omega_{2} \equiv 0 & \mathrm{~d} \dot{\omega}_{2} \equiv-\gamma \omega_{1} \wedge \omega_{2}^{(3)}
\end{array}
$$


modulo $\left\{b \dot{\omega}_{2}-\alpha\left(\omega_{2}+\lambda \omega_{1}\right)+(b \dot{\lambda}-1) \omega_{1}, \dot{\omega}_{1}, \ddot{\omega}_{1}, \omega_{1} \wedge \omega_{2}, \omega_{1} \wedge \ddot{\omega}_{2}, \omega_{2} \wedge \ddot{\omega}_{2}\right\}$. Hence, from (7.92), (7.93), (7.94)

$$
\begin{aligned}
\mathrm{d} \Omega_{3} \equiv & \omega_{1} \wedge\left(b \gamma \omega_{2}^{(3)}-\alpha \mathrm{d} \lambda+b d \mathrm{~d} \dot{\lambda}+\frac{\mathrm{d} b}{b}\right) \\
& +\left(\omega_{2}+\lambda \omega_{1}\right) \wedge\left(\frac{\alpha}{b} \mathrm{~d} b-\mathrm{d} \alpha\right) .
\end{aligned}
$$

This implies in particular that

$$
\left.\begin{array}{rl}
b \gamma \omega_{2}^{(3)}-\alpha \mathrm{d} \lambda+b d \mathrm{~d} \dot{\lambda}+\frac{\mathrm{d} b}{b} & \equiv 0 \\
\frac{\alpha}{b} \mathrm{~d} b-\mathrm{d} \alpha & \equiv 0
\end{array}\right\} \operatorname{modulo}\left\{\omega_{1}, \omega_{2}, \dot{\omega}_{1}, \dot{\omega}_{2}, \ddot{\omega}_{1}, \ddot{\omega}_{2}\right\}
$$

We have already shown above that $\mathrm{d} \lambda$ is a linear combination of $\omega_{1}, \omega_{2}, \dot{\omega}_{1}$, $\dot{\omega}_{2}, \ddot{\omega}_{1}$; hence $\mathrm{d} \dot{\lambda}$ is a linear combination of $\omega_{1}, \omega_{2}, \dot{\omega}_{1}, \dot{\omega}_{2}, \ddot{\omega}_{1}, \ddot{\omega}_{2}, \omega_{1}^{(3)}$. This and the above equations imply that $\mathrm{d} b$ and $\mathrm{d} \alpha$ are linear combinations of $\omega_{1}, \omega_{2}, \dot{\omega}_{1}, \dot{\omega}_{2}, \ddot{\omega}_{1}, \ddot{\omega}_{2}, \omega_{1}^{(3)}, \omega_{2}^{(3)}$. This yields the second statement in (7.87) for $\left\{\omega_{1}, \omega_{2}, \dot{\omega}_{1}, \dot{\omega}_{2}, \ddot{\omega}_{1}, \ddot{\omega}_{2}, \omega_{1}^{(3)}, \omega_{2}^{(3)}\right\}$ is another basis for $\left\{\mathrm{d} z_{1}, \mathrm{~d} z_{2}, \mathrm{~d} z_{3}, \mathrm{~d} z_{4}\right.$, $\left.\mathrm{d} v_{1}, \mathrm{~d} v_{2}, \mathrm{~d} \dot{v}_{1}, \mathrm{~d} \dot{v}_{2}\right\}$.

Note that the second relation in (7.98) actually implies that $\alpha \mathrm{d} b-b \mathrm{~d} \alpha$ is a linear combination of $\omega_{1}, \omega_{2}, \dot{\omega}_{1}, \dot{\omega}_{2}, \ddot{\omega}_{1}, \ddot{\omega}_{2}$, i.e. that $\alpha / b$ depends on $z_{1}, z_{2}, z_{3}, z_{4}, v_{1}, v_{2}$ only (and not on $\dot{v}_{1}, \dot{v}_{2}$ ).

STEP 3. If $\alpha, \lambda$ and $b$ satisfy (7.56)-(7.57) with $b$ non vanishing, then $\lambda$ must be identically zero.

The core of this point is a rather heavy computation conducted in Maple. Let us explain some notations.

First of all, we need to work with a finite number of variable only. The only variables that will ever be needed during the computations are

$$
\left(z_{1}, z_{2}, z_{3}, z_{4}, v_{1}, v_{2}, \dot{v}_{1}, \dot{v}_{2}, \ddot{v}_{1}, \ddot{v}_{2}\right)
$$

because, the only operation that makes some new variables appear is taking the "time-derivative" of some objects. This occurs only when computing $\Omega_{1}, \Omega_{3}$ and $\dot{\Omega}_{3}$ according to (7.88), (7.90) and (7.91), but from (7.84), it is clear that the forms $\Omega_{1}, \Omega_{3}$ and $\dot{\Omega}_{3}$ may be expressed with the help of the above variables. This may be checked in the course of the computation: we set the time-derivative of $\ddot{v}_{1}$ and $\ddot{v}_{2}$ to "ERROR $R_{1}$ " and "ERROR $R_{2}$ " and we may check that we never have to apply the time-differentiation ("DOT") to $\ddot{v}_{1}$ and $\ddot{v}_{2}$ by checking that the variables "ERROR ${ }_{1}$ " and "ERROR $R_{2}$ " never appear in the expressions we compute.

To take advantage of the fact that $\lambda$ depends only on $z_{1}, z_{2}, z_{3}, z_{4}$ and $v_{2}-f v_{1}$, we make a change of coordinates, defining $w_{2}$ by

$$
w_{2}=v_{2}-f\left(z_{1}, z_{2} \cdot z_{3}, z_{4}\right) v_{2} \text {. }
$$

We work then in the coordinates

$$
\left(z_{1}, z_{2}, z_{3}, z_{4}, v_{1}, w_{2}, \dot{v}_{1}, \dot{v}_{2}, \ddot{v}_{1}, \ddot{v}_{2}\right)
$$


rather than the above, and the system is given by the derivative along its dynamics, i.e. along:

$$
\begin{aligned}
\frac{\mathrm{d}}{\mathrm{dt}} z_{1}= & v_{1} \\
\frac{\mathrm{d}}{\mathrm{dt}} z_{2}= & z_{4}+z_{3} v_{1} \\
\frac{\mathrm{d}}{\mathrm{dt}} z_{3}= & f\left(z_{1}, z_{2}, z_{3}, z_{4}\right)+g\left(z_{1}, z_{2}, z_{3}, z_{4}\right) v_{1} \\
\frac{\mathrm{d}}{\mathrm{dt}} z_{4}= & w_{2}+f\left(z_{1}, z_{2}, z_{3}, z_{4}\right) v_{1} \\
\frac{\mathrm{d}}{\mathrm{dt}} v_{1}= & \dot{v}_{1} \\
\frac{\mathrm{d}}{\mathrm{dt}} w_{2}= & \dot{v}_{2}-f\left(z_{1}, z_{2}, z_{3}, z_{4}\right) \dot{v}_{1} \\
& -u_{1}\left(\frac{\partial f}{\partial z_{1}} v_{1}+\frac{\partial f}{\partial z_{2}}\left(z_{4}+z_{3} v_{1}\right)+\frac{\partial f}{\partial z_{3}}\left(f+v_{1} g\right)+\frac{\partial f}{\partial z_{4}}\left(w_{2}+v_{1} f\right)\right) \\
\frac{\mathrm{d}}{\mathrm{dt}} \dot{v}_{1}= & \ddot{v}_{1} \\
\frac{\mathrm{d}}{\mathrm{dt}} \dot{v}_{2}= & \ddot{v}_{2} \\
\frac{\mathrm{d}}{\mathrm{dt}} \ddot{v}_{1}= & \operatorname{ERROR} R_{1} \\
\frac{\mathrm{d}}{\mathrm{dt}} \ddot{v}_{2}= & \text { ERROR }
\end{aligned}
$$

Since $b$ does not vanish, we may define new functions $\beta$ and $\rho$ from $b$ and $\alpha$ as follows:

$$
\begin{aligned}
b & =\frac{1}{\beta\left(z_{1}, z_{2}, z_{3}, z_{4}, v_{1}, w_{2}, \dot{v}_{1}, \dot{v}_{2}\right)} \\
\alpha & =\frac{\rho\left(z_{1}, z_{2}, z_{3}, z_{4}, v_{1}, w_{2}, \dot{v}_{1}, \dot{v}_{2}\right)}{\beta\left(z_{1}, z_{2}, z_{3}, z_{4}, v_{1}, w_{2}, \dot{v}_{1}, \dot{v}_{2}\right)} .
\end{aligned}
$$

Note that by assumption $b$-and therefore $\beta$ - does not vanish.

If $\lambda$ is not locally identically zero, then there are points arbitrarily close to the point under consideration where it does not vanish, and hence there are points where neither $\lambda$ nor $b$-and hence $\beta$ - vanish and the relations (7.56)-(7.57) hold.

In the following Maple session, we suppose we are at such a point, we write the equations for (7.56)-(7.57) in terms of the functions $\lambda, \beta$ and $\rho$, supposing that we may divide by $\lambda$ and by $\rho$ and get a contradiction (namely that $\lambda$ must be zero).

Remark 7.11. The symbol

$$
\&^{\wedge}
$$

in the Maple session stands for the exterior product (or wedge product).

\section{This reproduces a session run with Maple $V$ Release 3}

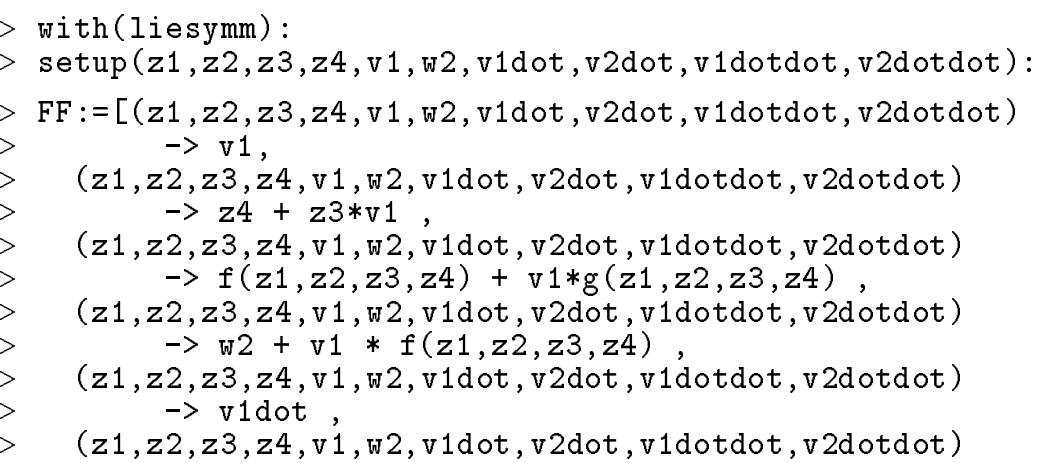

ESAIM: COCV, JUNE 1997, VOL. 2, PP. 151-230 


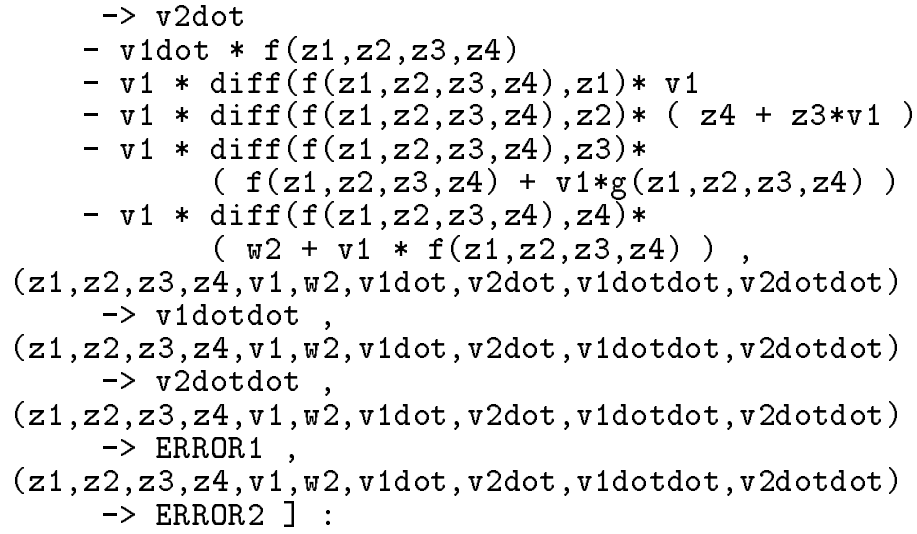

DOT := proc(forme) value(Lie(forme,FF)) end :

Here, we have loaded the package "liesymm" that we will use to manipulate differential forms, declared that the coordinates are $\left(z_{1}, z_{2}, z_{3}, z_{4}, v_{1}, w_{2}\right.$, $\left.\dot{v}_{1}, \dot{v}_{2}, \ddot{v}_{1}, \ddot{v}_{2}\right)$, defined the system, and finally the procedure "DOT", which is the time-derivative along the dynamics of the system, i.e. the Lie derivative along the vector field $F$ from equation (2.5), this vector field is truncated here, but as explained above it will not be applied to objects that involve other variables than $x_{1}, x_{2}, x_{3}, x_{4}, u_{1}, u_{2}, \dot{u}_{1}$ and $\dot{u}_{2}$. This Lie derivative on functions as well as on forms of any degree.

Let us just check that DOT is really what we think, by applying it to the base variable functions:

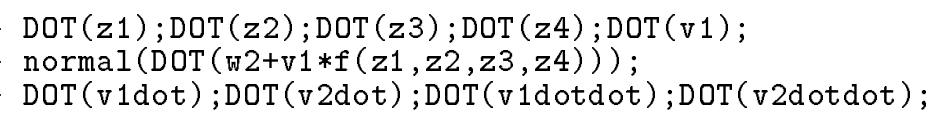

Note that the "ERROR" signs will never appear in the calculations since 
we will compute the time-derivative only of functions which do not depend on $\ddot{v}_{1}$ and $\ddot{v}_{2}$, see the explanations before $(7.101)$.

We now define the forms $\omega_{1}$ and $\omega_{2}$, and compute $\dot{\omega}_{1}, \dot{\omega}_{2}$ and $\ddot{\omega}_{2}$ (denoted "omega1d", "omega2d" and "omega2dd"):

$$
\begin{aligned}
& >\text { omega1 }:=\mathrm{d}(\mathrm{z} 2)-\mathrm{z} 3 * \mathrm{~d}(\mathrm{z} 1) ; \\
& \omega 1:=\mathrm{d}(z 2)-z 3 \mathrm{~d}(z 1) \\
& >\text { omega2 := wcollect(value( } \\
& d(z 3)-g(z 1, z 2, z 3, z 4) * d(z 1) \\
& -(\operatorname{diff}(f(z 1, z 2, z 3, z 4), z 4)+v 1 * \operatorname{diff}(g(z 1, z 2, z 3, z 4), z 4)) \\
& \text { * omega1)) ; } \\
& \omega 2:=(-\mathrm{g}(z 1, z 2, z 3, z 4) \\
& \left.+\left(\left(\frac{\partial}{\partial z / 4} \mathrm{f}\left(z 1, z 2, z 3, z_{4}\right)\right)+v 1\left(\frac{\partial}{\partial z_{4}} \mathrm{~g}\left(z 1, z 2, z 3, z_{4}\right)\right)\right) z 3\right) \mathrm{d}(z 1) \\
& +\left(-\left(\frac{\partial}{\partial z_{4}^{4}} \mathrm{f}\left(z 1, z^{2}, z_{3}, z_{4}\right)\right)-v 1\left(\frac{\partial}{\partial z_{4}} \mathrm{~g}\left(z 1, z^{2}, z_{3}, z_{4}\right)\right)\right) \mathrm{d}\left(z^{2}\right) \\
& +\mathrm{d}(z 3)
\end{aligned}
$$

$>$ omega1d $:=$ DOT $($ omega 1$):$ omega2d $:=$ DOT $($ omega 2$):$ omega2dd $:=$ $\rightarrow$ DOT (omega2d):

From proposition 2.11, the rank condition (4.5) implies that the form of degree $3 \omega_{1} \wedge \omega_{2} \wedge \dot{\omega}_{2}$ does not vanish. We compute it, see that it is of the form $D_{1} \mathrm{~d} z_{1} \wedge \mathrm{d} z_{2} \wedge \mathrm{d} z_{3}$ where the quantity $D_{1}$ is the one from (4.15). We shall use a lot the fact that this $D_{1}$ does not vanish.

$>$ D1 := getcoeff(factor( omega1\&`omega2\&`omega2d ));

$$
\begin{aligned}
& D 1:=\left(\frac{\partial}{\partial z 1} \mathrm{f}(z 1, z 2, z 3, z 4)\right)+z 3\left(\frac{\partial}{\partial z 2} \mathrm{f}\left(z 1, z 2, z 3, z_{4}\right)\right) \\
& +\mathrm{g}(z 1, z 2, z 3, z 4)\left(\frac{\partial}{\partial z 3} \mathrm{f}(z 1, z 2, z 3, z 4)\right) \\
& -\left(\frac{\partial}{\partial z_{4}} \mathrm{~g}\left(z 1, z 2, z_{3}, z_{4}\right)\right) w_{2} \\
& -\left(\frac{\partial}{\partial z 3} \mathrm{~g}\left(z 1, z 2, z 3, z_{4}\right)\right) \mathrm{f}\left(z 1, z 2, z 3, z_{4}\right) \\
& +\left(\frac{\partial}{\partial z 4} \mathrm{f}(z 1, z 2, z 3, z 4)\right) \mathrm{f}(z 1, z 2, z 3, z 4) \\
& -\left(\frac{\partial}{\partial z^{2}} \mathrm{~g}\left(z 1, z 2, z 3, z_{4}\right)\right) z_{4}^{4}
\end{aligned}
$$

This implements (7.102)-(7.103) and computes $\Omega_{1}, \Omega_{3}$ and $\Omega_{3}$ according to (7.55), $\Omega_{2}$ being the intermediary form defined in (7.68)-(7.70).

Note that the form $\Omega_{3}$ that we use is not exactly the one in (7.55), it is divided by $b$; this does not affect the relations (7.56)(7.57).

$>\mathrm{b}:=1 / \operatorname{beta}(\mathrm{z} 1, \mathrm{z} 2, \mathrm{z3}, \mathrm{z4}, \mathrm{v} 1$, w2,v1dot, v2dot);

$>$ alpha := b * rho(z1,z2,z3,z4,v1,w2,v1dot, v2dot);

Esaim: Cocv, June 1997, Vol. 2, Pf. 151-230 


$$
\begin{aligned}
& b:=\frac{1}{\beta\left(z 1, z 2, z 3, z 4, v 1, w_{2}, v 1 d o t, v_{2 d o t}\right)} \\
& \alpha:=\frac{\rho\left(z 1, z 2, z 3, z 4, v 1, w^{2}, v 1 d o t, v 2 d o t\right)}{\beta\left(z 1, z 2, z 3, z_{4}, v 1, w_{2}, v 1 d o t, v 2 d o t\right)}
\end{aligned}
$$

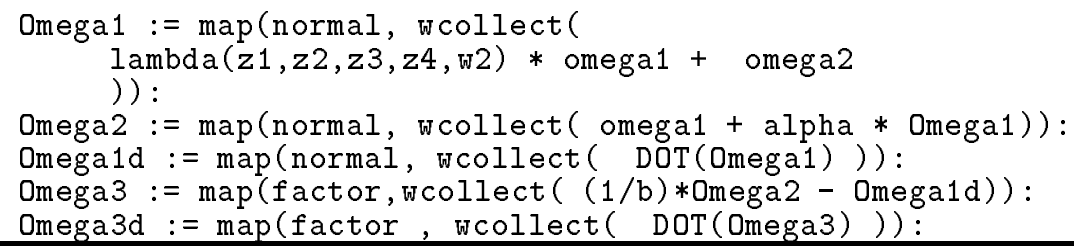

We shall first compute $\mathrm{d} \Omega_{1}$ modulo $\left\{\Omega_{1}, \Omega_{3}, \dot{\Omega}_{3}\right\}$. In order to compute modulo

$\left\{\Omega_{1}, \Omega_{3}, \dot{\Omega}_{3}\right\}$, we simply substitute $\mathrm{d} z_{3}, \mathrm{~d} z_{4}$ and $\mathrm{d} w_{2}$ with the linear combinations - respectively called valdz 3 , valdz 4 and valdw2 below- of $\mathrm{d} z_{1}, \mathrm{~d} z_{2}$, $\mathrm{d} v_{1}$ which is equal to each of them $\bmod u l o\left\{\Omega_{1}, \Omega_{3}, \dot{\Omega}_{3}\right\}$.

$>\operatorname{map}($ getform, $[$ op (Omega1)]);

$>\operatorname{coeff}($ Omega1 , d $(z 3))$;

$$
[\mathrm{d}(z 1), \mathrm{d}(z 2), \mathrm{d}(z 3)]
$$

$>$ valdz3 := map(factor, wcollect(solve( Omega1=0, d(z3)) )):

$>\operatorname{map}($ getform, $[o p(\operatorname{valdz} 3)])$;

$$
[\mathrm{d}(z 1), \mathrm{d}(z \mathscr{2})]
$$

$>\operatorname{map}($ getform, $[o p($ Omega3)]);

$>$ coeff( Omega3, d(z4));

$$
\begin{gathered}
{\left[\mathrm{d}(z 1), \mathrm{d}\left(z^{2}\right), \mathrm{d}\left(z_{3}\right), \mathrm{d}\left(z_{4}\right)\right]} \\
-\lambda\left(z 1, z_{2}, z_{3}, z_{4}, w_{2}\right)
\end{gathered}
$$

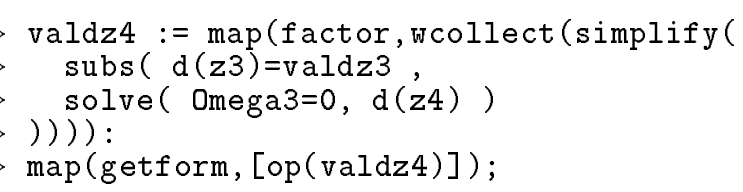

$$
[\mathrm{d}(z 1), \mathrm{d}(z 2)]
$$

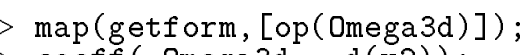

$$
\begin{gathered}
{\left[\mathrm{d}(z 1), \mathrm{d}(z 2), \mathrm{d}(z 3), \mathrm{d}(z 4), \mathrm{d}(v 1), \mathrm{d}\left(w_{2}\right)\right]} \\
-\lambda(z 1, z 2, z 3, z 4, w 2)
\end{gathered}
$$




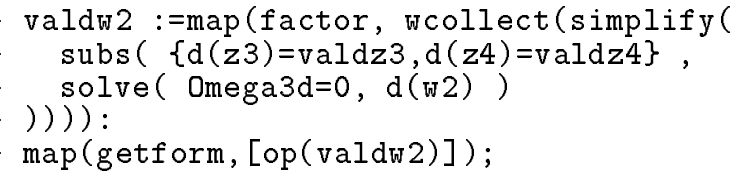

In other terms, $\mathrm{d} \Omega_{1} \equiv C_{1} \mathrm{~d} z_{1} \wedge \mathrm{d} z_{2}+C_{2} \mathrm{~d} z_{2} \wedge \mathrm{d} v_{1}+C_{3} \mathrm{~d} z_{1} \wedge \mathrm{d} v_{1}$ modulo $\left\{\Omega_{1}, \Omega_{3}, \dot{\Omega}_{3}\right\}$. Hence the functions $C_{1}, C_{2}$ and $C_{3}$ must be identically zero.

Let us first examine the coefficient of $\mathrm{d} z_{2} \wedge \mathrm{d} v_{1}$. It turns out that $C_{2}=0$ allows one to express $\frac{\partial \lambda}{\partial w_{2}}$ as a function of $f, g, \lambda$. It is the expression "LALA" below.

$$
\begin{aligned}
& >\operatorname{collect}\left(\text { coeff(d0mega1mod, } \&^{\wedge}(\mathrm{d}(\mathrm{z} 2), \mathrm{d}(\mathrm{v} 1))\right) \text {, } \\
& \operatorname{diff}(1 \operatorname{ambda}(z 1, z 2, z 3, z 4, \text { w2 }) \text {, w2) ); } \\
& -\left(-\left(\frac{\partial}{\partial z 4} \mathrm{f}(z 1, z 2, z 3, z 4)\right) \mathrm{f}(z 1, z 2, z 3, z 4)\right. \\
& -z 3\left(\frac{\partial}{\partial z 2} \mathrm{f}(z 1, z 2, z 3, z 4)\right) \\
& -\left(\frac{\partial}{\partial z 1} \mathrm{f}\left(z 1, z 2, z 3, z_{4}\right)\right)+\left(\frac{\partial}{\partial z_{4}} \mathrm{~g}\left(z 1, z 2, z 3, z_{4}\right)\right) w_{2} \\
& +\left(\frac{\partial}{\partial z 3} \mathrm{~g}\left(z 1, z 2, z 3, z_{4}\right)\right) \mathrm{f}\left(z 1, z 2, z 3, z_{4}\right) \\
& +\left(\frac{\partial}{\partial z^{2}} \mathrm{~g}\left(z 1, z 2, z 3, z_{4}\right)\right) z_{4} \\
& \left.-\mathrm{g}(z 1, z 2, z 3, z 4)\left(\frac{\partial}{\partial z 3} \mathrm{f}(z 1, z 2, z 3, z 4)\right)\right) \\
& \left(\frac{\partial}{\partial w^{2}} \lambda\left(z 1, z 2, z 3, z_{4}, w 2\right)\right) /\left(\lambda\left(z 1, z 2, z 3, z_{4}, w_{2}\right)\right) \\
& +\left(\frac{\partial}{\partial z / 4} \mathrm{~g}\left(z 1, z 2, z 3, z_{4}\right)\right)
\end{aligned}
$$

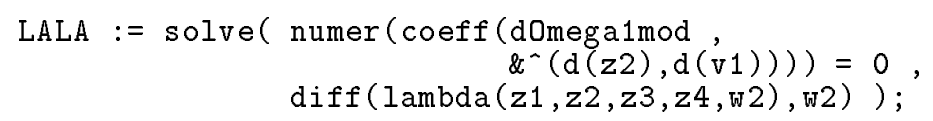




$$
\begin{aligned}
& L A L A:=-\left(\frac{\partial}{\partial z_{4}} \mathrm{~g}\left(z 1, z 2, z 3, z_{4}\right)\right) \lambda\left(z 1, z 2, z_{3}, z_{4}, w 2\right) /( \\
& \left(\frac{\partial}{\partial z 1} \mathrm{f}(z 1, z 2, z 3, z 4)\right)+z 3\left(\frac{\partial}{\partial z^{2}} \mathrm{f}(z 1, z 2, z 3, z 4)\right) \\
& +\mathrm{g}\left(z 1, z 2, z 3, z_{4}\right)\left(\frac{\partial}{\partial z 3} \mathrm{f}\left(z 1, z 2, z 3, z_{4}\right)\right) \\
& -\left(\frac{\partial}{\partial z_{4}} \mathrm{~g}\left(z 1, z 2, z_{3}, z_{4}\right)\right) w_{2} \\
& -\left(\frac{\partial}{\partial z 3} \mathrm{~g}\left(z 1, z 2, z 3, z_{4}\right)\right) \mathrm{f}\left(z 1, z 2, z 3, z_{4}\right) \\
& +\left(\frac{\partial}{\partial z 4} \mathrm{f}(z 1, z 2, z 3, z 4)\right) \mathrm{f}(z 1, z 2, z 3, z 4) \\
& \left.-\left(\frac{\partial}{\partial z^{2}} \mathrm{~g}\left(z 1, z 2, z 3, z_{4}\right)\right) z_{4}\right)
\end{aligned}
$$

The general solution of the linear PDE $\frac{\partial \lambda}{\partial w_{2}}=L A L A$ is LALALAsymb below, where the function " $c c$ " has to be equal to "coco" also given below, and $\lambda_{0}$ is a free function of four variables.

$>$ LALALAsymb $:=\operatorname{lambda0}(z 1, z 2, z 3, z 4) *(w 2+c c(z 1, z 2, z 3, z 4))$;

$L A L A L A \operatorname{symb}:=\lambda 0\left(z 1, z \mathscr{2}, z 3, z_{4}\right)\left(w_{2}+\operatorname{cc}\left(z 1, z 2, z 3, z_{4}\right)\right)$

$$
\begin{aligned}
& >\operatorname{coco}:=\operatorname{factor}((\operatorname{lambda}(z 1, z 2, z 3, z 4, \text { w2 }) / \text { LALA })-\text { w2 }) \text {; } \\
& \operatorname{coco}:=\left(\left(\frac{\partial}{\partial z^{2}} \mathrm{~g}\left(z 1, z 2, z 3, z_{4}\right)\right) z_{4}-z 3\left(\frac{\partial}{\partial z 2} \mathrm{f}\left(z 1, z 2, z 3, z_{4}\right)\right)\right. \\
& -\left(\frac{\partial}{\partial z 4} \mathrm{f}\left(z 1, z 2, z 3, z_{4}\right)\right) \mathrm{f}\left(z 1, z 2, z 3, z_{4}\right)-\left(\frac{\partial}{\partial z 1} \mathrm{f}\left(z 1, z 2, z 3, z_{4}\right)\right) \\
& -\mathrm{g}\left(z 1, z 2, z 3, z_{4}\right)\left(\frac{\partial}{\partial z 3} \mathrm{f}\left(z 1, z 2, z_{3}, z_{4}\right)\right) \\
& \left.+\left(\frac{\partial}{\partial z 3} \mathrm{~g}\left(z 1, z 2, z 3, z_{4}\right)\right) \mathrm{f}(z 1, z 2, z 3, z 4)\right) /( \\
& \left.\frac{\partial}{\partial z_{4}} \mathrm{~g}\left(z 1, z 2, z 3, z_{4}\right)\right)
\end{aligned}
$$

In the sequel, we shall substitute $\lambda$ with the expression LALALAsymb rather than with the expression 


$$
\begin{aligned}
& \lambda 0(z 1, z 2, z 3, z 4)\left(-\left(\frac{\partial}{\partial z_{4}} \mathrm{f}(z 1, z 2, z 3, z 4)\right) \mathrm{f}(z 1, z 2, z 3, z 4)\right. \\
& -z 3\left(\frac{\partial}{\partial z^{2}} \mathrm{f}\left(z 1, z 2, z 3, z_{4}\right)\right)-\left(\frac{\partial}{\partial z 1} \mathrm{f}\left(z 1, z^{2}, z_{3}, z_{4}\right)\right) \\
& +\left(\frac{\partial}{\partial z_{4}} \mathrm{~g}(z 1, z 2, z 3, z 4)\right) w_{2} \\
& +\left(\frac{\partial}{\partial z 3} \mathrm{~g}(z 1, z 2, z 3, z 4)\right) \mathrm{f}(z 1, z 2, z 3, z 4) \\
& +\left(\frac{\partial}{\partial z^{2}} \mathrm{~g}\left(z 1, z^{2}, z_{3}, z_{4}\right)\right) z_{4} \\
& \left.-\mathrm{g}\left(z 1, z_{2}, z_{3}, z_{4}\right)\left(\frac{\partial}{\partial z_{3}} \mathrm{f}\left(z 1, z^{2}, z_{3}, z_{4}\right)\right)\right) /( \\
& \left.\frac{\partial}{\partial z_{4}} \mathrm{~g}\left(z 1, z 2, z 3, z_{4}\right)\right)
\end{aligned}
$$

where $\operatorname{cc}(\mathrm{z} 1, \mathrm{z} 2, \mathrm{z} 3, \mathrm{z} 4)$ is replaced by its value, because it makes the expressions shorter (without this trick, 100MegaBytes were not enough to run the Maple session).

Actually, we will rather substitute $\frac{\partial f}{\partial z_{1}}$ with its expression below, as a function of other partial derivatives and of the function $c c$ than doing the contrary:

$$
\begin{aligned}
& >\text { valdfdz1:= factor }(\text { solve }(\operatorname{coco}=c c(z 1, z 2, z 3, z 4) \text {, } \\
& \operatorname{diff}(f(z 1, z 2, z 3, z 4), z 1))) \text {; } \\
& \text { valdfd } z 1:=\left(\frac{\partial}{\partial z^{2}} \mathrm{~g}\left(z 1, z^{2}, z 3, z_{4}\right)\right) z_{4}-z_{3}\left(\frac{\partial}{\partial z^{2}} \mathrm{f}\left(z 1, z 2, z 3, z_{4}\right)\right) \\
& -\left(\frac{\partial}{\partial z 4} \mathrm{f}\left(z 1, z 2, z 3, z_{4}\right)\right) \mathrm{f}\left(z 1, z 2, z 3, z_{4}\right) \\
& -\mathrm{g}(z 1, z 2, z 3, z 4)\left(\frac{\partial}{\partial z 3} \mathrm{f}(z 1, z 2, z 3, z 4)\right) \\
& +\left(\frac{\partial}{\partial z 3} \mathrm{~g}(z 1, z 2, z 3, z 4)\right) \mathrm{f}\left(z 1, z 2, z 3, z_{4}\right) \\
& -\operatorname{cc}\left(z 1, z^{2}, z 3, z_{4}\right)\left(\frac{\partial}{\partial z_{4}} \mathrm{~g}\left(z 1, z^{2}, z 3, z_{4}\right)\right)
\end{aligned}
$$

The routine "subslambda" replaces $\lambda$ with its expression LALALAsymb in an expression.

The routine "subsdfdz1" replaces $\frac{\partial f}{\partial z_{1}}$ with its expression subsdfdz1 in an expression, it is slightly more complicated to take care of substitutions in higher order partial derivatives of $f$

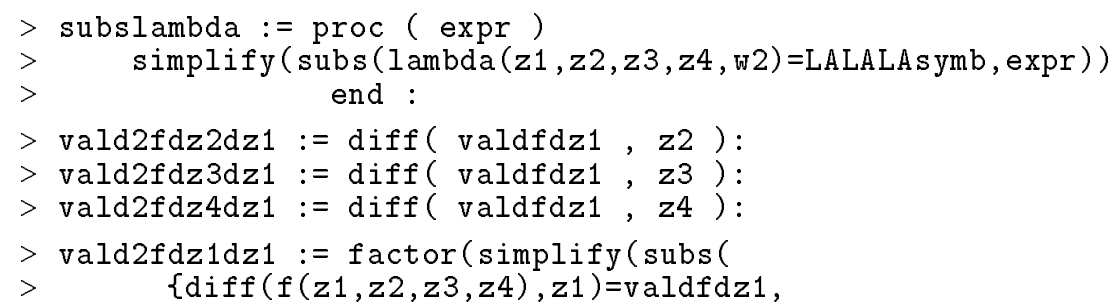

Esaim: CoCv, June 1997, Vol. 2, PP. 151-230 


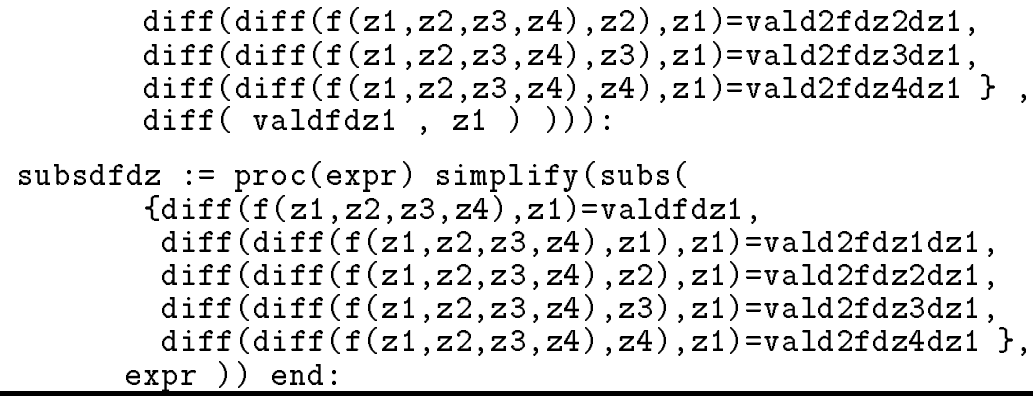

Let us see the coefficient of $\mathrm{d} z_{1} \wedge \mathrm{d} z_{2}$ now. It turns out that it is affine with respect to the function $\beta$ with the coefficient below in front of $\beta$. Hence, $C_{1}=0$ may be solved explicitly for $\beta$, the expression for $\beta$ is called "valbeta".

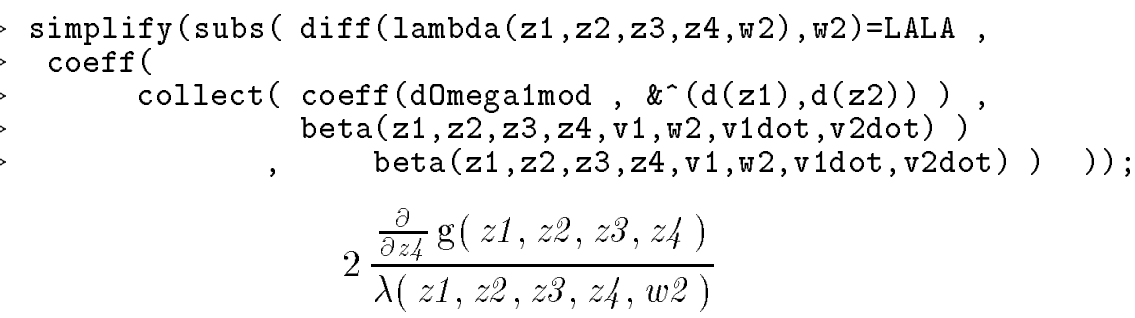

Let us replace $\lambda$ by its value, and $\frac{\partial f}{\partial z_{1}}$ by the expression subsdfdz1 in valbeta and call the new expression valbetaS:

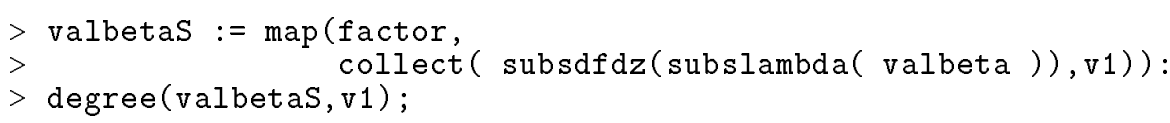

3

The expression valbetaS is polynomial of degree 3 with respect to $v_{1}$.

From (7.56), we got an expression for $\frac{\partial \lambda}{\partial w_{2}}$ (LALA, from which we derived an expression for $\lambda$ summed up in the substitution routines "subslambda" and "subsdfdz1") and an expression for $\beta$ (valbetaS). Let us check that it is all we can get, i.e. these substitution make equation (7.56) trivially true:

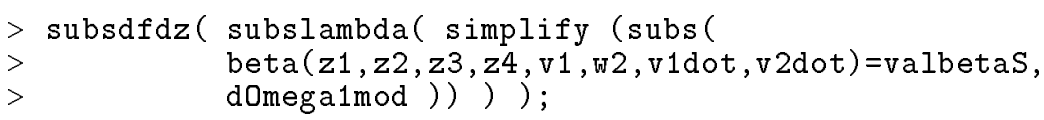

We now turn to (7.57), i.e. to $\mathrm{d} \Omega_{3}$.

$>$ domega3 $:=$ factor ( value (d(Omega3)) ) :

The routine "modOm 3 " below computes the expression of a form modulo Omega $a_{3}$ by substituting $\mathrm{d} z_{4}$ with "valdz4", the linear combination of $\mathrm{d} z_{1}$, $\mathrm{d} z_{2}$ and $\mathrm{d} z_{3}$ which is equivalent to $\mathrm{d} z_{4} \operatorname{modulo} \Omega_{3}$ :

$>\operatorname{map}($ getform, $[o p($ wcollect(Omega3))]) ; coeff( Omega3, d(z4));

$$
\begin{gathered}
{\left[\mathrm{d}(z 1), \mathrm{d}\left(z^{2}\right), \mathrm{d}\left(z^{3}\right), \mathrm{d}\left(z_{4}\right)\right]} \\
-\lambda\left(z 1, z^{2}, z_{3}, z_{4}, w_{2}\right)
\end{gathered}
$$




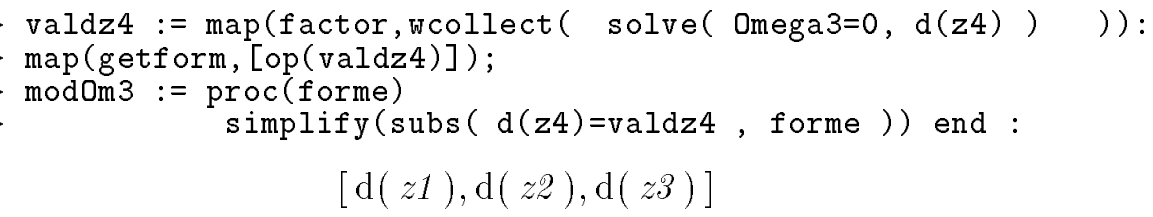

Let us now compute $d \Omega_{3}$ modulo $\Omega_{3}$. In the expression "dOmega3mod" below, not only have we performed the above substitution, but we have also removed all the terms containing $\mathrm{d} \dot{v}_{1}$ or $\mathrm{d} \dot{v}_{2}$, which will be useless...

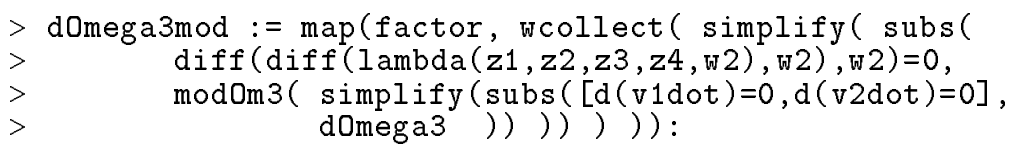

Let us check that the forms that appear in dOmega 3 and dOmega 3 mod are these we expect:

$$
\begin{aligned}
& >\operatorname{map}(\text { getform, }[o p(\text { wcollect (d0mega3)) }]) \text {; } \\
& >\operatorname{map}(\text { getform, [op(d0mega3mod)]) ; } \\
& {\left[\mathrm{d}(z 1) \&^{\wedge} \mathrm{d}\left(z_{4}\right), \mathrm{d}(z 1) \&^{\wedge} \mathrm{d}\left(w^{2}\right), \mathrm{d}\left(z^{2}\right) \&^{\wedge} \mathrm{d}\left(w^{2}\right), \mathrm{d}(z 1) \&^{\wedge} \mathrm{d}(v 1),\right.} \\
& \mathrm{d}\left(z_{\mathscr{L}}\right) \&^{\wedge} \mathrm{d}\left(z_{4}\right), \mathrm{d}\left(z_{\mathscr{L}}\right) \&^{\wedge} \mathrm{d}(v 1 d o t), \mathrm{d}\left(z^{2}\right) \&^{\wedge} \mathrm{d}\left(v_{2} d o t\right), \\
& \mathrm{d}\left(z^{2}\right) \&^{\wedge} \mathrm{d}(v 1), \mathrm{d}\left(z_{4}\right) \&^{\wedge} \mathrm{d}\left(w_{2}\right), \mathrm{d}(z 1) \&^{\wedge} \mathrm{d}(z 2), \\
& \mathrm{d}(z 2) \&^{\wedge} \mathrm{d}(z 3), \mathrm{d}(z 1) \&^{\wedge} \mathrm{d}(z 3), \mathrm{d}(z 3) \&^{\wedge} \mathrm{d}(z 4), \\
& \mathrm{d}(z 3) \&^{\wedge} \mathrm{d}(v 1), \mathrm{d}(z 3) \&^{\wedge} \mathrm{d}\left(\text { w2) }, \mathrm{d}(z 3) \&^{\wedge} \mathrm{d}(v 1 d o t),\right. \\
& \left.\mathrm{d}(z 3) \&^{\wedge} \mathrm{d}(v 2 d o t), \mathrm{d}(z 1) \&^{\wedge} \mathrm{d}(v 1 d o t), \mathrm{d}(z 1) \&^{\wedge} \mathrm{d}(v 2 d o t)\right] \\
& {\left[\mathrm{d}(z 1) \&^{\wedge} \mathrm{d}\left(w_{2}\right), \mathrm{d}\left(z^{2}\right) \&^{\wedge} \mathrm{d}\left(w_{2}\right), \mathrm{d}(z 1) \&^{\wedge} \mathrm{d}(v 1), \mathrm{d}\left(z^{2}\right) \&^{\wedge} \mathrm{d}(v 1),\right.} \\
& \mathrm{d}(z 1) \&^{\wedge} \mathrm{d}(z 2), \mathrm{d}(z 2) \&^{\wedge} \mathrm{d}(z 3), \mathrm{d}(z 1) \&^{\wedge} \mathrm{d}(z 3), \\
& \mathrm{d}(z 3) \&^{\wedge} \mathrm{d}(v 1), \mathrm{d}(z 3) \&^{\wedge} \mathrm{d}(\text { w2) }]
\end{aligned}
$$

We now compute $\Omega_{1} \wedge \dot{\Omega}_{3}$ modulo $\Omega_{3}$, it is the expression called "Omega33dmod".

$>\operatorname{map}($ getform, $[$ op (Omega3d)]);

$$
\left[\mathrm{d}(z 1), \mathrm{d}\left(z^{2}\right), \mathrm{d}(z 3), \mathrm{d}\left(z_{4}\right), \mathrm{d}(v 1), \mathrm{d}\left(w_{2}\right)\right]
$$

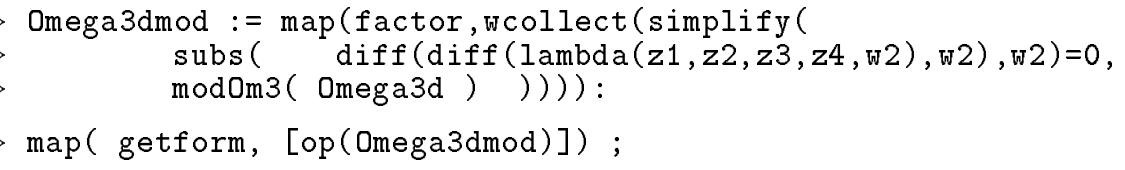

Omega33dmod := map (factor, wcollect(Omega1 \&^Omega3dmod)):

The coefficient of $\mathrm{d} z_{3}$ is rather simple in "dOmega3mod" and "Omega33dmod":

$>\operatorname{cc1}:=-\operatorname{coeff}\left(\right.$ Omega33dmod,$\left.\&^{-}(\mathrm{d}(\mathrm{z} 3), \mathrm{d}(\mathrm{w} 2))\right) ;$
$>\operatorname{cc} 2:=\operatorname{coeff}\left(\mathrm{dOmega3mod}, \&^{\wedge}(\mathrm{d}(\mathrm{z} 3), \mathrm{d}(\mathrm{w} 2))\right) ;$

$$
c c 1:=\lambda\left(z 1, z^{2}, z^{3}, z 4, w^{2}\right)
$$




$$
\begin{aligned}
& \operatorname{cc\mathscr {D}}:=\left(\% 1 v 1\left(\frac{\partial}{\partial z 4} \mathrm{f}(z 1, z 2, z 3, z 4)\right)\right. \\
& +\% 1 \rho\left(z 1, z 2, z 3, z_{4}, v 1, w_{2}, v 1 d o t, v 2 d o t\right) \\
& -\% 1\left(\frac{\partial}{\partial z 3} \mathrm{f}\left(z 1, z^{2}, z_{3}, z_{4}\right)\right)-\% 1 v 1\left(\frac{\partial}{\partial z 3} \mathrm{~g}\left(z 1, z 2, z 3, z_{4}\right)\right) \\
& +\% 1 v 1^{2}\left(\frac{\partial}{\partial z 4} \mathrm{~g}(z 1, z 2, z 3, z 4)\right)- \\
& \left(\frac{\partial}{\partial w^{2}} \rho\left(z 1, z^{2}, z_{3}, z_{4}, v 1, w_{2}, v 1 d o t, v 2 d o t\right)\right) \lambda\left(z 1, z 2, z 3, z 4, w_{2}\right) \\
& ) /(\lambda(z 1, z 2, z 3, z 4, w 2)) \\
& \% 1:=\frac{\partial}{\partial w^{2}} \lambda\left(z 1, z 2, z^{3}, z_{4}, w_{2}\right) \\
& >\text { factor }\left(\mathrm { cc } 1 * \operatorname { c o e f f } \left(\text { d0mega3mod, } \mathbb{L}^{-}(\mathrm{d}(\mathrm{z} 3), \mathrm{d}(\text { w2) }))\right.\right. \\
& \left.>\quad+\mathrm{cc} 2 * \operatorname{coeff}\left(\text { Omega33dmod, } \mathrm{E}^{-}(\mathrm{d}(\mathrm{z} 3), \mathrm{d}(\mathrm{w} 2))\right)\right) \text {; }
\end{aligned}
$$

0

Equation (7.57) implies cc1dOmega 3 unmod $-c c 2$ Omega $33 d$ mod $=0$. It turns out that the coefficient of $\mathrm{d} z_{2} \wedge \mathrm{d} w_{2}$ allows one to solve for $\rho$ :

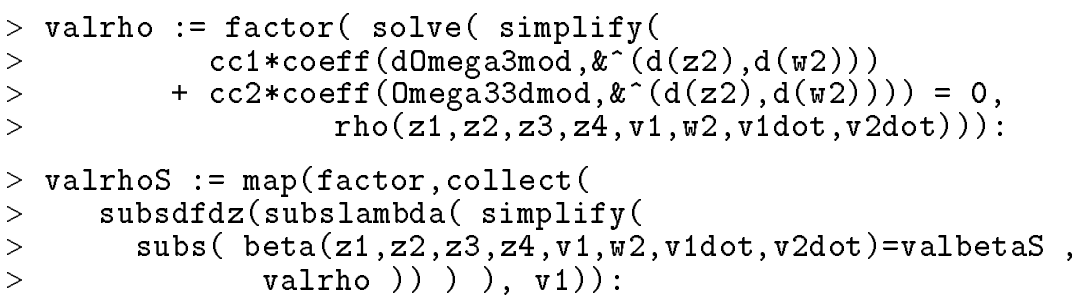

valrhos is an expression of valrho where $\beta$ and $\lambda$ are substituted for the values computed above. It is shorter than valrho:

$>[$ nops $(\operatorname{expand}($ numer $(\operatorname{valrho})))$, nops $(\operatorname{expand}($ numer $(\operatorname{valrhoS})))]$;

$$
[56,23]
$$

Let us now compute the coefficient of $\mathrm{d} z_{2} \wedge \mathrm{d} w_{2}$, and call it EE:

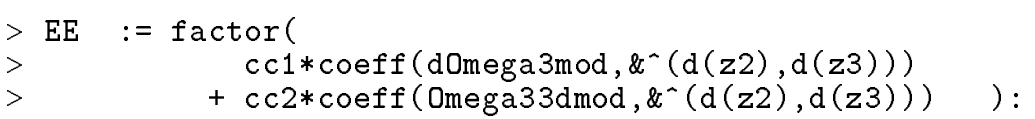

It turns out that it is a rather large expression:

$>\operatorname{nops}(\operatorname{numer}(\mathrm{EE})) ; \operatorname{denom}(E E)$;

7510

$$
\lambda(z 1, z \mathscr{2}, z 3, z 4, w 2)^{2}
$$

This expression is large enough that if we simply substitute $\beta$ and rho with valrhos and valbetaS, it takes more that $100 \mathrm{MBytes}$ to compute the result.

To round this problem, we shall take advantage of the fact that valbetaS, valrhoS and $\mathrm{EE}$ are polynomial with respect to $v_{1}$, and use the expressions 
"rho77" and "beta77" which are polynomials of the right degree in the indeterminate $v_{1}$, with coefficients some generic functions of $(z 1, z 2, z 3, z 4, w 2) \ldots$ Of course, when these coefficients are substituted for the right functions, "rho77" and "beta77" have the same values as "valrhoS" and "valbetaS"

$>$ degree(valbetaS,v1); degree(valrhoS,v1);

$>$ BB3 $:=\operatorname{coeff}($ valbetaS, 1,3$)$
$>$ BB2 $:=\operatorname{coeff}($ valbetaS, 1,2$):$
$>$ BB1 $:=\operatorname{coeff}($ valbetaS, v1 1$):$
$>$ BBO $:=$ coeff $($ valbetaS, v1, 0$):$

$$
B B 3:=-\left(\frac{\partial}{\partial z 4} \mathrm{~g}(z 1, z 2, z 3, z 4)\right)^{2}
$$

$>$ RR2 := coeff( valrhoS, v1, 2);

$>$ RR1 $:=$ coeff ( valrhos, v1, 1$)$;

$>$ RRO $:=\operatorname{coeff}($ valrhos, $\mathrm{v} 1,0)$ :

$$
R R 2:=-\left(\frac{\partial}{\partial z_{4}} \mathrm{~g}\left(z 1, z^{2}, z_{3}, z_{4}\right)\right)
$$

$R R 1:=2 \lambda 0(z 1, z 2, z 3, z 4) \operatorname{cc}(z 1, z 2, z 3, z 4)+2 \lambda 0(z 1, z 2, z 3, z 4) w 2$

$$
\begin{aligned}
& -\left(\frac{\partial}{\partial z 4} \mathrm{f}\left(z 1, z 2, z 3, z_{4}\right)\right)+\left(\frac{\partial}{\partial z 3} \mathrm{~g}(z 1, z 2, z 3, z 4)\right) \\
& >\text { rho77:= RR2* v1^2+RR1*v1 + R0 (z1,z2,z3,z4,w2): } \\
& \text { beta77 := BB3 *v1^3+B2(z1,z2,z3,z4,w2)*v1^2 } \\
& +\mathrm{B} 1(\mathrm{z} 1, \mathrm{z} 2, \mathrm{z} 3, \mathrm{z} 4, \mathrm{w} 2) * \mathrm{v} 1+\mathrm{BO}(\mathrm{z} 1, \mathrm{z} 2, \mathrm{z3}, \mathrm{z} 4, \mathrm{w} 2):
\end{aligned}
$$

Let us check that when performing the correct substitutions, beta77 = valbetaS and rho77 $=$ valrhoS

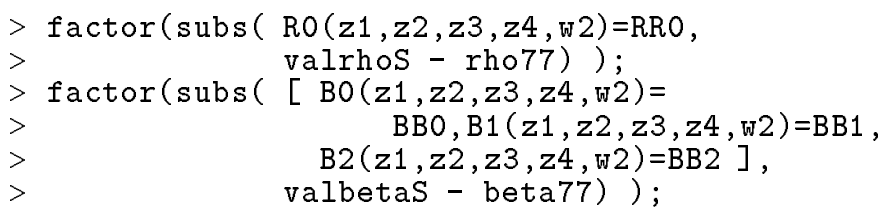

0

0

Now we substitute in the expression EE :

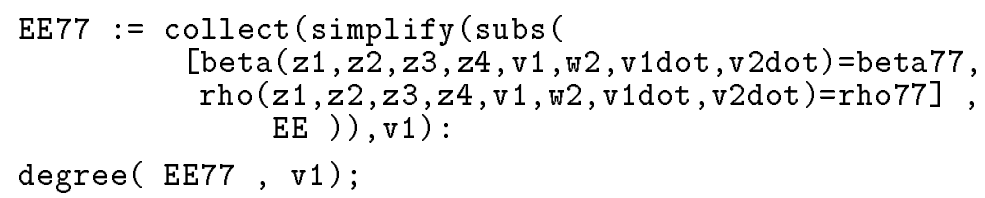

5

The leading coefficient turns out to be zero when substituting $\lambda$ :

$>$ factor ( subslambda( coeff(EE77, v1, 5 ) )); 
And the coefficient of degree 4 is

$$
\begin{aligned}
& >\text { factor ( subsdfdz(subslambdal simplify ( } \\
& \operatorname{subs}([\mathrm{RO}(z 1, z 2, z 3, z 4, \mathrm{w} 2)=\mathrm{RR} 0 \\
& \mathrm{B} 2(z 1, z 2, z 3, z 4, \text { w2 })=\mathrm{BB} 2] \\
& \operatorname{coeff}(\operatorname{EE77}, \mathrm{v1}, 4) \text { )) ) ) ); } \\
& \frac{1}{4}\left(\frac{\partial}{\partial z_{4}} \mathrm{~g}\left(z 1, z^{2}, z_{3}, z_{4}\right)\right)\left(w_{2}+\operatorname{cc}\left(z 1, z^{2}, z 3, z_{4}\right)\right)^{2} \lambda 0\left(z 1, z^{2}, z_{3}, z_{4}\right)^{3}
\end{aligned}
$$

It has to be zero, hence $\lambda$ is identically zero.

STEP 4. If $\alpha, \lambda$ and $b$ satisfy (7.56) with $\lambda$ identically zero, then $\delta_{2,1}^{1}$ cannot vanish and $b$ must be given by

$$
b=\frac{2 \gamma}{\delta_{2,1}^{1}} .
$$

This may easily be proved without the help of the program Maple.

Since $\lambda=0$, we have $\Omega_{1}=\omega_{2}, \Omega_{3}=\omega_{1}-b \dot{\omega}_{2}+\alpha \omega_{2}$, but from lemma 7.6, $\mathrm{d} \Omega_{1}$ satisfies $(7.45)$, i.e.

$$
\mathrm{d} \omega_{2} \equiv 0 \quad \operatorname{modulo}\left\{\omega_{2}, \Omega_{3}, \dot{\Omega}_{3}\right\} \text {. }
$$

From lemma 7.8 with $\Omega=\Omega_{3}, \lambda_{1}=1, \lambda_{2}=\alpha$ and $\lambda_{3}=-b$, the above relation implies that $b\left(b \delta_{2,1}^{1}-2 \gamma\right)$ is identically zero on $U$, but we assume here that $b$ does not vanish, hence $b \delta_{2,1}^{1}-2 \gamma$ must be identically zero, and therefore $\delta_{2,1}^{1}$ does not vanish (because $\gamma$ does not vanish), and $b$ is given by (7.104).

STEP 5. If $\alpha, \lambda$ and $b$ satisfy (7.56)-(7.57) with $\lambda$ identically zero and $b$ is given by $b=\frac{2 \gamma}{\delta_{2,1}^{1}}$, there is a unique possible value for $\alpha$, and $f$ and $g$ must be of the form (4.16)-(4.17).

This is done in the following Maple session which is the continuation of the previous one.

Session run with Maple $V$ Release 3 , continued

All the previous definitions remain valid, but we assign $\lambda$ to be identically zero:

$>$ lambda $:=\operatorname{proc}(z 1, z 2, z 3, z 4$, w2) 0 end :

First we need to compute $b$-i.e. $\beta$ - i.e. implement step 4 . To this end, we compute $d \Omega_{1} \wedge \Omega_{1} \wedge \Omega_{3} \wedge \dot{\Omega}_{3}$.

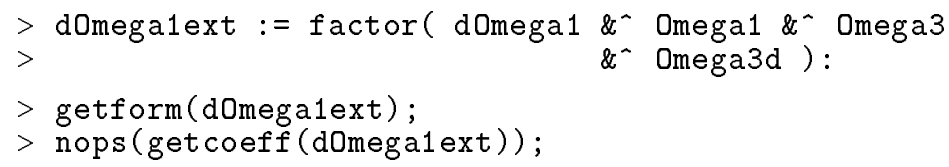

$$
\&^{\wedge}(\mathrm{d}(z 1), \mathrm{d}(z 2), \mathrm{d}(z 3), \mathrm{d}(z 4), \mathrm{d}(v 1))
$$

This is a monomial form of degree 5, whose coefficient must therefore be identically zero. We divide by $D_{1}$ which does not vanish :

$$
>\text { EEE1 := factor ( getcoeff(d0mega1ext) / (-D1) ): }
$$

This expression EEE1 is affine with respect to the function $\beta$, and its coefficient does not vanish :

$>\operatorname{coeff}(\mathrm{EEE1}, \operatorname{beta}(z 1, z 2, z 3, z 4, v 1, w 2, v 1 d o t, v 2 \operatorname{dot}))$; 


$$
-2\left(\frac{\partial}{\partial z 4} \mathrm{~g}(z 1, z 2, z 3, z 4)\right)
$$

One may therefore get $\beta$ from the equation EEE1 $=0$. Below, "valbeta" is the value of $\beta$ as the solution of EEE $1=0$. For convenience, we call $D_{2}$ the constant term in EEE1, so that $\beta$ will be equal to $D_{2} /\left(2 \frac{\partial g}{\partial z_{4}}\right)$ :

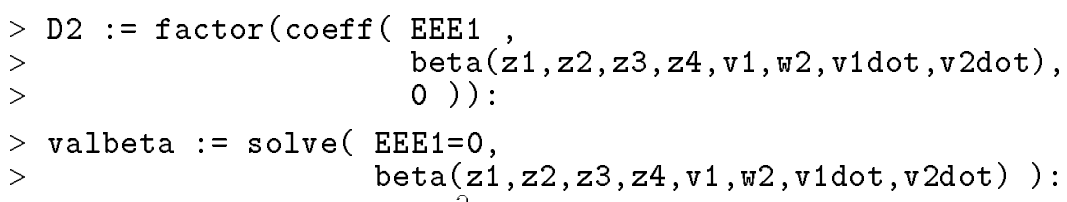

Let us check that $\beta=D_{2} /\left(2 \frac{\partial g}{\partial z_{4}}\right)$ :

$>\operatorname{normal}(\operatorname{valbeta}-\mathrm{D} 2 /(2 * \operatorname{diff}(\mathrm{g}(\mathrm{z1}, \mathrm{z2}, \mathrm{z3}, \mathrm{z4}), \mathrm{z4})))$;

Here we shall check that the only possible value for $b$-i.e. $1 /$ valbeta with valbeta computed above - is given by $\frac{2 \gamma}{\delta_{2,1}^{1}}$. Let us first compute $\gamma$ and $\delta_{2,1}^{1}$ and then check the equality. To compute $\gamma$ and $\delta_{2,1}^{1}$, we use the fact that, from (4.8),
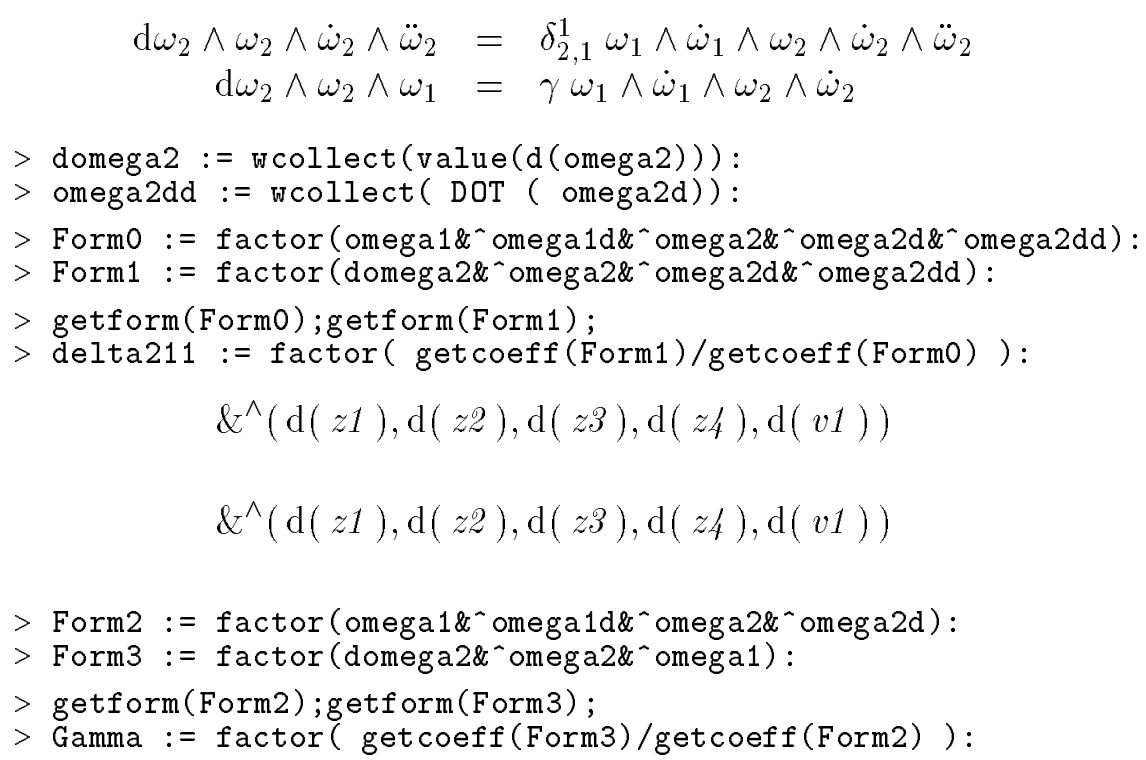

$$
\begin{aligned}
& \&^{\wedge}\left(\mathrm{d}(z 1), \mathrm{d}\left(z^{2}\right), \mathrm{d}(z 3), \mathrm{d}\left(z_{4}\right)\right) \\
& \&^{\wedge}\left(\mathrm{d}(z 1), \mathrm{d}\left(z^{2}\right), \mathrm{d}(z 3), \mathrm{d}\left(z_{4}\right)\right)
\end{aligned}
$$

$\delta_{2,1}^{1}$ is equal to $-D_{2} / D_{1}$ and $\gamma$ to $-\frac{\partial g}{\partial z_{4}} / D_{1}$

$>$ factor $(\operatorname{delta} 211 * \mathrm{D} 1 / \mathrm{D} 2)$;

$>$ factor (Gamma * D1) ;

$$
-\left(\frac{\partial}{\partial z_{4}} \mathrm{~g}(z 1, z 2, z 3, z 4)\right)
$$


Hence $\frac{2 \gamma}{\delta_{21}^{1}}=2 \frac{\partial g}{\partial z_{4}} / D_{2}$, which is equal to $1 /$ valbeta.

\footnotetext{
To compute $\rho$, we shall compute $\mathrm{d} \Omega_{3} \operatorname{modulo}\left\{\Omega_{1}, \Omega_{3}\right\}$, which is a fortiori zero from (7.57).

In order to compute modulo $\left\{\Omega_{1}, \Omega_{3}\right\}$, we simply substitute $\mathrm{d} z_{3}, \mathrm{~d} z_{2}$ with "valdz3" and "valdz2", the monomial forms in $\mathrm{d} z_{1}$ which is equal to each of them modulo $\left\{\Omega_{1}, \Omega_{3}\right\}$.
}

$>\operatorname{coeff}($ Omega1, d (z3));

$>\operatorname{vvaldz3}:=\operatorname{solve}($ Omega1=0, d(z3) );

$\operatorname{vvaldz3}:=\mathrm{d}(z 1) \mathrm{g}(z 1, z 2, z 3, z 4)-\mathrm{d}(z 1) z_{3}\left(\frac{\partial}{\partial z_{4}} \mathrm{f}\left(z 1, z 2, z_{3}, z_{4}\right)\right)$

$$
\begin{aligned}
& -\mathrm{d}(z 1) z_{3} v 1\left(\frac{\partial}{\partial z_{4}} \mathrm{~g}\left(z 1, z^{2}, z_{3}, z_{4}\right)\right) \\
& +\mathrm{d}\left(z_{2}\right)\left(\frac{\partial}{\partial z_{4}} \mathrm{f}\left(z 1, z^{2}, z_{3}, z_{4}\right)\right)+\mathrm{d}\left(z_{2}\right) v 1\left(\frac{\partial}{\partial z_{4}} \mathrm{~g}\left(z 1, z_{2}, z_{3}, z_{4}\right)\right)
\end{aligned}
$$

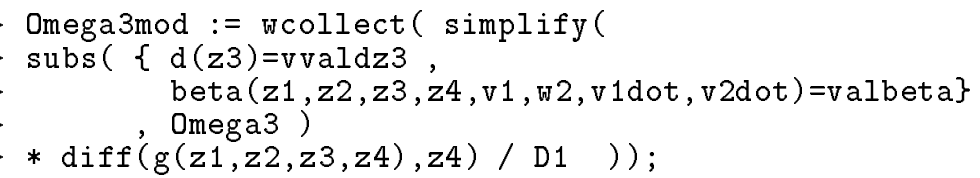

Omega3mod $:=\left(\frac{1}{2} z 3 v 1\left(\frac{\partial^{2}}{\partial z_{4}^{2}} \mathrm{~g}\left(z 1, z 2, z 3, z_{4}\right)\right)\right.$

$\left.-\left(\frac{\partial}{\partial z_{4}} \mathrm{~g}\left(z 1, z 2, z 3, z_{4}\right)\right)+\frac{1}{2} z 3\left(\frac{\partial^{2}}{\partial z_{4}^{2}} \mathrm{f}\left(z 1, z 2, z_{3}, z_{4}\right)\right)\right) \mathrm{d}(z 1)$

$+\left(-\frac{1}{2} v 1\left(\frac{\partial^{2}}{\partial z 4^{2}} \mathrm{~g}(z 1, z 2, z 3, z 4)\right)\right.$

$\left.-\frac{1}{2}\left(\frac{\partial^{2}}{\partial z 4^{2}} \mathrm{f}(z 1, z 2, z 3, z 4)\right)\right) \mathrm{d}(z \mathscr{D})$

$>\operatorname{valdz2}:=$ factor $($ solve $($ Omega3mod=0, d(z2) ));

vald $z 2:=\left(z 3 v 1\left(\frac{\partial^{2}}{\partial z 4^{2}} \mathrm{~g}\left(z 1, z 2, z 3, z_{4}\right)\right)-2\left(\frac{\partial}{\partial z_{4}} \mathrm{~g}\left(z 1, z 2, z_{3}, z_{4}\right)\right)\right.$

$\left.+z 3\left(\frac{\partial^{2}}{\partial z 4^{2}} \mathrm{f}\left(z 1, z 2, z 3, z_{4}\right)\right)\right) \mathrm{d}(z 1) /($

$\left.\left(\frac{\partial^{2}}{\partial z \mathcal{4}^{2}} \mathrm{f}(z 1, z 2, z 3, z 4)\right)+v 1\left(\frac{\partial^{2}}{\partial z 4_{4}^{2}} \mathrm{~g}(z 1, z \mathscr{2}, z 3, z 4)\right)\right)$

$>\operatorname{valdz3}:=$ factor $(\operatorname{subs}(d(z 2)=v a l d z 2, \operatorname{vvaldz} 3))$; 


$$
\begin{aligned}
& \text { vald } z 3:=\mathrm{d}(z 1)\left(v 1\left(\frac{\partial^{2}}{\partial z 4^{2}} \mathrm{~g}(z 1, z 2, z 3, z 4)\right) \mathrm{g}(z 1, z 2, z 3, z 4)\right. \\
&+\left(\frac{\partial^{2}}{\partial z 4^{2}} \mathrm{f}\left(z 1, z 2, z 3, z_{4}\right)\right) \mathrm{g}(z 1, z 2, z 3, z 4) \\
&\left.-2\left(\frac{\partial}{\partial z 4} \mathrm{f}(z 1, z 2, z 3, z 4)\right) \% 1-2 v 1 \% 1^{2}\right) /( \\
&\left.\left(\frac{\partial^{2}}{\partial z 4^{2}} \mathrm{f}(z 1, z 2, z 3, z 4)\right)+v 1\left(\frac{\partial^{2}}{\partial z 4^{2}} \mathrm{~g}(z 1, z 2, z 3, z 4)\right)\right) \\
& \% 1:=\frac{\partial}{\partial z 4} \mathrm{~g}(z 1, z 2, z 3, z 4)
\end{aligned}
$$

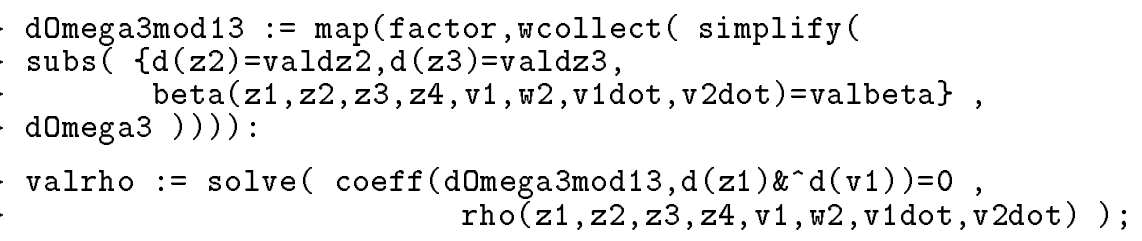

$$
\begin{aligned}
& +\left(\frac{\partial^{2}}{\partial z 4^{2}} \mathrm{~g}(z 1, z 2, z 3, z 4)\right)\left(\frac{\partial}{\partial z 3} \mathrm{~g}(z 1, z 2, z 3, z 4)\right) \mathrm{f}\left(z 1, z 2, z 3, z_{4}\right) \\
& -\left(\frac{\partial^{2}}{\partial z_{4}{ }^{2}} \mathrm{~g}(z 1, z 2, z 3, z 4)\right) \mathrm{g}(z 1, z 2, z 3, z 4)\left(\frac{\partial}{\partial z 3} \mathrm{f}(z 1, z 2, z 3, z 4)\right) \\
& +2 v 1 \% 1^{2}\left(\frac{\partial}{\partial z 3} g(z 1, z 2, z 3, z 4)\right) \\
& +\% 1\left(\frac{\partial^{2}}{\partial z z_{4}^{2}} \mathrm{~g}\left(z 1, z 2, z 3, z_{4}\right)\right) w^{2} \\
& -\left(\frac{\partial^{2}}{\partial z_{4}^{2}} \mathrm{~g}(z 1, z 2, z 3, z 4)\right)\left(\frac{\partial}{\partial z_{4}^{4}} \mathrm{f}\left(z 1, z 2, z 3, z_{4}\right)\right) \mathrm{f}\left(z 1, z 2, z 3, z_{4}\right) \\
& -\left(\frac{\partial^{2}}{\partial z_{4}^{2}} \mathrm{~g}\left(z 1, z 2, z 3, z_{4}\right)\right)\left(\frac{\partial}{\partial z 1} \mathrm{f}\left(z 1, z 2, z 3, z_{4}\right)\right) \\
& +2 \% 1^{2}\left(\frac{\partial}{\partial z 3} \mathrm{f}(z 1, z 2, z 3, z 4)\right) \\
& -z 3\left(\frac{\partial^{2}}{\partial z_{4}^{2}} \mathrm{~g}\left(z 1, z 2, z 3, z_{4}\right)\right)\left(\frac{\partial}{\partial z \mathscr{D}} \mathrm{f}\left(z 1, z 2, z 3, z_{4}\right)\right) \\
& \left.+\left(\frac{\partial^{2}}{\partial z 4^{2}} \mathrm{~g}\left(z 1, z 2, z 3, z_{4}\right)\right)\left(\frac{\partial}{\partial z^{2}} \mathrm{~g}\left(z 1, z 2, z^{3}, z_{4}\right)\right) z_{4}-2 v 1^{2} \% 1^{3}\right)
\end{aligned}
$$

$/ \% 1^{2}$

$\% 1:=\frac{\partial}{\partial z 4} \mathrm{~g}(z 1, z 2, z 3, z 4)$

Let us substitute the value of $\rho$ in $\mathrm{d} \Omega_{3} \operatorname{modulo}\left\{\Omega_{1}, \Omega_{3}\right\}$.

$$
>\text { factor(simplify(subs }
$$




$$
\begin{aligned}
& >\quad \operatorname{rho}(z 1, z 2, z 3, z 4, v 1, w 2, v 1 d o t, v 2 d o t)=\text { valrho, } \\
& >\quad(2 / \mathrm{D} 1) * \text { dOmega3mod13 }))) \text {; } \\
& -\left(\mathrm{d}(z 1) \&^{\wedge} \mathrm{d}(z 4)\right)\left(2 \% 1\left(\frac{\partial^{3}}{\partial z 4^{3}} \mathrm{f}(z 1, z 2, z 3, z 4)\right)\right. \\
& +2 \% 1 v 1\left(\frac{\partial^{3}}{\partial z_{4}^{3}} \mathrm{~g}(z 1, z 2, z 3, z 4)\right)
\end{aligned}
$$

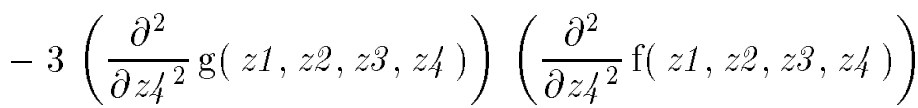

$$
\begin{aligned}
& \left.-3 v 1\left(\frac{\partial^{2}}{\partial z 4^{2}} \mathrm{~g}(z 1, z 2, z 3, z 4)\right)^{2}\right) /(\% 1 \\
& \left.\left(\left(\frac{\partial^{2}}{\partial z 4_{4}^{2}} \mathrm{f}\left(z 1, z 2, z 3, z_{4}\right)\right)+v 1\left(\frac{\partial^{2}}{\partial z_{4}^{2}} \mathrm{~g}\left(z 1, z 2, z 3, z_{4}\right)\right)\right)\right) \\
& \% 1:=\frac{\partial}{\partial z 4} \mathrm{~g}(z 1, z 2, z 3, z 4) \\
& >\mathrm{EE}:=\operatorname{collect}(\text { numer }(\operatorname{get} \operatorname{coeff}(")) \quad, \mathrm{v} 1) \text {; } \\
& E E:=\left(-2 \% 1\left(\frac{\partial^{3}}{\partial z 4^{3}} \mathrm{~g}(z 1, z 2, z 3, z 4)\right)\right. \\
& \left.+3\left(\frac{\partial^{2}}{\partial z 4^{2}} \mathrm{~g}(z 1, z 2, z 3, z 4)\right)^{2}\right) v 1 \\
& -2 \% 1\left(\frac{\partial^{3}}{\partial z_{4}^{3}} \mathrm{f}\left(z 1, z 2, z^{3}, z_{4}\right)\right) \\
& +3\left(\frac{\partial^{2}}{\partial z 4^{2}} \mathrm{~g}(z 1, z 2, z 3, z 4)\right)\left(\frac{\partial^{2}}{\partial z 4_{4}^{2}} \mathrm{f}\left(z 1, z 2, z 3, z_{4}\right)\right) \\
& \% 1:=\frac{\partial}{\partial z 4} \mathrm{~g}(z 1, z 2, z 3, z 4)
\end{aligned}
$$

Both the coefficient of $v_{1}$ and the constant coefficient must be zero, this gives exactly the PDEs (7.52)-(7.53):

$$
\begin{aligned}
& \text { PDE1 }:=-\operatorname{coeff}(E E, \mathrm{v} 1) ; \mathrm{PDE} 2:=-\operatorname{coeff}(\mathrm{EE}, \mathrm{v} 1,0) \text {; } \\
& P D E 1:=2\left(\frac{\partial}{\partial z_{4}} \mathrm{~g}\left(z 1, z 2, z 3, z_{4}\right)\right)\left(\frac{\partial^{3}}{\partial z_{4}^{3}} \mathrm{~g}\left(z 1, z 2, z_{3}, z_{4}\right)\right) \\
& -3\left(\frac{\partial^{2}}{\partial z 4^{2}} g(z 1, z 2, z 3, z 4)\right)^{2} \\
& \text { PDED }:=2\left(\frac{\partial}{\partial z_{4}} \mathrm{~g}\left(z 1, z 2, z_{3}, z_{4}\right)\right)\left(\frac{\partial^{3}}{\partial z_{4}^{3}} \mathrm{f}\left(z 1, z^{2}, z 3, z_{4}\right)\right) \\
& -3\left(\frac{\partial^{2}}{\partial z_{4}^{2}} \mathrm{~g}\left(z 1, z 2, z_{3}, z_{4}\right)\right)\left(\frac{\partial^{2}}{\partial z_{4}^{2}} \mathrm{f}\left(z 1, z 2, z^{3}, z_{4}\right)\right)
\end{aligned}
$$

Hence from lemma $7.9 f$ and $g$ must be given by $f=$ valf and $g=$ valg with:

$$
\begin{aligned}
& >\operatorname{valf}:=(\mathrm{a} 0(z 1, z 2, z 3)+\mathrm{a} 1(\mathrm{z1}, \mathrm{z2}, \mathrm{z3}) * \mathrm{z} 4 \\
& \left.>\quad+a 2(z 1, z 2, z 3) * z 4^{\wedge} 2\right) \\
& /(\operatorname{co}(z 1, z 2, z 3)+c 1(z 1, z 2, z 3) * z 4) ;
\end{aligned}
$$




$$
\begin{aligned}
& \&^{\wedge}(\mathrm{d}(z 1), \mathrm{d}(z \mathscr{2}), \mathrm{d}(z 3))\left(\left(\frac{\partial}{\partial z^{2}} \mathrm{~b} 1(z 1, z \mathscr{2}, z 3)\right) \mathrm{c} 1(z 1, z \mathscr{2}, z 3)\right. \\
& -\mathrm{c} 1(z 1, z 2, z 3) z 3\left(\frac{\partial}{\partial z 2} \mathrm{a} 2(z 1, z 2, z 3)\right) \\
& -\left(\frac{\partial}{\partial z 1} \mathrm{a} 2(z 1, z 2, z 3)\right) \mathrm{c} 1(z 1, z 2, z 3) \\
& +\left(\frac{\partial}{\partial z 3} \mathrm{~b} 1(z 1, z \mathscr{2}, z 3)\right) \mathrm{a} 2(z 1, z \mathscr{2}, z 3) \\
& -\mathrm{b} 1(z 1, z \mathscr{2}, z 3)\left(\frac{\partial}{\partial z 3} \mathrm{a} 2(z 1, z \mathscr{2}, z 3)\right) \\
& +\mathrm{a} 2\left(z 1, z^{2}, z 3\right)\left(\frac{\partial}{\partial z 1} \mathrm{c} 1(z 1, z 2, z 3)\right) \\
& -\mathrm{b} 1(z 1, z 2, z 3)\left(\frac{\partial}{\partial z^{2}} \mathrm{c} 1(z 1, z 2, z 3)\right)-\mathrm{a} 2(z 1, z 2, z 3)^{2} \\
& \left.+z 3\left(\frac{\partial}{\partial z 2} \mathrm{c} 1(z 1, z 2, z 3)\right) \mathrm{a} 2(z 1, z 2, z 3)\right) / \\
& (\mathrm{c} 0(z 1, z 2, z 3)+\mathrm{c} 1(z 1, z 2, z 3) z 4)^{2}
\end{aligned}
$$

Hence this form can be a multiple of $\Omega_{1} \wedge \dot{\Omega}_{3} \wedge \Omega_{3}$ only if it is identically zero. This implies that the following expression must be identically zero:

$>$ EDP3 := numer $(\operatorname{get} \operatorname{coeff}("))$;

$$
\begin{aligned}
& \text { EDP马 }:=\left(\frac{\partial}{\partial z^{2}} \mathrm{~b} 1(z 1, z 2, z 3)\right) \mathrm{c} 1(z 1, z 2, z 3) \\
& -\mathrm{c} 1(z 1, z 2, z 3) z 3\left(\frac{\partial}{\partial z 2} \mathrm{a} 2(z 1, z 2, z 3)\right) \\
& -\left(\frac{\partial}{\partial z 1} \mathrm{a} 2(z 1, z \mathscr{D}, z 3)\right) \mathrm{c} 1(z 1, z 2, z 3) \\
& +\left(\frac{\partial}{\partial z 3} \mathrm{~b} 1(z 1, z 2, z 3)\right) \mathrm{a} 2(z 1, z 2, z 3) \\
& -\mathrm{b} 1(z 1, z 2, z 3)\left(\frac{\partial}{\partial z 3} \mathrm{a} 2(z 1, z \Omega, z 3)\right) \\
& +\mathrm{a} 2(z 1, z \mathscr{D}, z 3)\left(\frac{\partial}{\partial z 1} \mathrm{c} 1(z 1, z \mathscr{Q}, z 3)\right) \\
& -\mathrm{b} 1(z 1, z 2, z 3)\left(\frac{\partial}{\partial z 2} \mathrm{c} 1(z 1, z 2, z 3)\right)-\mathrm{a} 2(z 1, z 2, z 3)^{2} \\
& +z 3\left(\frac{\partial}{\partial z 2} \mathrm{c} 1(z 1, z 2, z 3)\right) \mathrm{a} 2(z 1, z 2, z 3)
\end{aligned}
$$

This is exactly the PDE (4.17) as shown below:

$$
\begin{aligned}
& >\text { Gamma }:=\left(b_{1}(z 1, z 2, z 3)-z 3 * a 2(z 1, z 2, z 3)\right) * d(z 1) \\
& +\mathrm{a} 2(\mathrm{z} 1, \mathrm{z2}, \mathrm{z3}) * \mathrm{~d}(\mathrm{z2})-\mathrm{c} 1(\mathrm{z1}, \mathrm{z2}, \mathrm{z3}) * \mathrm{~d}(\mathrm{z3}) ; \\
& \Gamma:=(\mathrm{b} 1(z 1, z 2, z 3)-z 3 \mathrm{a} 2(z 1, z 2, z 3)) \mathrm{d}(z 1)+\mathrm{a} 2(z 1, z 2, z 3) \mathrm{d}(z 2) \\
& -\mathrm{c} 1(z 1, z 2, z 3) \mathrm{d}(z 3)
\end{aligned}
$$




$$
\begin{aligned}
& >\text { factor(value( d(Gamma) \&- Gamma )); } \\
& \&^{\wedge}\left(\mathrm{d}(z 1), \mathrm{d}\left(z^{2}\right), \mathrm{d}(z 3)\right)\left(\left(\frac{\partial}{\partial z^{2}} \mathrm{~b} 1\left(z 1, z^{2}, z_{3}\right)\right) \mathrm{c} 1(z 1, z 2, z 3)\right. \\
& -\mathrm{c} 1(z 1, z 2, z 3) z 3\left(\frac{\partial}{\partial z 2} \mathrm{a} 2(z 1, z 2, z 3)\right) \\
& -\left(\frac{\partial}{\partial z 1} \mathrm{a} 2(z 1, z \mathcal{L}, z 3)\right) \mathrm{c} 1(z 1, z 2, z 3) \\
& +\left(\frac{\partial}{\partial z 3} \mathrm{~b} 1(z 1, z 2, z 3)\right) \mathrm{a} 2(z 1, z 2, z 3) \\
& -\mathrm{b} 1(z 1, z \mathcal{2}, z 3)\left(\frac{\partial}{\partial z 3} \mathrm{a} 2(z 1, z \mathcal{2}, z 3)\right) \\
& +\mathrm{a} 2(z 1, z 2, z 3)\left(\frac{\partial}{\partial z 1} \mathrm{c} 1(z 1, z 2, z 3)\right) \\
& -\mathrm{b} 1(z 1, z \mathscr{L}, z 3)\left(\frac{\partial}{\partial z \mathscr{L}} \mathrm{c} 1(z 1, z \mathscr{L}, z 3)\right)-\mathrm{a} 2(z 1, z \mathscr{2}, z 3)^{2} \\
& \left.+z 3\left(\frac{\partial}{\partial z^{2}} \mathrm{c} 1(z 1, z \mathscr{2}, z 3)\right) \mathrm{a} 2(z 1, z 2, z 3)\right) \\
& \text { factor ( EDP3 - getcoeff( ") ); } \\
& 0
\end{aligned}
$$

\section{CONCLUSION}

The present paper provides, for the 4-dimensional affine system (1.5), some new necessary and sufficient conditions for existence of linearizing outputs depending on $x$ and $u$. These conditions a re easily computable. They also allow one to treat 3-dimensional non-affine systems. This is very much related to dynamic feedback linearization, or flatness, as explained in section 2, but this paper is however not a general answer to dynamic feedback linearizability of 4-dimensional systems with 2 inputs, for the following three reasons that are subjects for future research to end the study of this small dimension.

One restriction comes from the regularity assumptions. The example presented in section 5 shows that they are not necessary. A thorough treatment of singularities, or at least a clear identification of the real singularities of dynamic feedback linearization is therefore not achieved.

We also restrict our attention to "endogenous feedback". See $[18,11]$ for a discussion of general dynamic feedback and endogenous dynamic feedback. Note that the authors of this latter reference have announced a proof of the fact that general dynamic linearizability would imply linearizability by endogenous dynamic feedback, at least away from some singularities.

We have further restricted the class of dynamic linearization by requiring that the linearizing output depend on $x$ and $u$ only. The natural followup to this work is to decide whether systems which are not $(x, u)$-dynamic Esaim: Cocv, June 1997, Vol. 2, Pr. 151-230 
feedback linearizable are simply not dynamic feedback linearizable (at least endogenously), or if some are $(x, u, \dot{u})$-dynamic linearizable for example ... In fact, no example of a system of these dimensions which admits no pair of linearizing outputs depending on $x$ and $u$ but admits some depending on more time-derivatives of $u$ has ever been exhibited. Since these dimensions are usually these of academic examples - because it is the smallest non-trivial ones - and have been studied a lot, it may seem reasonable to conjecture that the systems that are proved in the present paper to be non $(x, u)$-dynamic linearizable are indeed not linearizable by endogenous $\mathrm{dy}$ namic feedback.

Let us finally make a remark on the method of the proofs. In a sense, the present results amount to giving conditions for some nonlinear partial differential equations to have solutions (see section 2.6). Since the PDEs are high order - see (2.16)-(2.17), and for $(x, u)$-dynamic linearization, the order is higher - one might think that some sophisticated tools for checking integrability, like Spencer cohomology, should be involved. It turns out however that the proofs are all elementary, and never make use of more sophisticated tools than Frobenius theorem. Actually, when using the infinitesimal Brunovsky form and writing the equations for the coefficients of decomposition in elementary transformations of the invertible transformation " $P\left(\frac{\mathrm{d}}{\mathrm{dt}}\right)$ " instead of writing directly the equations for the linearizing outputs, as in the proof of theorem 4.2 or the "alternative" proof of case 6 in theorem 3.1, we use Frobenius theorem to write the equations in a convenient way (like the equation (7.55)-(7.56)-(7.57) for theorem 4.2), but then the arguments used to give conditions for existence of solutions to these equations are in a sense even not first order like Frobenius theorem, but "zeroth order", i.e. the solutions $\alpha, \lambda$ and $b$ in the case (7.55)-(7.56)-(7.57) may be explicitly computed (expression involving functions in the equations of the system) from part of the equations, and the compatibility conditions are obtained by substituting these expressions in the remaining equations. It is of course tempting to ask whether in general when using the infinitesimal Brunovsky form to test for existence of linearizing outputs depending on a pre-defined number of timederivatives of the inputs, this feature always appears - the equations for the coefficients of the invertible transformation contain enough non-differential equations to obtain them solving non-differential equations- or if this is particular to the small dimensions considered here.

\section{APPENDIX: SOME FACTS ON PFAFFIAN SYSTEMS}

In this section, we recall some very basic definitions on Pfaffian systems, and some precise facts we are going to use. For details or proofs, see e.g. [25] or $[6]$.

A Pfaffian system I of rank $r$ around a point can be defined as a module (over smooth functions) of differential 1-forms which is generated by $r$ 1-forms which are point-wisely linearly independent around this point, or also as an ideal of differential forms (of arbitrary degrees, with the exterior product as "multiplication"), which has the peculiarity of being generated by independent 1 -forms. It is defined by giving $r$ independent 1 -forms. $r$ 1-forms which generate the same module define the same Pfaffian system. 
A congruence like $\Omega_{1} \equiv \Omega_{2}$ modulo $\left\{\eta_{1}, \eta_{2}, \ldots\right\}$ where the $\Omega_{i}$ 's are 2forms and the $\eta_{j}$ 's are 1 -forms (we only need this) means modulo the ideal generated by $\left\{\eta_{1}, \eta_{2}, \ldots\right\}$, i.e. it means that there exists some forms $\alpha_{j}$ such that $\Omega_{1}-\Omega_{2}=\eta_{1} \wedge \alpha_{1}+\eta_{2} \wedge \alpha_{2}+\ldots$; it is equivalent to $\left(\Omega_{1}-\Omega_{2}\right) \wedge \eta_{1} \wedge$ $\eta_{2} \wedge \ldots=0$.

A Pfaffian system also defines an "orthogonal distribution", spanned by the vector fields which annihilate these 1-forms.

We will only be interested in the case $m=1$ or $m=2$, and we therefore speak of the Pfaffian system $I=\{\omega\}$ or $I=\left\{\omega_{1}, \omega_{2}\right\}$.

It is completely integrable if it is, locally, generated by 1 (resp 2) exact 1-forms, or equivalently, by Frobenius theorem, if $\mathrm{d} \omega \equiv 0 \bmod \omega \operatorname{lo}\{\omega\}$ (resp. $\mathrm{d} \omega_{i} \equiv 0$ modulo $\left\{\omega_{1}, \omega_{2}\right\}$ for $\left.i=1,2\right)$, or also if the orthogonal distribution being closed under Lie brackets. We call first integral of the Pfaffian system, or of the orthogonal distribution a function $h$ such that $\mathrm{d} h \neq 0$ and $\mathrm{d} h \in I$.

Derived System. For a given Pfaffian system $I$, consider the module made of the forms of degree 1 which are in $I$ and whose exterior derivative (form of degree 2) is also in $I$; at points where it has constant rank, this module defines a Pfaffian system called the derived system $I^{(1)}$ of $I$. Iterating this process, one ends either with the zero Pfaffian system or with an integrable one. A Pfaffian system is equal to its first derived system if and only if it is integrable. In the case of a Pfaffian system of rank 1 , either it is integrable or its derived system is zero; in the case of a Pfaffian system of rank $2\left\{\omega_{1}, \omega_{2}\right\}$, either it is integrable, or there exists (non both zero) functions $\lambda_{1}$ and $\lambda_{2}$ such that

$$
\lambda_{1} \mathrm{~d} \omega_{1}+\lambda_{2} \mathrm{~d} \omega_{2} \equiv 0 \operatorname{modulo}\left\{\omega_{1}, \omega_{2}\right\},
$$

and in this case the first derived system is $\left\{\lambda_{1} \omega_{1}+\lambda_{2} \omega_{2}\right\}$ or there exists no such functions (i.e. the restrictions of $d \omega_{1}$ and $d \omega_{2}$ to the annihilator of $\left\{\omega_{1}, \omega_{2}\right\}$ are two linearly independent bilinear forms), and then the derived system is zero. The orthogonal distribution to the derived system of a given Pfaffian system is spanned by the orthogonal distribution to this system plus all the Lie brackets between two vector fields in this distribution:

$$
\left(I^{(1)}\right)^{\perp}=I^{\perp}+\left[I^{\perp}, I^{\perp}\right] .
$$

Cartan Characteristic System. The Cartan characteristic system $\mathcal{C}(I)$ of a given Pfaffian system $I$ may be defined through the vectors that it annihilates:

$$
\mathcal{C}(I)^{\perp}=\left\{X \in I^{\perp} /\left[X, I^{\perp}\right] \subset I^{\perp}\right\} .
$$

It is always integrable if it has constant rank, and a Pfaffian system is integrable if and only if it is equal to its Cartan characteristic system.

There is a basis of the Pfaffian system whose elements are linear combination of some $\mathrm{d} \psi_{i}$, with coefficients functions of the on $\psi_{i}$ 's only, where the $\psi_{i}$ 's are all first integrals of $\mathcal{C}(I)$, and $\mathcal{C}(I)$ is the smallest Pfaffian system having this property.

For a non-integrable system of rank $1\{\omega\}$, it is always possible, where the rank of the characteristic system is constant, to find $2 p$ independent 1-forms Esaim: Cocv, June 1997, Vol. 2, Pr. 151-230 
$\eta_{i}$ such that the rank of $\left\{\omega, \eta_{1}, \ldots, \eta_{2 p}\right\}$ is $2 p+1$ and

$$
\mathrm{d} \omega \equiv \eta_{1} \wedge \eta_{2}+\eta_{3} \wedge \eta_{4}+\ldots+\eta_{2 p-1} \wedge \eta_{2 p} \operatorname{modulo}\{\omega\}
$$

and the characteristic system is then $\left\{\omega, \eta_{1}, \ldots, \eta_{2 p}\right\}$ (and this is automatically completely integrable).

For a non-integrable system of rank $2\left\{\omega_{1}, \omega_{2}\right\}$, all we need is the following: if it is possible to express this Pfaffian system with 4 variables $\chi_{1}, \chi_{2}, \chi_{3}, \chi_{4}$ (i.e. there exists a basis of this Pfaffian system made of two 1-forms which are linear combinations of $\mathrm{d} \chi_{1}, \mathrm{~d} \chi_{2}, \mathrm{~d} \chi_{3}, \mathrm{~d} \chi_{4}$ with coefficients functions of $\chi_{1}, \chi_{2}, \chi_{3}, \chi_{4}$ only), then its characteristic system is $\left\{\mathrm{d} \chi_{1}, \mathrm{~d} \chi_{2}, \mathrm{~d} \chi_{3}, \mathrm{~d} \chi_{4}\right\}$, and for any forms $\eta_{1}$ and $\eta_{2}$ such that $\left\{\omega_{1}, \omega_{2}, \eta_{1}, \eta_{2}\right\}$ spans the same module as $\left\{\mathrm{d} \chi_{1}, \mathrm{~d} \chi_{2}, \mathrm{~d} \chi_{3}, \mathrm{~d} \chi_{4}\right\}$, we have

$$
\mathrm{d} \omega_{k}=\omega_{1} \wedge \Gamma_{k, 1}+\omega_{2} \wedge \Gamma_{k, 2}+\lambda_{k} \eta_{1} \wedge \eta_{2}
$$

for some 1 -forms $\Gamma_{k, j}$ and some functions $\lambda_{k}$.

The author wishes to acknowledge Claude Moog and Eduardo Aranda from Laboratoire d'Automatique de Nantes, France; the present work started essentially as an application of previous common work with them.

He also wishes to thank Pierre Rouchon, from Ecole des Mines, Paris, for communicating the preprint [22] and for discussions on the necessary condition for non-affine systems used in section 6 .

\section{REFERENCES}

[1] E. Aranda-Bricaire, C.H. Moog, J.-B. Pomet: An infinitesimal brunovsky form for nonlinear systems with applications to dynamic linearization, in Geometry in Nonlinear Control and Differential Inclusions, B. Jakuczyk, W. Respondek, and T. Rzezuchowski, eds., Banach Center Publications, 32, Warsaw, Poland, 1995, Institute of Mathematics, Polish Academy of Sciences, 19-33.

[2] E. Aranda-Bricaire, C.H. Moog, J.-B. Pomet: A linear algebraic framework for dynamic feedback linearization, IEEE Trans. Automat. Control, 40, 1995, 127-132.

[3] B. Bonnard: Feedback equivalence for nonlinear systems and the time-optimal control problem, SIAM J. on Control and Optim., 29, 1991, 1300-1321.

[4] R.W. Brockett: Feedback invariants for nonlinear systems, in IFAC World Congress, Helsinki, 1978, Birkäuser.

[5] P. Brunovský: A classification of linear controllable systems, Kybernetica, 6, 1970, $176-188$.

[6] R.L. Bryant, S.S. Chern, R.B. Gardner, H.L. Goldschmitt, P.A. Griffiths: Exterior Differential Systems, Mathematical Sciences Research Institute Publications 18, Springer-Verlag, 1991.

[7] B. Charlet, J. Lévine, R. Marino: Sufficient conditions for dynamic state feedback linearization, SIAM J. on Control and Optim., 29, 1991, 38-57.

[8] M. Fliess, J. Lévine, P. Martin, P. Rouchon: On differentially flat nonlinear systems, in Nonlinear Control Systems Design, M. Fliess, ed., Pergamon Press, 1992, 408-412.

[9] M. Fliess, J. Lévine, P. Martin, P. Rouchon: Linéarisation par bouclage dynamique et transformations de Lie-Bäcklund, C. R. Acad. Sci., Paris, Série I, 317, 1993, 981-986.

[10] M. Fliess, J. Lévine, P. Martin, P. Rouchon: Towards a new differential geometric setting in nonlinear control, in Proc. International Geometrical Coll., Moscow, 1993.

[11] M. Fliess, J. Lévine, P. Martin, P. Rouchon: Flatness and defect of nonlinear systems: Introductory theory and examples, Int. J. Control, 61, 1995, 1327-1361.

[12] L.R. Hunt, R. Su, G. Meyer: Design for multi-input nonlinear systems, in Differential Geometric Control Theory, R. Brockett, ed., Birkhäuser, 1983, 258-298. 
[13] B. Jakubczyk: Equivalence and invariants of nonlinear control systems, in Nonlinear Controllability and Optimal Control, H.J. Sussmann, ed., Marcel Dekker, New-York, 1990.

[14] B. Jakubczyk: Dynamic feedback equivalence of nonlinear control systems. Preprint, 1994.

[15] B. Jakubczyk, W. Respondek: On linearization of control systems, Bull. Acad. Polonaise Sci. Ser. Sci. Math., 28, 1980, 517-522.

[16] C. Leleu, J.-B. Pomet: A package in "maple" to decide on $(x, u)$-dynamic linearizability of control systems with four states and two inputs., rapport de recherche, INRIA, 1997, to appear. (URL: http://www.inria.fr/publications-eng.html).

[17] J. Lévine, R. Marino: On dynamic feedback linearization on $\mathbb{R}$, in 29th IEEE Conf. on Decision \&\& Control, 1990, 2088-2090.

[18] P. Martin: Contribution à l'étude des systèmes différentiellement plats, $\mathrm{PhD}$ thesis, L'École Nationale Supérieure des Mines de Paris, 1992.

[19] J.-B. Pomet: A differential geometric setting for dynamic equivalence and dynamic linearization, in Geometry in Nonlinear Control and Differential Inclusions, B. Jakuczyk, W. Respondek, T. Rzezuchowski, eds., Banach Center Publications 32, Warsaw, Poland, 1995, Institute of Mathematics, Polish Academy of Sciences, 319-339.

[20] J.-B. Pomet, C.H. Moog, E. Aranda-Bricaire: A non-exact Brunovsky form and dynamic feedback linearization, in 31 st IEEE Conf. on Decision and Control, Tucson, U.S.A., Dec. 1992, 2012-2017.

[21] J.-F. Pommaret: Partial Differential Equations and Group Theory, Mathematics and its Applications 293, Kluwer Academic Publishers, Dordrecht, Boston, London, 1994.

[22] P. Rouchon: Necessary condition and genericity of dynamic feedback linearization, Journal of Mathematical Systems, Estimation, and Control, 4, 1994, 1-14.

[23] W. Shadwick: Absolute equivalence and dynamic feedback linearization, Systems \& 8 Control Lett., 15, 1990, 35-39.

[24] W. Sluis: A necessary condition for dynamic feedback linearization, Systems \& Control Lett., 21, 1993, 277-283.

[25] S. Sternberg: Lectures on Differential Geometry, Chelsea Publishing Co., New York, 2nd ed., 1983. 